\title{
Physicochemical Characterization of Passive Films and Corrosion Layers by Differential Admittance and Photocurrent Spectroscopy
}

\author{
Francesco Di Quarto, Fabio La Mantia, and \\ Monica Santamaria \\ Dipartimento di Ingegneria Chimica dei Processi e dei Materiali, Universita' degli \\ studi di Palermo, Viale delle Scienze, 90128 Palermo
}

\section{INTRODUCTION}

The stabilization of metallic surfaces against corrosion processes in natural and industrial environment rests on the onset of passivity condition with a subsequent drastic reduction of the corrosion rate of the underlying metallic substrate. In spite of a longstanding controversy it is now universally accepted that a passive metal is usually covered by a thin or thick external layer the whose physicochemical properties control the evolution of corrosion process as well as the possible reactions occurring at metal/oxide and oxide/electrolyte interfaces. ${ }^{1,2}$ In many cases of practical importance, passivity of metals is reached in presence of very thin (few $\mathrm{nm}$ thick) layer which makes the complete physico-chemical characterization a very complex task requiring the use of different

Modern Aspects of Electrochemistry, Number 46, edited by S. -I. Pyun and J. -W. Lee, Springer, New York, 2009. 
powerful in situ and/or ex situ techniques. This is particularly true if we want to get information pertaining to the chemical composition, morphology, crystalline or disordered nature and solid-state properties of the passive layers.

Many useful information on the film composition can be gathered from ex situ techniques (Auger, ESCA, XPS, SIMS, RBS, GDOES) although they suffer some limitations and drawback specially when investigating very thin films, owing to the risk of changing structure and composition of the passive film on going from the potentiostatic control in solution to the vacuum. According to this a large agreement exists on the advantages of passive film characterization based on in situ techniques or controlled transfer under inert atmosphere on going from electrochemical cell to analytical equipment. ${ }^{3-11}$

Besides more traditional (and mainly optical) techniques (differential and potential modulated reflectance, ellipsometry, interferometry, Fourier transform infrared spectroscopy, Raman and Mössbauer spectroscopy), new in situ techniques have been introduced in the last years, which are capable of providing useful information on structure, composition, morphology and thickness of passive films, such as: EXAFS, XANES, EQCM, STM and AFM. The use of these analytical techniques has improved our understanding of the structure and composition of passive films grown on metal and alloys. ${ }^{1-14}$

As for the study of solid state and electronic properties of passive films and corrosion layers differential admittance (DA) and photocurrent spectroscopy (PCS) techniques have been largely used by electrochemists and corrosion scientists during the years. Both techniques take advantage from the pioneering works of Garrett and Brattain in the mid 1950s and from the advent of Gerischer's theory and its systematic use for interpreting the kinetics of electron and ion transfer reactions (ETR and ITR) at the semiconductor/electrolyte interface. ${ }^{15-25}$ We have to mention that both ITR and ETR are involved in determining the kinetics of growth and the breakdown processes of passive films. ${ }^{1,226-33}$ In many cases the combined use of both DA and PCS techniques is able to provide the information necessary to locate the characteristic energy level (flat band potential, $U_{f b}$, conduction and valence band edge $E_{C}, E_{V}$ ) of the passive film/electrolyte junction. 
The location of characteristics energy levels of the metal/passive film and passive film/electrolyte junctions is a preliminary task for understanding the kinetics of ion and electron transfer reactions at passive film electrolyte-interface. In fact, both the ITR and ETR are controlled by the:

- $\quad$ energetics of the metal/film and film/electrolyte interfaces

- electronic properties of the passive film.

In the case of differential admittance technique apart the seminal papers of Mott $^{34}$ and Schottky ${ }^{35}$ on the rectifying properties of metal/semiconductor contacts (copper/cuprous oxide) there is no doubt that the classical papers of Dewald on the $\mathrm{ZnO} /$ electrolyte interface, ${ }^{36}$ showing the validity of Mott-Schottky analysis for the location of characteristic levels of the semiconductor/electrolyte junction, opened up a new route for a deeper understanding of the structure of SC/electrolyte interface.

If we take into account that in the case of passive films the extreme thinness and their disordered or amorphous structure adds further complications to the possible interpretative models of DA data, it is not surprising that in spite of the numerous studies on passive film/electrolyte interfaces an unambiguous picture or generalized acceptance of interpretative models is still lacking.

Together with differential admittance studies and among other optical methods, PCS has gained a large consideration in the last decades as in situ technique for the characterization of photoconductive passive film able to provide information not only on the location of characteristic energy level of passive film/electrolyte junction and internal photoemission threshold $\left(E_{t h}\right)$ at the metal/passive film interface, but also as a possible analytical tool for identifying the nature of passive film and corrosion layers. ${ }^{37-40}$ The attractive features of PCS are due to the fact that it is a nondestructive technique based on the analysis of the electrochemical response (photocurrent or photopotential) of the passive electrode/electrolyte interface under irradiation with photons of suitable energy and intensity. Often the choice of a potentiostatic control is preferred, by taking into account the prominent role of the electrode potential in the establishment of electrochemical equilibrium involving different metal oxidation states and reactivity of the passive films. ${ }^{41}$ 
It is in the aims of this work to stress the limitations of a traditional approach, based on the theory of ideal crystalline semiconductor, to the study of semiconducting behavior of passive films and to provide, possibly, a more general interpretative frame for a deeper understanding of the semiconducting properties of passive films. At this aim after an initial introduction to the theory of MottSchottky barrier valid for ideal single crystal SC/electrolyte junction a more extended introduction to the theory of amorphous semiconductor Schottky barrier will be presented. In this frame some inconsistencies usually encountered in literature of thin passive films will be discussed and possible alternative explanations suggested in agreement with the more realistic model of the solid state properties in disordered and amorphous materials.

In the second part of the work a short introduction to the optical properties of crystalline semiconductor/electrolyte interface will be presented as preliminary to a more extended discussion on the influence of lattice disorder on the optical properties of semiconductor and insulating materials. New features related to the extremely thin thickness of passive films will be presented and discussed. Finally the use of PCS as an analytical tool for identifying the possible nature of corrosion and passive film will be discussed on the basis of a semi-empirical correlation between optical band gap of oxides and composition of passive films grown on metal and alloys.

\section{IMPEDANCE MEASUREMETS FOR PASSIVE FILMS}

\section{Semiconductor/Electrolyte Interface}

With very few exceptions, most of passive layers grown on metals and alloys behave like semiconducting or insulating materials. Accordingly, widespread use of the theory of crystalline semiconductors has been made to discuss the behavior of passive film/electrolyte junction. Such an extension is not always critically checked by taking into account whether the hypothesis underlying the theory of bulk crystalline materials are also valid in the case of the thin passive films investigated. Moreover, in spite of the exist- 


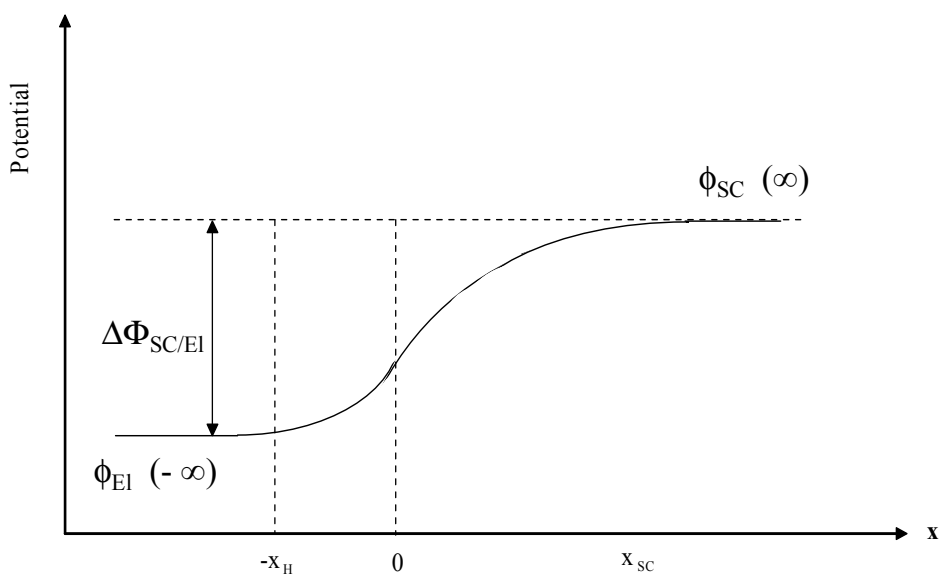

Figure 1. Galvani potential distribution across an n-type SC/El interface in absence of specific adsorption of ions in solution and surface states.

ing evidence that in many cases the passive films are amorphous or strongly disordered such an information is not usually taken into account to explain the admittance behavior of the junction neither to modify the traditional approach to the study of the interface by using the more pertinent theory of amorphous semiconductor Schottky barrier. For a better understanding of the influence of amorphous nature on the differential admittance and photocurrent spectroscopy measurements a brief introduction on the structure of semiconductor/electrolyte interface will be given to derive some equations usually employed to investigate the structure of such interface.

With respect to the metal/electrolyte interface in the case of $\mathrm{SC} /$ electrolyte interface the main differences stem out from the different electronic structure of the electrode. ${ }^{16-25}$ In fact, differently than in a metal, owing to a much lower density of free carriers in the conduction band of semiconductor a much larger screening length in SC electrode is necessary to neutralize the excess of charge lying in the solution side.

In Fig. 1 we report the schematic Galvani potential distribution across an n-type $\mathrm{SC} / \mathrm{El}$ interface in absence of specific absorption of ions in solution. According to Fig. 1 the total potential drop across the $\mathrm{SC} / \mathrm{El}$ interface is given by: 


$$
\Delta \Phi_{S C / E l}=\left\{\phi_{S C}(\infty)-\phi_{S C}(0)\right\}+\left\{\phi_{S C}(0)-\phi_{E l}(-\infty)\right\}
$$

where the first term in the bracket of Eq. (1) represents the Galvani potential drop from the bulk (zero electric field) to the surface of $\mathrm{SC} / \mathrm{El}$ junction, whilst the term in the second bracket is the Galvani potential drop occurring into the compact and diffuse double layer (if any) of the electrolytic solution. In presence of concentrated electrolyte the diffuse double layer is missing and we can rearrange the previous equation as:

$$
\Delta \Phi_{S C / E l}=\Delta \Phi_{S C}+\Delta \Phi_{H}
$$

where $\Delta \Phi_{S C}$ and $\Delta \Phi_{H}$ represent the potential drop into SC and the Helmholtz double layer respectively. In equilibrium conditions the potential drop inside the semiconductor can be calculated by solving the Poisson equation (see below) under the same conditions used for an ideal semiconductor/metal Schottky barrier and by taking into account that the potential drop within the semiconductor is only a part of the total potential difference measured with respect to a reference electrode. Moreover, by assuming that in presence of a sufficiently concentrated $(>0.1 \mathrm{M})$ electrolytic solution the potential drop in the diffuse double layer is negligible, the measured differential capacitance of the interface, $C_{m}$, in electrochemical equilibrium can be defined as: ${ }^{36}$

$$
C_{m}=\left|\frac{\partial Q_{S C}}{\partial \Delta \Phi_{S C / E l}}\right|=\left|\frac{\partial Q_{S C}}{\partial \Delta \Phi_{S C}} \frac{\partial \Delta \Phi_{S C}}{\partial \Delta \Phi_{S C / E l}}\right|=C_{S C}\left(1-\frac{C_{m}}{C_{H}}\right)
$$

where $C_{S C}$ is the capacitance of the space charge region in the semiconductor, and $C_{H}$ is the capacitance of the Helmholtz compact double layer. In terms of equivalent electrical circuit the interface can be represented by two capacitors in series:

$$
\frac{1}{C_{m}}=\frac{1}{C_{S C}}+\frac{1}{C_{H}}
$$


The value of $C_{S C}$ changes with the width of space charge region within the semiconductor, $X_{S C}$, and it is a function of the potential drop, $\Delta \Phi_{\mathrm{SC}}$, within the SC whilst a constant value (10-30 $\mu \mathrm{F} \mathrm{cm}{ }^{-2}$ ) is usually assumed for $C_{H}$.

The region of SC necessary for screening the potential drop $\Delta \Phi_{\mathrm{SC}}$ defines the space charge region. The width of the spacecharge region in crystalline semiconductor (c-SC) changes with the potential drop according to the following equation:

$$
X_{S C}=X_{S C}^{0}\left(\left|\Delta \Phi_{S C}\right|-\frac{k T}{e}\right)^{0.5}
$$

where $X_{S C}^{0}$ represents the space-charge width into the SC electrode at $1 \mathrm{~V}$ of band bending $\left(\Delta \Phi_{\mathrm{SC}}=\psi_{\mathrm{SC}}=1 \mathrm{~V}\right)$ and its value depends on the concentration of mobile carriers into the SC. In the hypothesis of completely ionized donors (n-type SC) or acceptor (ptype SC) the expression for such a characteristics length is given by:

$$
X_{S C}^{0}=\left(\frac{2 \varepsilon_{o x} \varepsilon_{0}}{e N_{D / A}}\right)^{0.5}
$$

where $N_{D / A}$ are the donor (or acceptor) concentration in $\mathrm{cm}^{-3}, \varepsilon_{\mathrm{ox}}$ and $\varepsilon_{0}$ are the $\mathrm{SC}$ dielectric constant and the vacuum permittivity, respectively. By assuming for the passive film dielectric constant the typical value of $\sim 10$ and a donor concentration of $10^{19} \mathrm{~cm}^{-3}$ we get for $X_{S C}^{0}$ a value around $100 \AA \mathrm{V}^{-0.5}$.

In Figs. $2 a$ and $2 b$ we report the schematic diagram of an $n-$ type SC/electrolyte junction in energy-distance coordinates at flat band conditions $\left(X_{S C}=0\right)$ and under slight depletion $\left(X_{S C}>0\right)$. $\Delta \Phi_{\mathrm{SC}}=0$ (no potential drop within the $\mathrm{SC}$ ) corresponds to the special flat band condition reported in Fig. 2a whilst an anodic $\Delta \Phi_{\mathrm{SC}}>$ 0 (n-type SC) polarization corresponds to the conditions depicted in Fig. 2b. In the case of p-type material a space charge layer develops inside the $\mathrm{SC}$ for $\Delta \Phi_{\mathrm{Sc}}<0$. 


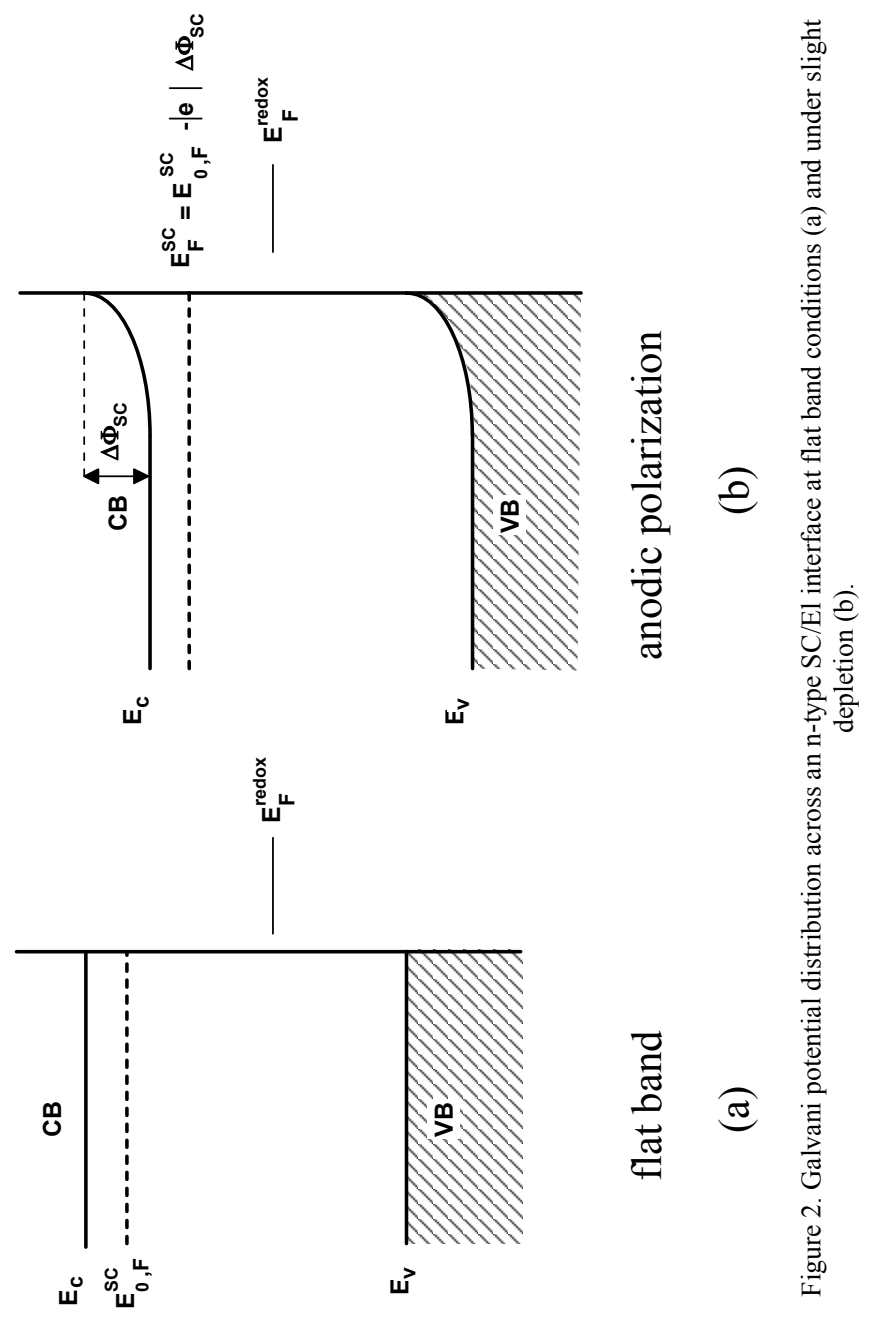


In terms of electrode potential, $U_{E}$, for not heavily doped SC and in absence of an appreciable density of electronic surface states (SS), we can write:

$$
\Delta \Phi_{S C}=U_{E}-U_{f b}(r e f)
$$

where $U_{f b}(r e f)$ represents the flat band potential measured with respect to a reference electrode in the electrochemical scale.

\section{Location of Characteristic Energy Level in c-Semiconductor/Electrolyte Interface}

The first task in the location of the energetics of a semiconductor/ electrolyte interface is to derive the flat band potential of the junction, corresponding to the condition of zero Galvani potential drop inside the SC. A common practice in electrochemistry is to get such a parameter by measuring the differential capacitance of the junction defined as above:

$$
\frac{1}{C_{m}} \cong \frac{1}{C_{S C}}
$$

by assuming $1 / C_{H}<<1 / C_{s c}$. In order to get the expression of the differential capacitance $\mathrm{C}_{\mathrm{SC}}$ it is first necessary to solve the Poisson-Boltzmann equation for the band-bending in the $\mathrm{SC}$ and then calculate the charges from Gauss's law. ${ }^{36}$ and refs therein Under the simplifying assumptions of:

- crystalline semiconductor electrode homogeneously doped under depletion regime;

- fully ionized single donor (or acceptor, for p-type SC) level;

- absence of deep lying donor (acceptor) levels in the forbidden gap of the SC;

- negligible contribution of surface states and minority carriers to the measured capacitance;

- absence of Faradaic processes at the SC/El interface;

- width of space-charge layer much lower than the semiconductor thickness; 
it is possible to get a simplified expression for the space charge capacitance, which coincides with the well-known Mott-Schottky equation:

$$
\left(\frac{1}{C_{S C}}\right)^{2}=\left(\frac{2}{\varepsilon_{o x} \varepsilon_{0}|e| N_{D}}\right)\left(\Delta \Phi_{S C}-\frac{k T}{|e|}\right)
$$

where $\Delta \Phi_{\mathrm{SC}}=\left(U_{E}-U_{f b}\right)$ and $N_{D}$ is the density of ionized donors in the n-type SC. The previous equation holds for a p-type material, with the minus sign in front of $\Delta \Phi_{\mathrm{sc}}$ and $N_{A}$ instead of $N_{D}$. The extrapolation to zero of the first term in M-S equation provides the value of the intercept with voltage axis $U_{0}$, whilst from the slope of Eq. (8) it is possible to derive the concentration of donor (or acceptor) in the SC. A more general equation taking into account the degree of dissociation of donors (acceptors) has been reported ${ }^{36}$ but rarely employed in the study of passive film. ${ }^{28}$

The assumption of neglecting the contribution of Helmholtz capacitance to $C_{m}$ can seriously affect the value of flat band potential. In fact we recall that the following relationship exists between the intersection of the M-S plot with potential axis, $\mathrm{U}_{0}$, and the flat potential value: ${ }^{42-44}$

$$
\begin{array}{cc}
U_{f b}=U_{0}-\frac{k T}{e}+\frac{\varepsilon_{o x} \varepsilon_{0} e N_{D}}{2 C_{H}^{2}} & \text { for n-type SC } \\
U_{f b}=U_{0}+\frac{k T}{e}-\frac{\varepsilon_{o x} \varepsilon_{0} e N_{A}}{2 C_{H}^{2}} & \text { for n-type SC }
\end{array}
$$

The required correction in the $U_{f b}$ value remains negligible as long as the dielectric constant value and donor density are small but it becomes important as $\varepsilon_{\mathrm{ox}}$ or $N_{D}$ (or both) increase close to values as those sometimes reported for passive films (see below). The location of the Fermi level of the c-SC, in the electrochemical scale, is carried out by means of the equation: ${ }^{39}$

$$
E_{f}^{0}(E l)=-|e| U_{f b}(r e f)
$$


According to the theory at any other electrode potential the Fermi level of the SC, in the hypothesis of electronic equilibrium trough the space charge region, will be located by assuming $E_{f}=E_{f}^{0}-$ $|e| \Delta \Phi_{\mathrm{sc}}$.

The location of the remaining energy levels of the junction is performed by means of the usual relationships for $n$ - and p-type semiconductors:

$$
\begin{array}{ll}
E_{C}=E_{F}^{0}+k T \ln \left(\frac{N_{C}}{N_{D}}\right) & \text { for n-type SC } \\
E_{V}=E_{F}^{0}-k T \ln \left(\frac{N_{V}}{N_{A}}\right) & \text { for p-type SC } \\
E_{g}=E_{C}-E_{V} &
\end{array}
$$

where $N_{C}$ (and $N_{V}$ ) is the effective density of states (DOS) at the bottom (top) of the SC conduction (valence) band, $E_{C}$ and $E_{V}$ the conduction and valence band edges, respectively, and $E_{g}$ is the band gap of the SC. The simplified method reported above and based on the use M-S theory for locating the energy levels at the $\mathrm{SC} / \mathrm{El}$ interface is very popular among the corrosion scientists. However, we have to mention that, although the validity of Eq. (8) has been tested rigorously for several SC/El interfaces, ${ }^{43-50}$ since the seminal work of Dewald ${ }^{36}$ on single crystal $\mathrm{ZnO}$ electrodes, in many cases a misuse has been made of such equation.

In order to highlight this aspect we come back to the limitations included in the use of the above reported equations. As for the effective DOS at conduction (valence) band edge for n-type ( $p$ type) $\mathrm{SC}$ it is given by:

$$
N_{C}=2 M_{C}\left(\frac{2 \pi m^{*} k T}{h^{2}}\right)^{3 / 2}
$$

where $M_{C}$ is the number of equivalent minima (maxima) in the conduction (valence) band, $m^{*}$ is the effective mass for the DOS in the corresponding band and the other symbols have their usual meaning. By assuming $M_{C}=1$ and $m^{*}$ equal to the mass of free electron, $\mathrm{m}_{0}$, we get $N_{C}=2.5 \times 10^{19} \mathrm{~cm}^{-3}$ at room temperature. By 
taking into account that Eq. (11) on the hypothesis that the following relationship:

$$
\left(E_{v}+3 k T\right) \leq E_{F} \leq\left(E_{c}-3 k T\right)
$$

holds, we can estimate for an hypothetical donor (acceptor) density equal to $10^{19} \mathrm{~cm}^{-3}$ an effective mass of electron (holes) in the conduction (valence) band $m^{*} \geq 7$. Such a value of $m^{*}$ is not unusual for transition metal oxides so that we can also estimate the corresponding space charge thickness of the semiconducting oxide by means of Eq. (6) reported above. According to the previous calculations and by assuming $\Delta \Phi_{\mathrm{SC}}=0.5 \mathrm{~V}$ a value of space charge length of $\sim 9 \mathrm{~nm}$ for iron oxide $\left(\varepsilon_{\mathrm{ox}}=15\right)$ and $\sim 8 \mathrm{~nm}$ for nickel oxide $\left(\varepsilon_{\mathrm{ox}}=12\right)$ are estimated. Both these values are larger than the oxide film thickness usually reported for passive iron $(3 \sim 5 \mathrm{~nm})$, nickel $(2 \sim 3 \mathrm{~nm})$, chromium and stainless steels $(2 \sim 3 \mathrm{~nm})$ in different solutions before the onset of the transpassive region, ${ }^{51-56}$ so that the hypothesis of SC space charge width much lesser than the thickness of SC could be untenable and the electrical equivalent circuit of the junction should be modified by adding a further capacitance in parallel with the $\mathrm{C}_{\mathrm{SC}}$ term which accounts for the metal contribution to the total stored charge. ${ }^{57}$

On the other hand donor or acceptor concentration larger than $10^{20} \sim 10^{21} \mathrm{~cm}^{-3}$ for passive films on iron, nickel and stainless steel have been reported ${ }^{58-66}$ and up to $10^{22} \mathrm{~cm}^{-3}$ in some chromium carbon steel. ${ }^{67}$ At such high level of donor or acceptor concentration the applicability of classical M-S theory to the study of a degenerate (or strong impurity metal ${ }^{68}$ ) semiconductor/electrolyte interface is open to serious doubts. By considering that in this case the Fermi level should be located well above (below) the conduction (valence) band edge, the passive film/electrolyte interface becomes now more similar to a semimetal/electrolyte interface for which a different theoretical approach has been suggested. ${ }^{31,69-70}$

In presence of such large density of donor (acceptor) concentration the experimental data should be taken with caution and the electrical equivalent circuit, employed to extract the space-charge capacitance data, carefully scrutinized in order to verify if the restrictions underlying the simple M-S analysis are satisfied specially in absence of ideally polarizable interface. Moreover at such high donor (acceptor) concentration, neglecting the contribution of 
the capacitance of the Helmholtz double layer to the measured capacitance is no more acceptable whilst the assumption of a potential independent $\mathrm{C}_{\mathrm{H}}$ value, at negative potentials with respect to the flat band condition, should be tested. ${ }^{31}$ For highly doped materials, as previously mentioned, the values of $U_{f b}$ can differ considerably from the intercept value $U_{0}$. By substituting the value of dielectric constant usually reported for passive iron $\left(\varepsilon_{\mathrm{ox}}=15\right)$ and an average $N_{D}$ value of $10^{21} \mathrm{~cm}^{-3}$ we obtain (see Eqs. 9a,b) a difference between the flat band potential and the intersection voltage $U_{0}$ ranging from $0.26 \mathrm{~V}$ up to $1.06 \mathrm{~V}$ by assuming for $C_{H}$ values ranging between 20 and $10 \mu \mathrm{F} \mathrm{cm}^{-2}$.

Further limitations in the application of classical M-S analysis to passive films come out from the strong frequency dependence usually observed in the differential capacitance values of the junctions affecting considerably both the slope $\left(N_{D}, N_{A}\right)$ and the flat band potential values. As possible explanations of such a behavior the presence of deep lying donor (acceptor) level and/or the presence of surface states has been frequently invoked in the case of crystalline semiconductors and semiconducting passive film. However, the apparent measured density of donor (acceptor) carried out trough the M-S equation is meaningless whilst the location of characteristics energy levels of the junction performed by means of Eqs. (11) may be misleading as discussed in the specialized literature several years ago. ${ }^{71,72}$ In the case of c-SC the possible physical cause of such frequency dependence has been also attributed to the presence of an external (disordered or amorphous) layer, which after chemical etching of the surface of c-SC could be removed to restore the expected behavior. ${ }^{73}$

In the case of thin passive film the presence of a frequency dependent differential capacitance is intrinsic to the formation of a layer having an amorphous or strongly disordered nature requiring the use of interpretative models accounting for such a specific feature. According to this it appears preferable to afford such a common aspect in the study of passive film/electrolyte interface by using a more general theoretical approach based on the theory of amorphous semiconductor Schottky barrier which is able to provide a better physical description also for the behavior of crystalline semiconductor junction containing a distribution of donor (acceptor) level in the forbidden energy gap of the SC. 
On the other hand semiconducting passive films in a large range of thickness $(2 \sim 200 \mathrm{~nm})$ can be grown on different valve metals $(\mathrm{Ti}, \mathrm{Ta}, \mathrm{Nb}, \mathrm{W})$ with donor density values of $10^{17} \sim 10^{20}$ $\mathrm{cm}^{-3}$, measured according to M-S equation. For these systems a thickness dependent, as $\left(d_{o x}\right)^{-2}$, donor concentration changing with the growth conditions, initial surface treatment, post-anodizing annealing and nature of investigated metal has been reported by different groups. ${ }^{28,74-75}$ Also for these systems a systematic dependence of the measured capacitance values from the frequency of ac signal has been observed. Usually at constant frequency a transition from semiconductor-like to insulating behavior was reported with increasing film thickness at not too low estimated donor concentration $\left(N_{D}>10^{18} \mathrm{~cm}^{-3}\right),{ }^{74}$ whilst at large film thickness the same transition may occur with increasing ac signal frequency. ${ }^{75-78}$

In this frame the theory of amorphous semiconductor Schottky barrier provides a unified approach to the theory of electrical admittance of electrode/electrolyte junction including as limiting case the ideal SC/electrolyte junction on which the traditional M-S approach is based. The theory and the results of such studies will be presented and discussed in the next Section.

\section{Differential Admittance in Semiconductor/Electrolyte Junction}

In order to understand the main differences in the behavior of a-SC Schottky barrier with respect to the case of crystalline semiconductor it may be helpful to compare preliminarily the density of states distribution in both materials. We stress that usually amorphous materials maintain the same short-range order than their crystalline counterparts and that the main differences come out from the absence of the long-range order, typical of crystalline phases. ${ }^{78-84}$ It is now generally accepted that the band structure model retains its validity also in absence of the long-range lattice periodicity. This means that the long-range disorder perturbs but does not annihilate the band structure: its main effect is the presence of a finite DOS within the so-called mobility gap, $E_{C}-E_{V}$, of the amorphous semiconductor (a-SC) or insulator.

In Fig. 3 we report the model of DOS distribution vs. energy for generic crystalline and amorphous material. Although the gen- 

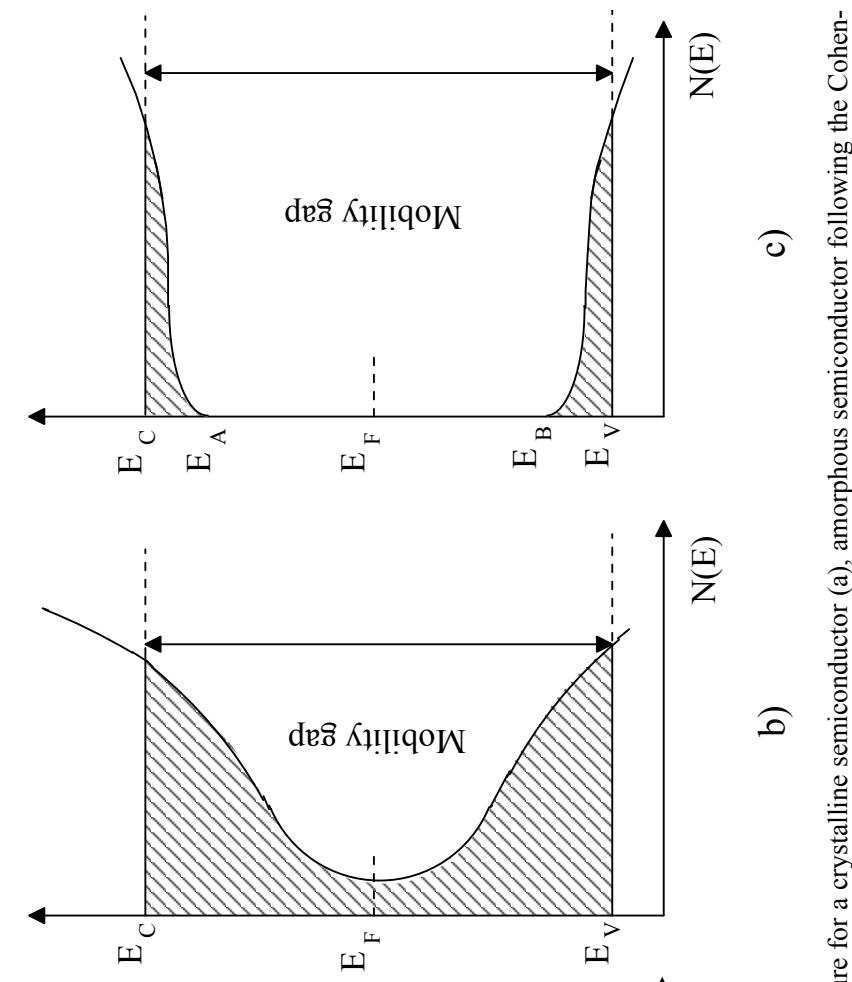

艺

है

ब류

อ

劳

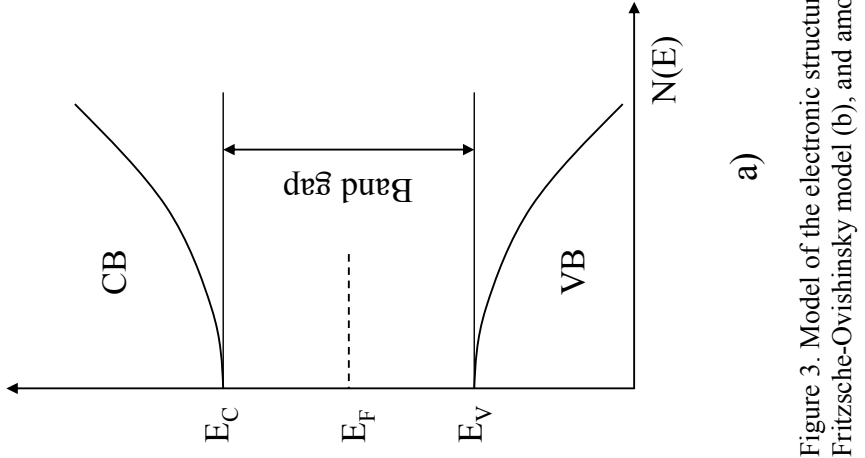


eral features of DOS distribution are preserved, especially if we compare Fig. 3a with Fig. 3c, some differences are evident and they strongly affect the response of a-SC/El junction under ac electrical as well under light stimulus (see below). The DOS distribution of Fig. 3b, initially proposed by Cohen-Fritzsche-Ovishinsky (CFO model) ${ }^{79}$ takes into account the possible existence of lattice defects inside the semiconductor which originate a continuous distribution of electronic states within the mobility gap (like in a$\mathrm{Si}: \mathrm{H})$. On the other hand the DOS distribution of Fig. 3c, due to Mott and Davis, ${ }^{81}$ has been proposed for an ideal amorphous material in which only the long-range lattice disorder is taken into account. Other models have been suggested for explaining the behavior of different classes of amorphous materials, but they involve only minor modifications to those of Fig. 3, when the existence of specific defects in the investigated material is considered.

As evidenced in the Fig. 3, the general features of the DOS of crystalline materials are preserved also for disordered phases, but some differences are now evident with respect to the crystalline $\mathrm{SC}$ and these can be summarized as follows:

(a) Existence of a finite DOS within the mobility gap, defined by two mobility edges, $E_{C}$ and $E_{V}$, in the conduction band (CB) and in the valence band (VB), respectively.

(b) For energy levels below $E_{C}$ or above $E_{V}$, the free electronlike DOS, $N(E) \propto E^{1 / 2}$ is no more generally valid. In these energy regions the presence of a tail of states, exponentially $^{82}$ or linearly ${ }^{81}$ decreasing, has been suggested by different authors for explaining the optical properties of different amorphous materials.

(c) Different mechanisms of charge carriers transport are invoked in extended (below $E_{C}$ or above $E_{V}$ ) or localized (within the mobility gap) electronic states. A free carrierlike mechanism of transport is involved in the first case, whilst a transport by hopping (thermally activated) is assumed in localized states.

These differences between the distributions of electronic states in crystalline and disordered materials have noticeable influence on both the impedance and the photoelectrochemical behavior of the a-SC/El junction. In order to highlight such differences in the impedance behavior of the a-SC/El junction, we will derive some 
of the most important results of the theory of Schottky barriers which are relevant for a better understanding of the difference in admittance behavior of c-SC/electrolyte and passive film/ electrolyte junctions. $^{76,78,85-90}$

It was shown by different authors that the existence of deep electronic states in the mobility gap of the material influences both the shape of the space-charge region and the frequency response of the barrier to the modulating ac signal. ${ }^{85-90}$ As for the first aspect, the potential distribution inside the $\mathrm{SC}$ can be obtained by solving the one dimensional Poisson equation with the suitable boundary conditions:

$$
\frac{\partial^{2} \psi(x)}{\partial x^{2}}=\frac{\rho(x)}{\varepsilon_{o x} \varepsilon_{0}}
$$

where $\psi(x)$ represents the band bending at a point $x$ within the barrier (the galvani potential has opposite sign), and $\rho(x)$ the volumetric density of charge at $x . \rho(x)$ is strictly related to the local potential so that Eq. (14) can be integrated by changing the variable $x$ with $\psi$ :

$$
d\left(\frac{\partial \psi}{\partial x}\right)^{2}=\frac{2}{\varepsilon_{o x} \varepsilon_{0}} \rho(\psi) d \psi
$$

If the space charge region is very small with respect to the total thickness of the semiconductor, the usual boundary conditions for infinite length semiconductor holds:

$$
\begin{array}{ll}
\psi=0 \text { and } \mathrm{d} \psi / \mathrm{dx}=0 & \text { for } x \rightarrow \infty \\
\psi=\psi_{S C} & \text { for } x=0 .
\end{array}
$$

The first integral of Poisson Eq. (15) gives:

$$
\left.\frac{\partial \psi}{\partial x}\right|_{\psi}= \pm\left(\int_{0}^{\psi} \frac{2}{\varepsilon_{o x} \varepsilon_{0}} \rho d \psi^{\prime}\right)^{\frac{1}{2}}= \pm \sqrt{H(\psi)}
$$


where + is used if $\psi$ is negative and - if $\psi$ is positive. By further integration we can find the value of $x$ for different values of band bending in the semiconductor $\psi_{0}$ :

$$
x\left(\psi_{S C}, \psi_{0}\right)=\mp \int_{\psi_{0}}^{\psi_{S C}} \frac{d \psi}{\left(\int_{0}^{\psi} \frac{2}{\varepsilon_{o x} \varepsilon_{0}} \rho d \psi^{\prime}\right)^{\frac{1}{2}}}
$$

The integration of Eq. (17) in the hypothesis of a constant DOS distribution, $N$, has been carried out in reference ${ }^{85}$ and it provides the dependence of band bending on the distance from the semiconductor/electrolyte interface as:

$$
\psi(x)=\psi_{S C} e^{-x / x_{0}}
$$

where $x_{0}=\left(\varepsilon_{\mathrm{ox}} \varepsilon_{0} / e^{2} N\right)$ and $N$ is the DOS in $\mathrm{cm}^{-3} \mathrm{eV}^{-1}$.

The charge per unit area stored in the semiconductor, $Q_{S C}$, is equal to:

$$
Q_{S C}=\int_{0}^{\infty} \rho d x=\varepsilon_{o x} \varepsilon_{0} \int_{0}^{\infty} d \frac{\partial \psi}{\partial x}=-\left.\varepsilon_{o x} \varepsilon_{0} \frac{\partial \psi}{\partial x}\right|_{0}
$$

The general expression of the steady state space charge capacitance of the junction is given by:

$$
C_{S C}\left(\psi_{S C}, 0\right)=-\varepsilon_{o x} \varepsilon_{0} \frac{d}{d \psi_{S}}\left(\left.\frac{\partial \psi}{\partial x}\right|_{0}\right)=\mp\left(\frac{\varepsilon_{O x} \varepsilon_{0}}{2}\right)^{\frac{1}{2}} \frac{\rho\left(\psi_{S}\right)}{\left(\int_{0}^{\psi_{S}} \rho\left(\psi^{\prime}\right) d \psi^{\prime}\right)^{\frac{1}{2}}}
$$


As expected, the response of the semiconductor is dependent on $\rho$ which is a function of the band bending and charge distribution into localized states within the forbidden gap (mobility gap) of the crystalline (amorphous) semiconductor.

The dependence of the density of charge on the band bending for a n-type c-SC with deep traps, behaving as donor-like levels, can be written as:

$$
\begin{array}{r}
\rho(\psi)=|e| \sum_{i=1}^{m_{T}} N_{T, i}\left[\frac{\exp \left(\frac{E_{T, i}+|e| \psi-E_{F}}{k_{B} T}\right)}{1+\exp \left(\frac{E_{T, i}+|e| \psi-E_{F}}{k_{B} T}\right)}\right. \\
\left.-\frac{\exp \left(\frac{E_{T, i}-|e| \psi-E_{F}}{k_{B} T}\right)}{1+\exp \left(\frac{E_{T, i}-E_{F}}{k_{B} T}\right)}\right]
\end{array}
$$

where $E_{T, i}$ is the energy level of the i-th deep trap and $N_{T, i}$ the concentration of deep traps at $E_{T, i}$ in $\mathrm{cm}^{-3}$. It can be easily shown that the previous equations give, as particular case, the M-S equation once the expression of the volumetric density of charge for crystalline SC as a function of the band bending is substituted in Eq. (20). In fact for a single donor-like level completely ionized $\left(E_{T, i}-E_{F}\right.$ $>$ 0) Eq. (21) reduces to the Mott-Schottky case:

$$
\rho(\psi)=|e| N_{D}\left[1-\exp \left(-\frac{|e| \psi}{k_{B} T}\right)\right]
$$

By using Eq. (22) in Eq. (20), after simple algebraic manipulation, the M-S equation, previously reported (Eq. 8), is obtained. In this last case the frequency dependence in Eq. (20) is absent owing to the fact that there is no delay in following the $a c$ signal by the electrons staying in extended states of the conduction (or valence band), whichever is the chosen frequency of the modulating $a c$ signal. 
On the other hand by using Eq. (21) together with Eq. (20) the static space charge capacitance for an n-type semiconductor with multiple deep donor levels is obtained as reported in a previous work. ${ }^{91}$ Such an expression does not take into account the finite time of answer of electrons lying in deep trap ${ }^{71,72}$ so that a new model which accounts for the frequency dependence of the a-SC Schottky barrier containing deep donor levels and/or a distributed density of states within the mobility gap of a-SC was successively put forward by the same authors in agreement with the theory of aSC Schottky barrier. ${ }^{92}$

\section{Static Differential Capacitance in a-Semiconductor with Constant DOS}

In Fig. 4 the energetics of n-type a-SC/El interface is reported, under the simplifying hypothesis of a spatially homogeneous a-SC having a constant DOS distribution in energy into the mobility gap. Both the assumptions (homogeneity and constancy in energy) are necessary to get an analytical solution for the general expression of $C_{S C}$ in a-SC Schottky barrier ${ }^{85-90}$ Both these assumptions will be relaxed in discussing the admittance behavior of passive film/electrolyte junction.

Owing to a large density of localized states within the mobility gap we can assume as negligible the contribution of the free electrons in the conduction band $\left(E_{C}-E_{F}>3 k T\right)$ to the density of charge $\rho(\psi)$. Moreover an abrupt change in the electron occupancy of the localized states at the Fermi level is assumed. ${ }^{86}$ The electric charge density for a-SC can be written, within the given assumption, as:

$$
\rho(\psi)=|e| \int_{E_{F}-|e| \psi}^{E_{F}} N(E) d E=|e| N \psi
$$

The contribution of minority carriers to the density of charge is assumed negligible, whilst the electron quasi Fermi level is assumed flat throughout the semiconductor as long as the band bend- 


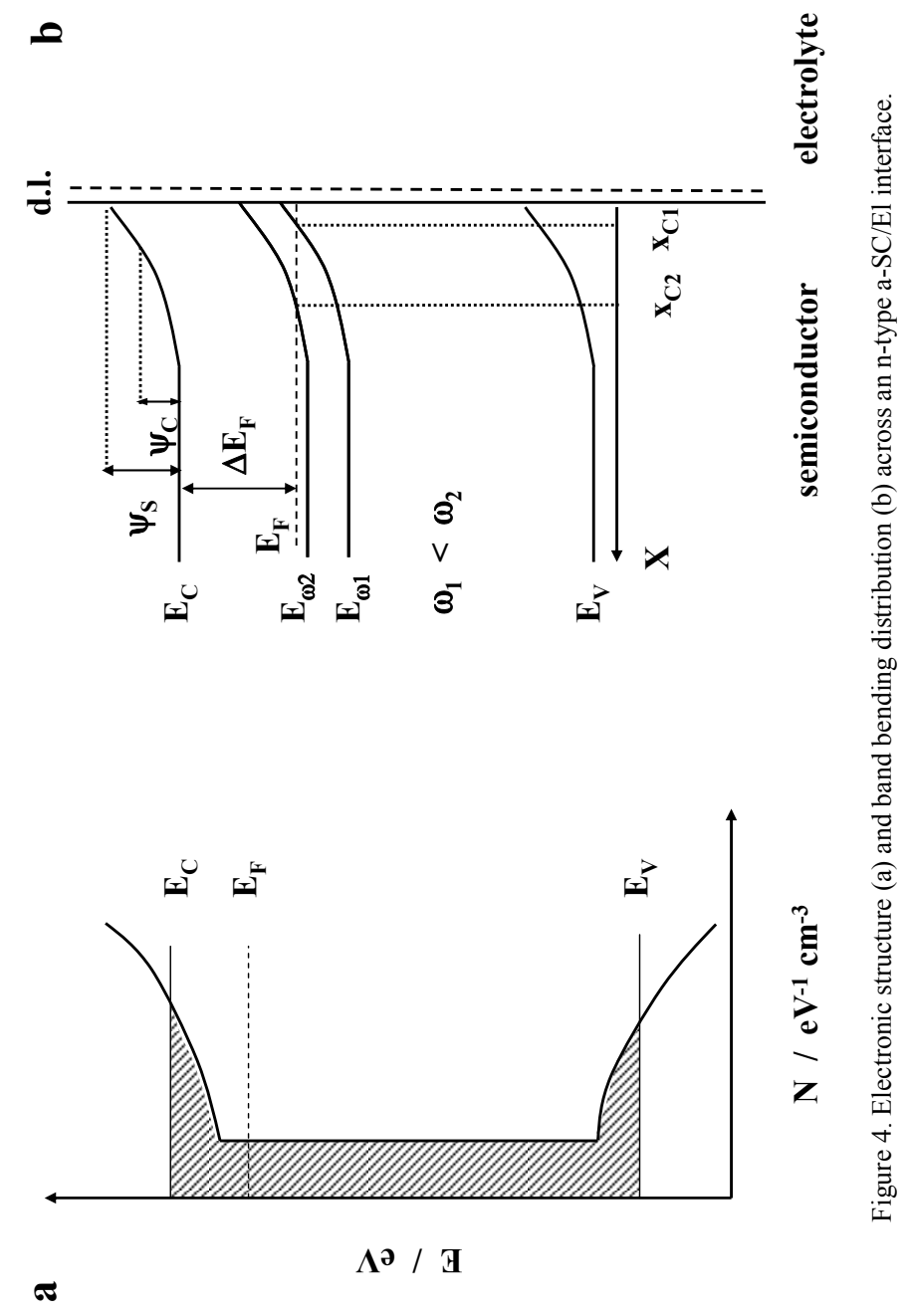


ing is not too high (see below). Under these conditions the static capacitance (see Eq. 20) can be calculated as:

$$
C_{S C}\left(\psi_{S C}, 0\right)=\left(\varepsilon_{o x} \varepsilon_{0}|e|^{2} N\right)^{1 / 2}
$$

It may be useful to compare the results for $C_{S C}$ obtained for aSC with those reported by Gerischer ${ }^{69}$ for graphite as well as the extension of space charge layer in amorphous semiconductor with constant DOS: $N(E)=N$. By assuming the space charge region as the distance inside the a-SC where the band bending is in the order of $k T / e$ we can derive from Eq. (17) the extension of $X_{S C}$ as:

$$
X_{S C}=X_{0} \ln \left(\frac{|e| \psi_{S C}}{k T}\right) \quad \text { for }|e| \psi_{\mathrm{SC}}>3 k T
$$

By assuming a DOS at $\mathrm{E}_{\mathrm{F}}$ around $10^{20} \mathrm{eV}^{-1} \mathrm{~cm}^{-3}$ we get for $C_{S C}$ a constant value of $3.8 \mu \mathrm{F} \mathrm{cm}^{-2}$ and a space charge length $X_{S C}$ $=5.4 \mathrm{~nm}$ for a band bending around $0.25 \mathrm{~V}$ with $\varepsilon_{\mathrm{ox}}=10$. We like to stress that in the case of base metals like: $\mathrm{Ni}, \mathrm{Fe}$, and $\mathrm{Cr}$ the value of $X_{S C}$ at $0.25 \mathrm{~V}$ of band bending results higher than the thickness of passive film reported in literature for such metals. If we take into account that the value of $C_{S C}$ is of the same order of the Helmholtz double layer and that small changes in $C_{H}$ as well as in the value of dielectric constant of the passive film can occur with changing potential during the capacitance measurements it is, once more, necessary to be aware that the rather unusual donor (acceptor) density measured on these systems could be affected by different pitfalls in the assumed physical model.

In order to evidence the main differences in the admittance behavior of a-SC with respect the crystalline counterpart it is necessary to derive, however, the expression of differential admittance in a-SC-Schottky barrier as a function of the frequency of $a c$ signal. 


\section{AC Response of a-Semiconductor Schottky Barrier}

According to the schematic DOS distribution reported in Fig. 4 the most part of electronic charge is now located below the Fermi level in localized states quite distant in energy from the conduction band mobility edge separating the extended states region from localized ones. According to the theory, ${ }^{85-90}$ at variance with the case of crystalline SC, the filled electronic states into the gap (see Fig. 4b) do not follow instantaneously the imposed $a c$ signal, but they need a finite response time. This response time depends on their energy position with respect to the Fermi level and it can be much longer than the period of the $a c$ signal having angular frequency $\omega$. In fact the relaxation time, $\tau$, for the capture/emission of electrons from electronic states $E$ below $E_{F}$ is assumed to follow the relationship:

$$
\tau=\tau_{0} \exp \left(\frac{E_{C}-E}{k T}\right)
$$

where, at constant temperature, $\tau_{0}$ is a constant characteristic of each material usually ranging between $10^{-14} \sim 10^{-10} \mathrm{~s}$. According to Eq. (26), by decreasing the energy of the localized state in the gap, $\tau$ increases sharply so that deep states (for which $\omega \tau>>1$ ) do not respond to the ac signal.

By assuming a full response for states satisfying the condition $\omega \tau<<1$ and a null response for states having $\omega \tau>>1$, a sharp cutoff energy level, $E_{\omega}$, separating states responding from those not responding to the signal, can be defined from the condition: $\omega \tau=1$.

The location of the cut-off level $E_{\omega}$ is found by imposing in Eq. (26) $\omega \tau=1$ for $E=E_{\omega}$, which gives:

$$
E_{C}-E_{\omega}=-k T \ln \left(\omega \tau_{0}\right)
$$

This condition occurs at some position within the barrier $(X=$ $X_{C}$ ) which is a function of band bending and $a c$ frequency (see Fig. $4 b)$. The intersection of the cut-off level, $E_{\omega}$, with the Fermi level allows to locate the point within the barrier, $\mathrm{X}_{\mathrm{C}}$, which separates 
two regions of the a-SC (see Fig. 4b). The first region (for $X>X_{C}$ ) represents the region where all electronic states fully respond to the $a c$ signal $(\omega \tau<<1)$, the second one (for $\left.X<X_{C}\right)$ where they do not respond at all $(\omega \tau>>1)$. The corresponding band bending at $X_{C}$ is given according to Fig. $4 \mathrm{~b}$ by:

$$
|e| \psi_{C}=-k T \ln \left(\omega \tau_{0}\right)-\Delta E_{F}
$$

with $\Delta E_{F}=\left(E_{C}-E_{F}\right)_{\text {bulk. }}$. We like to stress that $X_{C}$ is now a distance in the barrier which changes with changing frequency, $\omega$, and band bending $\psi_{\mathrm{s}}$. In particular $X_{C}$ increases with increasing frequency, at constant polarization, or with increasing polarization at constant frequency. From the theory it comes out that the total capacitance is sum of two series contribution coming from the $X<X_{C}$ and $X>$ $X_{C}$ regions of the a-SC. The contribution to the conductance comes mainly from the region around $X=X_{C}$ dividing the total response from null response regions. In the hypothesis of a constant DOS the total capacitance is given by the sum of the two contributions $^{77,78,93,94}$ (see Fig. 5):

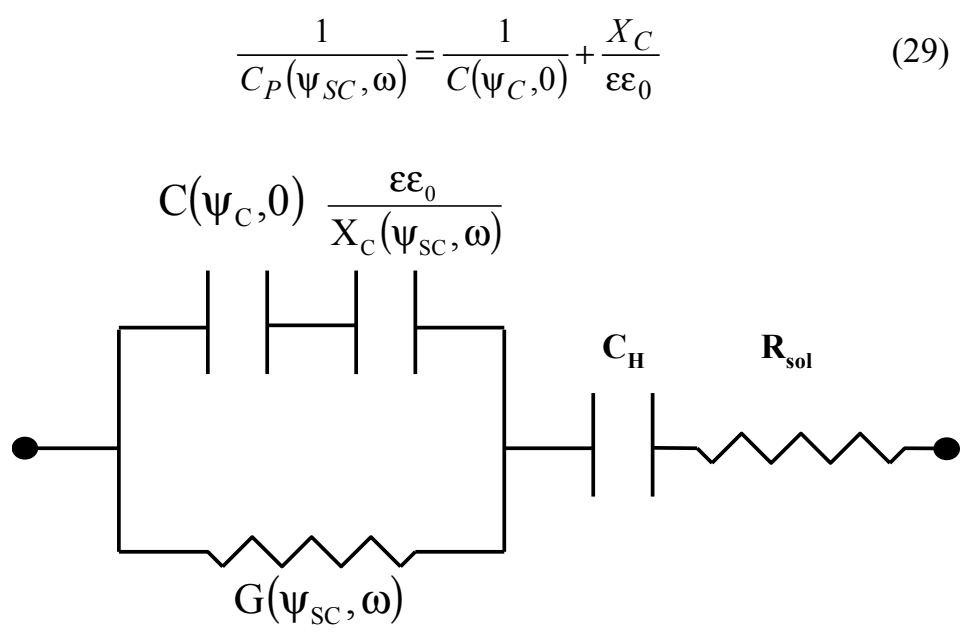

Figure 5. Equivalent electrical circuit of an ideally polarizable a-SC/electrolyte interface in absence of surface state contribution. 
where $C\left(\psi_{C}, 0\right)=\sqrt{\varepsilon \varepsilon_{0} e^{2} N}$ coincides with the static space charge capacitance of a-SC previously derived and it is frequency independent whilst $X_{C}=\sqrt{\frac{\varepsilon \varepsilon_{0}}{e^{2} N}} \ln \frac{\psi_{S C}}{\psi_{C}}$ is the distance from the surface of the barrier at which the Fermi level crosses the cut-off energy level $\mathrm{E}_{\omega}$ inside the space charge region. After substitution the following relationship is obtained for the total capacitance:

$$
C_{L B B}\left(\psi_{S C}, \omega\right)=\sqrt{\varepsilon \varepsilon_{0} e^{2} N}\left(1+\ln \frac{\psi_{S C}}{\psi_{C}}\right)^{-1}
$$

while the parallel conductance of the junction is given by:

$$
G_{L B B}\left(\psi_{S C}, \omega\right)=\left(\frac{\omega \pi k T}{2}|e| \psi_{C}\right) \sqrt{\varepsilon \varepsilon_{0} e^{2} N}\left(1+\ln \frac{\psi_{S C}}{\psi_{C}}\right)^{-2}
$$

The analytical solutions for the admittance components of the junction have been derived under conditions that $\psi_{\mathrm{SC}}>\psi_{\mathrm{C}}>$ $3 k T /|e|$ and at not too high band bending (Low Band Bending regime). ${ }^{76-78}$

It has been shown that $G\left(\psi_{S C}, \omega\right)$ has a spectroscopic character with respect to the distribution of electronic states within the gap, whilst variations in DOS cause only minor changes in the $C\left(\psi_{S C}, \omega\right)$ vs. potential plots provided that the DOS varies little over an energy range of $k T$. At variance with the M-S analysis in the case of a-SC a fitting procedure is required for getting flat band potential and DOS distribution around the Fermi level. As reported in previous works ${ }^{77-78}$ the fitting must be carried out on both components of the admittance of the junction. For an ideally polarizable a-SC/electrolyte interface and in absence of surface state contribution to the measured admittance the electrical equivalent circuit of such an interface is shown in Fig. 5. Such an equivalent circuit has been used by the authors for fitting both the components of space charge admittance, $Y_{S C}$, at high frequency when the contribution of surface state admittance, $Y_{S S}$, is negligible with respect to $Y_{S C}$. The fitting procedure must be performed under 
condition that at any employed $a c$ frequency both the $1 / C_{S C}$ vs. $\psi_{S C}$ and the $G_{S C}$ vs. $\psi_{S C}$ plots give the same $U_{f b}$ value, within an assigned uncertainty (in our case, $0.025 \mathrm{~V}$ ). Moreover an additional constraint arises from Eq. (28) requiring for $\psi_{C}$ a variation of 59 $\mathrm{mV}$ for decade of frequency at room temperature. ${ }^{76-78,93-98}$

As reported previously ${ }^{76-78}$ a very meaningful difference with respect to the use of M-S analysis is the procedure for locating the mobility edge, $E_{C}$ (n-type) or $E_{V}$ (p-type), by considering that now the most part of electronic charge is located into the localized states below the Fermi level. At this aim instead of Eq. (11) valid for c-SC we can make use of Eq. (28) once the $\psi_{C}$ parameter and flat band potential $U_{f b}$ have been obtained. In order to perform such a task we need to know for each material the constant $\tau_{0}$. For our purposes an average $\tau_{0}$ value of $10^{-12} \mathrm{~s}$ can be assumed in absence of further information. This choice could affect the location of the mobility edge $\left(E_{C}\right.$ or $\left.E_{V}\right)$ by a quantity equal to $0.12 \mathrm{eV}$ which is not too bad if we take into account the absence of a sharp boundary between localized and extended states region.

The ability of previous admittance equations, in low band banding approximation, to fit the experimental curves of different amorphous passive film SC/electrolyte junctions has been tested in previous works. ${ }^{76-78}$ It is in our opinion that such an approximation could be generally employed for analysing the admittance behavior of thin passive films on base metal $(\mathrm{Fe}, \mathrm{Ni}, \mathrm{Cr})$ where it is expected that the space charge region reaches the metal interface at rather low values of band bending (see above for estimates of $X_{S C}$ ) for reasonable values of DOS $\left(10^{20} \mathrm{~cm}^{-3} \mathrm{eV}^{-1}\right)$ near the Fermi level.

In the case of thicker films like those grown on valve metals ( Ti, Nb, W, Ta, etc.) may be of interest for a better understanding of the mechanism of growth of the film and for deriving information on the DOS distribution inside the a-SC to investigate the admittance behavior of the passive film/electrolyte junction in a larger range of band bending values. At high band bending a parabolic potential distribution will appear in the deep depletion region at the surface of a-SC/El junctions where the quasi-Fermi level of the a-SC is now pinned, due to the equilibrium of the emission rate for electrons and holes from localized states to the respective band 


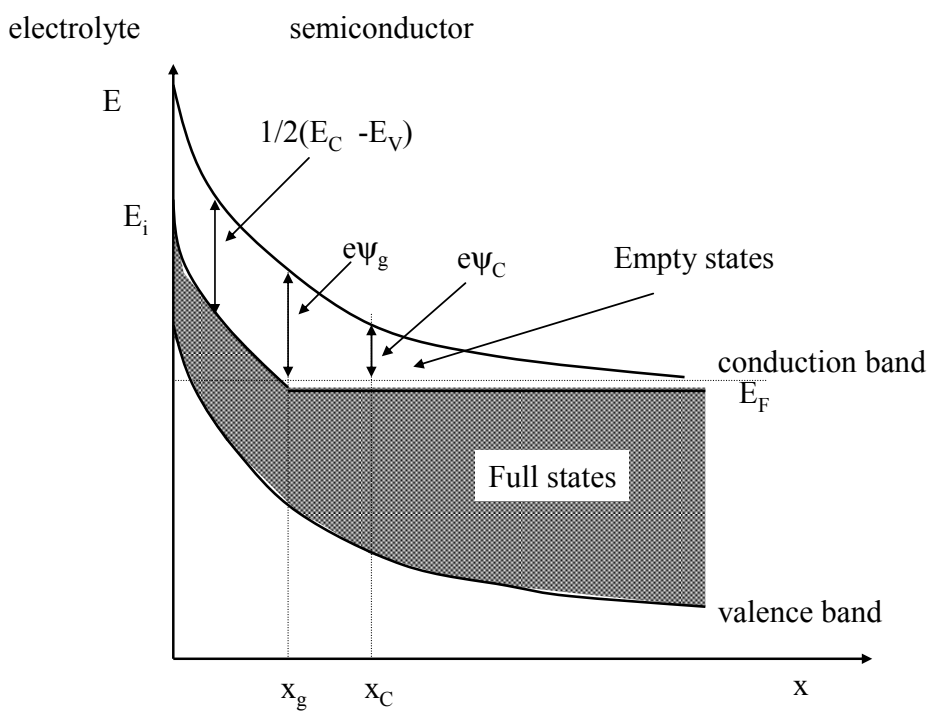

Figure 6. Band bending distribution across an n-type a-SC/E1 interface under the conditions of high band bending (HBB).

edge. It is possible to define a band banding $\psi_{g}$ (see Fig. 6) corresponding to the equilibrium of emission/capture process:

$$
|e| \psi_{g}=\frac{E_{g}}{2}+\frac{k_{B} T}{2} \ln \left(\frac{v_{n}}{v_{p}}\right)-\Delta E_{F}
$$

The point at which the band bending of the semiconductor is equal to $\psi_{g}$ is $x_{g}$ and it can be obtained from Eq. (17) by imposing $\psi_{0}=\psi_{g}$. In order to include the deep depletion region the previous equations have been modified ${ }^{95-98}$ as follows:

$$
\frac{1}{C_{H B B}\left(\psi_{S C}, \omega\right)}=\frac{1}{\sqrt{\varepsilon \varepsilon_{0} e^{2} N}}\left(\ln \frac{\psi_{g}}{\psi_{C}}+\sqrt{1+\frac{2}{\psi_{g}}\left(\psi_{S C}-\psi_{g}\right)}\right)
$$




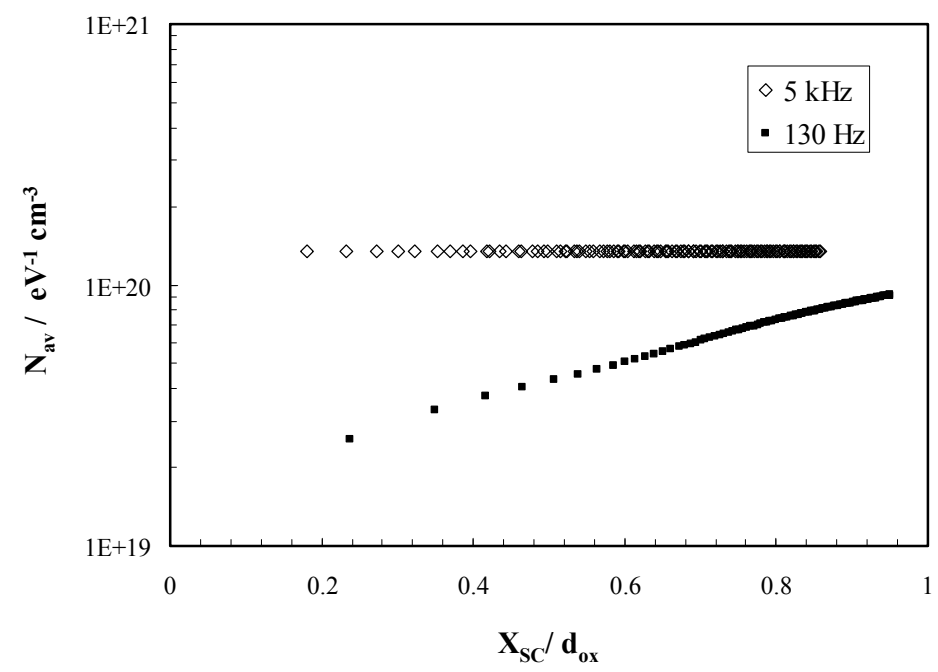

Figure 7. DOS distribution for a- $\mathrm{WO}_{3}$ obtained from Eq. (33) as a function of the position inside the passive film at different frequencies.

$$
\begin{aligned}
G_{H B B}\left(\psi_{S C}, \omega\right) & =\pi^{2} f \frac{k T}{|e| \psi_{C}} \sqrt{\varepsilon \varepsilon_{0} e^{2} N} \\
& \times\left(\ln \frac{\psi_{g}}{\psi_{C}}+\sqrt{1+\frac{2}{\psi_{g}}\left(\psi_{S C}-\psi_{g}\right)}\right)^{-2}
\end{aligned}
$$

Equations (33) and (34) have been derived under the same conditions valid for the low band bending (LBB) expression and they coincide with the previous ones for $\psi_{S C}=\psi_{g}$. The first test on the ability of Eqs. (33) and (34) to fit the two components of the differential admittance in a relatively large range of band bending values was carried out for passive film on $\mathrm{WO}_{3}$ and $\mathrm{Nb}_{2} \mathrm{O}_{5}$ about $25 \mathrm{~nm}$ thick. ${ }^{94}$ It was noticeable, in the case of $\mathrm{WO}_{3}$, that from Eq. (33) an almost constant average DOS equal to about $10^{20} \mathrm{eV}^{-1} \mathrm{~cm}^{-3}$ was derived for $\mathrm{a}-\mathrm{WO}_{3}$ in agreement with previous results, obtained in the low band bending approximation. ${ }^{78}$ However a more close inspection of the admittance behavior at different scan rates 
and $a c$ frequencies suggested the possible presence of a more complex dependence of localized DOS both from energy as well as from the spatial coordinate (metal/oxide interface distance) (see Fig. 7).

In more recent papers ${ }^{95-97}$ a study aimed to get information on the possible spatial and energy dependence of DOS near the Fermi level of a-SC has been carried out trough a detailed investigation of a- $\mathrm{Nb}_{2} \mathrm{O}_{5}$ passive films anodically grown. The choice of a- $\mathrm{Nb}_{2} \mathrm{O}_{5}$ was dictated by its long term stability in aqueous solution of variable $\mathrm{pH}$ and by the fact that anodic film of $\mathrm{Nb}_{2} \mathrm{O}_{5}$ are amorphous and easily prepared in a wide range of film thickness, from few to hundredths of nanometers. These aspects make $\mathrm{a}-\mathrm{Nb}_{2} \mathrm{O}_{5}$ an ideal candidate for testing any proposed model of a-SC/electrolyte junction. From such studies it comes out the importance of the choice of the equivalent circuit to be used to subtract from the measured admittance the components of the admittance depending on the electrochemical reactions at the a-SC/electrolyte interface by including also the presence of intrinsic surface states which could result from the amorphous nature of the material (see Fig. 8).

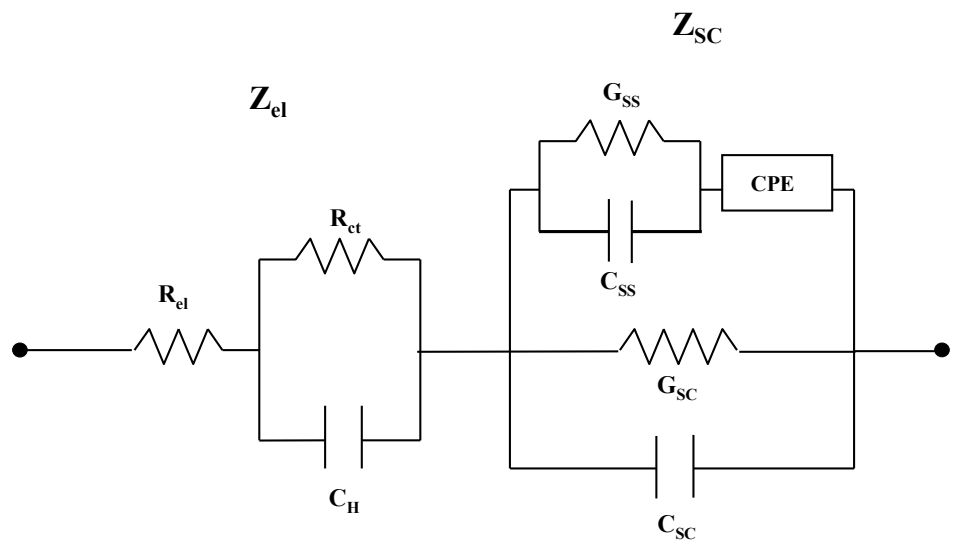

Figure 8. Equivalent electrical circuit of an a-SC/electrolyte interface, considering the contribution of an electrochemical reaction occurring through electrons/holes exchange with the surface states. 


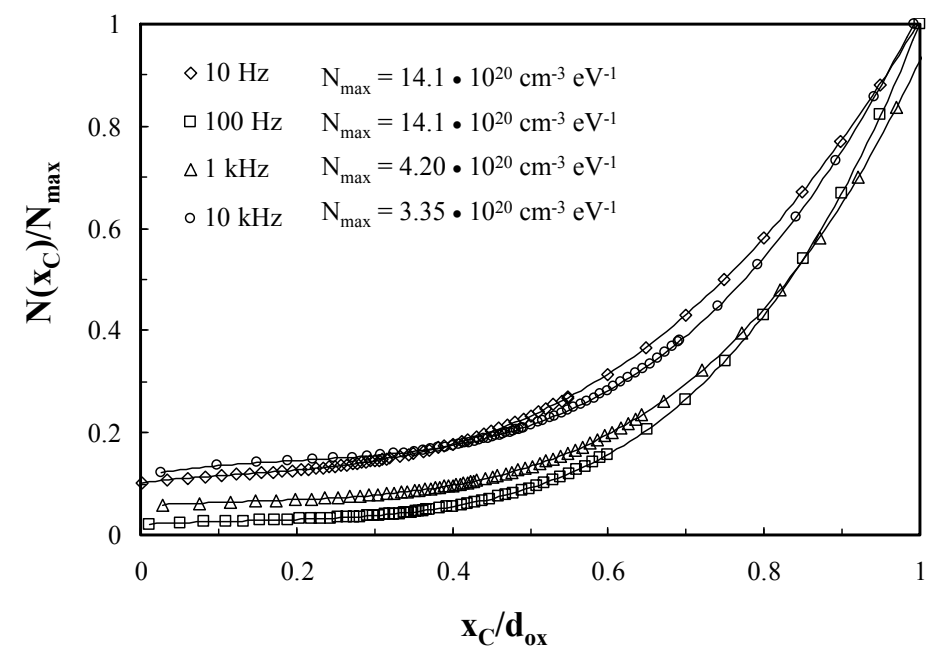

Figure 9. DOS distribution for a- $\mathrm{Nb}_{2} \mathrm{O}_{5}$ obtained from Eq. (36) as function of the position inside the passive film at different frequencies. Reprinted from F. Di Quarto, F. La Mantia, and M. Santamaria, "Physicochemical characterization of passive films on niobium by admittance and electrochemical impedance spectroscopy studies." Electrochim. Acta 50 (2005) 5090, Copyright (2005) with permission from Elsevier.

Moreover it came out that in order to fit the EIS spectra of the junction in the overall investigated range of frequency $(0.1 \mathrm{~Hz}-$ $100 \mathrm{kHz})$ as well the two components- $C_{p}(\omega, \psi), G_{p}(\omega, \psi)-$ of the admittance curves in a wide range of frequencies $(10 \mathrm{~Hz}-10 \mathrm{kHz})$ and electrode potentials $(\sim 5 \mathrm{~V})$ a DOS distribution slightly changing with energy and along the spatial coordinate was necessary (see Fig. 9 and 10).

As for the spatial dependence of DOS a fitting of the admittance curves, $C_{S C}\left(\omega, \psi_{\mathrm{S}}\right)$ and $G_{S C}\left(\omega, \psi_{\mathrm{S}}\right)$, at different frequencies $(10 \mathrm{~Hz} \leq f \leq 10 \mathrm{kHz})$, was carried out ${ }^{95-97}$ by using Eqs. (33) and (34) modified as:

$$
\begin{aligned}
& \frac{1}{C_{S C}\left(x, \psi_{S C}, \omega\right)}=\frac{1}{C\left(\psi_{S C}, \omega\right)} \mathrm{f}_{\omega}\left(x\left(\psi_{S C}\right)\right) \\
& G_{S C}\left(x, \psi_{S C}, \omega\right)=G\left(\psi_{S C}, \omega\right) g_{\omega}\left(x\left(\psi_{S C}\right)\right)
\end{aligned}
$$




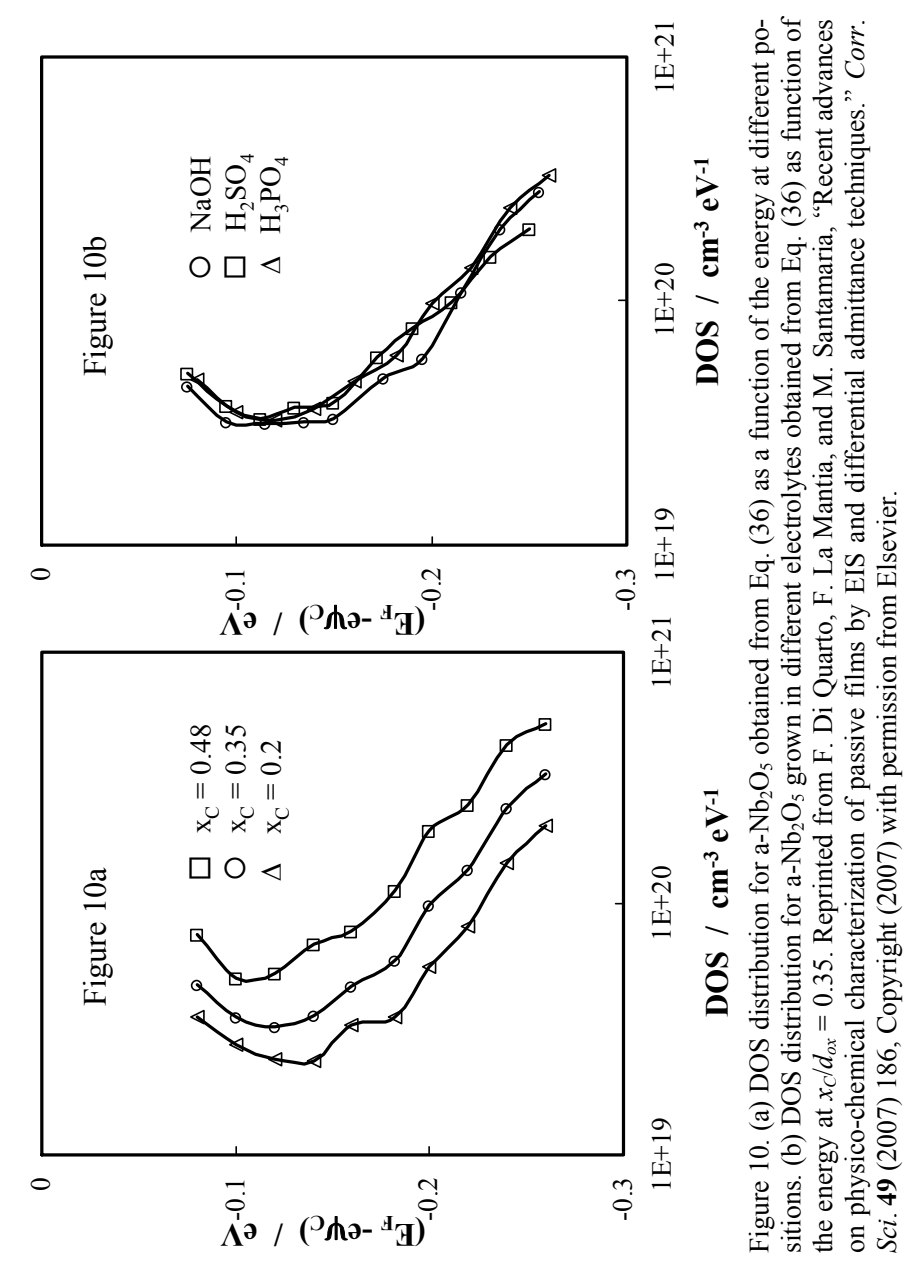


where $f_{\omega}\left(x\left(\psi_{\mathrm{s}}\right)\right)$ and $g_{\omega}\left(x\left(\psi_{\mathrm{s}}\right)\right)$ are respectively two different trial functions depending only on the electrode potential but changing with employed frequency. The term multiplying the two trial functions can be considered as coincident with the expression of $\left(C_{S C}\right)^{-}$ ${ }^{1}$ and $\mathrm{G}_{\mathrm{SC}}$ in absence of spatial variation in DOS (homogeneous film) but averaged in energy $\left(N(E)=N_{a v}\right)$.

By using the spectroscopic character of the conductance in aSC Schottky barrier, ${ }^{87,88}$ the DOS at $x_{\mathrm{C}}, N\left(E_{F}-\mathrm{e} \psi_{C}\right)$, was obtained by using the following equation: ${ }^{95}$

$$
\begin{gathered}
N\left(E_{F}-|e| \psi_{C}\right)=\frac{2}{\pi k T} \frac{G_{S C}\left(\psi_{S C}, \omega\right) / \omega}{C_{S C}^{2}\left(\psi_{S C}, \omega\right)} \mathrm{f}_{\omega}\left(x\left(\psi_{S C}\right)\right) C\left(\psi_{C}, 0\right) \\
\times \int_{E_{F}-|e| \psi_{C}}^{E_{F}} N(E) d E
\end{gathered}
$$

and by substituting to the integral the term $e \psi_{c} N_{a v}$. As first approximation for $N_{a v}$ that one derived from the fitting of $C_{S C}$ at the same $\omega$ and $x_{\mathrm{C}}$ have been chosen. As mentioned in previous work, ${ }^{94,97}$ the choice of the other fitting parameters $\left(U_{f b}, \psi_{g}, \psi_{C}\right)$ could be carried out through an educated guess procedure by taking into account that:

- as for the $\psi_{C}$ parameter the constraint of a decrease of 0.06 $\mathrm{eV}$ for decade of frequency has to be introduced in the fitting procedure. A check of the acceptability of the $\psi_{\mathrm{C}}$ choice is the prediction of flattening of capacitance curves at a frequency corresponding to $\psi_{C}=0$;

- in absence of further information the $\psi_{g}$ parameter can be calculated by assuming an equal emission-capture time constant for electrons and hole in the conduction and in valence band (Eq. 32) and by using the measured mobility gap for passive film (a mobility gap equal to $3.40 \mathrm{eV}$ was used for $\mathrm{a}-\mathrm{Nb}_{2} \mathrm{O}_{5}$ film);

- the $U_{f b}$ value derived from fitting the $C_{S C}\left(x, \omega, \psi_{S}\right)$ and $G_{S C}\left(x, \omega, \psi_{\mathrm{S}}\right)$ curves should have to differ no more than $k_{B} T / e$ volt. 
The DOS distribution in a- $\mathrm{Nb}_{2} \mathrm{O}_{5}$ grown in different electrolytic solutions as a function of energy and distance from the film/solution interface are reported in Fig. 10a. The results suggest that the DOS in anodic film is changing with distance in energy from the Fermi level but such a distribution of DOS is largely independent from the nature of the electrolytic solution ${ }^{96,97}$ (see Fig. 10b). It was worth to note that almost coincident values of DOS, as a function of the energy distance from the Fermi level, were derived from EIS analysis and DA measurements thus confirming the spectroscopic character of the admittance measurements as a function of the ac signal frequency. We like to stress that, at variance with the simple M-S approach, in the frame of the theory of amorphous Schottky barrier the frequency dependence of the differential admittance curves is explicitly taken into account and the study of such dependence is able to provide further information on the DOS distribution in energy. As for the spatial dependence it seems that a DOS distribution steadily increasing on going from the oxide/electrolyte interface toward the metal/oxide interface is compatible with the experimental results. Such a finding seems quite reasonable if it is assumed that the DOS distribution is related to the anodizing process and in particular to some specific mobile defects becoming frozen when the anodizing process is stopped. An exact quantitative analysis of these aspects needs to solve the problem of taking into account the effects of a finite film thickness on the measured values of admittance particularly in the case of thin films where the space charge region could reach the total film thickness. However if carefully used the DA measurements can provide useful information, also in a quantitative way, on the electronic structure of passive film beside the ordinary estimate of the flat band potential which in dubious cases should be confirmed by other technique like photocurrent spectroscopy (see below). This last technique in presence of insulating passive film is a possible route to the estimation of the flat band potential of the passive film/electrolyte junction and a complementary way to get information on the solid-state properties of passive film as will be discussed in the next Section. 


\section{PHOTOCURRENT SPECTROSCOPY IN PASSIVITY STUDIES}

The first experimental report on the interaction between light and passive films on metal can be traced to the Becquerel's study of photoeffects at metal-electrolyte interface dating back to the first half of $19^{\text {th }}$ century. ${ }^{99}$ Earlier studies on photoelectrochemical behavior of oxidized metals were summarized in a review of the initial $1940 \mathrm{~s}^{100}$ but the beginning of photoelectrochemical science is marked by the work of Brattain and Garret in the mid-1950. ${ }^{15}$ However, the first attempt to use photoelectrochemical technique in passivity studies dates more recently to the end of 1960s when Oshe and Rozenfeld ${ }^{101}$ proposed to characterize the nature of passive films on metals and alloys by using a photopotential method initially proposed by Williams ${ }^{102}$ for bulk semiconductors. Some applications of such a method in passivity studies can be find in Refs. [103, 104] and in references therein. The inadequacy of Oshe and Rozenfeld's method in characterising complex metal/oxide/electrolyte interfaces was initially evidenced by Hackerman et al. ${ }^{105}$ This fact and the onset of more refined theories of photocurrent generation at illuminated semiconductor/electrolyte interface in the mid-1970 ${ }^{106-107}$ made obsolete the Oshe and Rozenfeld's method of characterisation of passive metal/electrolyte interface. In fact, as a result of an intensive research effort on the photoelectrochemical behavior of semiconductor electrodes, aimed to harvest solar energy by photoelectrochemical solar cells, different electrochemical techniques started to be exploited as analytical tools for in situ characterisation of semiconductor/electrolyte (SC/El) interface. ${ }^{108-110}$ In many cases the investigated photoelectrodes were oxides so that it was evident to electrochemists that passive films and corrosion layers having semiconducting or insulating behavior could be scrutinised by using the same techniques used for studying $\mathrm{SC} / \mathrm{El}$ interface. ${ }^{37-40}$

It is worth to note that photoelectrochemical techniques were practically absent in the passivity meeting held in Airlie ${ }^{3}$ but they gained importance since the fifth Symposium on Passivity of Metals and Semiconductors held in $1983 .^{4}$ Since this last symposium PCS technique became a constant presence at passivity meetings and workshops around the world. This is due to the fact that PCS is able to provide information on: 
(1) the energetics at the metal/passive-film/electrolyte interfaces (flat band potential determination, conduction and valence band edges location, internal photo-emission thresholds);

(2) the electronic structure and indirectly (through the optical band gap values) chemical composition of passive films in situ and under controlled potential in long lasting experiments.

With respect to other optical techniques PCS offers the further advantage that the photocurrent response of the passive film is directly related to the amount of absorbed photons. This means that the technique is not demanding in terms of surface finishing so allowing the monitoring of long lasting corrosion processes, where changes of surface reflectivity are expected owing to possible roughening of metal surfaces covered by corrosion products. As for the risk of electrode modifications under illumination, it can be minimised by improving the sensitivity of the signal detection by using a lock-in amplifier, coupled to a mechanical light chopper, which allows scrutinizing very thin films (1-2 nm thick) also under relatively low intensity photon irradiation. More details on the use of Lock-in technique to measure photocurrent signal can be found in Refs. [39, 40] and references therein. PCS presents limitations owing to the following aspects:

(1) the technique is able to scrutinize only photoactive passive films and corrosion layers;

(2) the investigation of surface layers having optical band gap lower than $1.0 \mathrm{eV}$ or larger than $5.5 \mathrm{eV}$ requires special setup or they are experimentally not accessible in aqueous solutions;

(3) structural information and chemical composition of the layers are not accessible directly and complementary investigation based on other techniques can be required.

The first two limitations are rather apparent than real. With the exception of noble metals (Ir, Ru, etc.) which are covered by conducting oxides only at high electrode potentials, most of metals are thermodynamically unstable by immersion in aqueous solution, and they become covered by oxide or hydroxide films having often insulating or semiconducting properties. Moreover, with the ex- 
ception of very few oxides grown on metals of lower electronegativity, the most common base metal oxides have band gap values largely lying within the optical window experimentally accessible by PCS.

The third limitation is the principal one but it has been shown by different authors in the last years that PCS can provide indirectly compositional information once some interpretative model of the photo-electrochemical behavior of passive film/electrolyte interface is introduced which accounts also for the complex electronic structure of amorphous materials. ${ }^{35-39}$ Complementary information accessible by other in situ and ex situ techniques can help this task and may be unavoidable for very complex systems (see below). The aim of this Section is to:

(a) provide a general interpretative framework of the photoelectrochemical behavior of passive metal electrodes by discussing some features which are related to the extreme thinness and/or amorphous nature of the passivating layers;

(b) highlight the more recent quantitative use of PCS for characterising the chemical nature of passive films and corrosion layers.

A very short theoretical background on the photoelectrochemistry of crystalline semiconductors will be provided for readers not acquainted to the subject, in order to evidence the differences between the photoelectrochemical behavior of passive films and bulk crystalline semiconductors. More extensive and detailed introduction to the principles of photoelectrochemistry of semiconductors can be found in classical books and workshop discussions published on the subject. ${ }^{14,21,22,38,108,109}$ Theoretical interpretations of the experimental results will be presented on the basis of simple models developed initially for passive films grown on valvemetals (Al, Ta, Zr, Nb, Ti, W) and their alloys. In order to show the ability of PCS to scrutinise also complex systems we will discuss some results pertaining to passive films grown on base metals and alloys (Fe, Cr, Ni, stainless steels, Mg). Moreover recent results of a quantitative use of PCS for the chemical characterization of passive films on metallic alloys and conversion coatings will be presented. 


\section{Semiconductor/Electrolyte Junctions under Illumination: the Gärtner-Butler Model}

The modelling of photocurrent vs. potential curves at fixed irradiating wavelength (photocharacteristics) for a crystalline $\mathrm{SC} / \mathrm{El}$ junction has been carried out by several authors. ${ }^{107,111-114}$ starting from the seminal paper of Gärtner ${ }^{15}$ on the behavior of illuminated solid state Schottky barrier.

In Fig. 11 the absorption process of incident light in the bulk of a SC is sketched: $\Phi_{0}$ (in $\left.\mathrm{cm}^{-2} \mathrm{~s}^{-1}\right)$ is the photon flux entering the

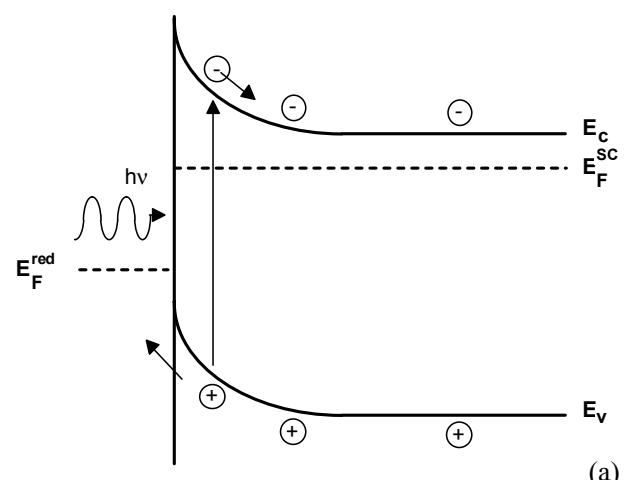

(a)

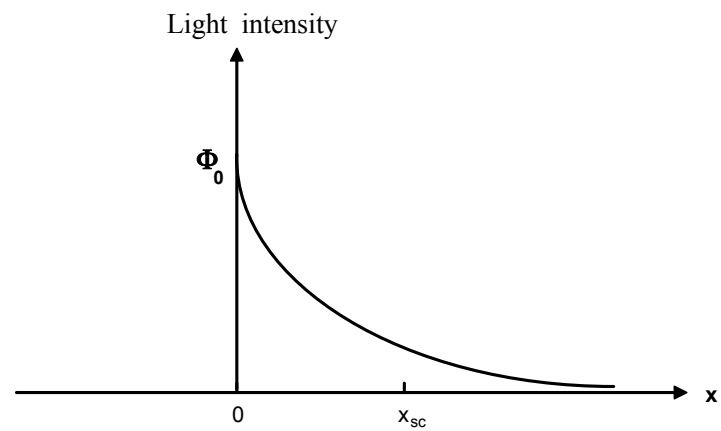

(b)

Figure 11. Schematic representation of a crystalline n-type $\mathrm{SC} / \mathrm{El}$ interface under illumination, showing the electron-hole pair generation (a) and the change of light intensity due to the absorption process within the semiconductor (b). 
semiconductor (corrected for the reflections losses at the SC/El interface), which is absorbed following the Lambert-Beer law. The number of electron-hole pairs generated per second and unit volume at any distance from the SC surface, $g(x)$, is given by:

$$
g(x)=\Phi_{0} \alpha e^{-\alpha x}
$$

where $\alpha$ (in $\mathrm{cm}^{-1}$ ), the light absorption coefficient of the semiconductor, is a function of the impinging wavelength. It is assumed that each absorbed photon, having energy $h v \geq E_{\mathrm{g}}$ originates a free electron-hole couple. In the Gärtner-Butler model the total photocurrent collected in the external circuit is calculated as sum of two terms: a migration term, $I_{\text {drift }}$, and a diffusion term, $I_{\text {diff. }}$ The first one takes into account the contribution of the minority carriers generated into the space-charge region; the second one accounts for the minority carriers entering the edge of the space-charge region from the bulk field free region $\left(x>X_{S C}\right)$ of SC. No light reflection at the rear interface is assumed, so that all the entering light is absorbed within the SC. Moreover, it is assumed that minority carriers generated in the space- charge region of the SC do not recombine at all, owing to the presence of an electric field which separates efficiently the photogenerated carriers. The same assumption is made for the minority carriers arriving at the depletion edge from the bulk region of the SC. In order to calculate $I_{\text {diff, }}$, Gärtner solved the transport equation for minority carriers, which for a n-type SC is:

$$
\frac{d p(x)}{d t}=D_{p} \frac{d^{2} p(x)}{d x^{2}}-\frac{p-p_{0}}{t}+g(x)
$$

with the boundary conditions: $p=p_{0}$ for $x \rightarrow \infty$ and $p=0$ for $x=$ $X_{S C}$. In Eq. (39), $p$ is the hole concentration under illumination, $p_{0}$ the equilibrium concentration of hole in the bulk of the (not illuminated) SC and $D_{\mathrm{p}}$ the diffusion coefficient of the holes. The zero value for $p$ at the boundary of the depletion region comes out from the previous assumption that all the holes generated into the spacecharge region are swept away without recombining. According to Gärtner, for the total photocurrent we can write: 


$$
I_{p h}=I_{d r i f t}+I_{d i f f}=e \int_{0}^{X_{s c}} g(x) d x+e D_{p}\left[\frac{d p(x)}{d x}\right]_{x=X_{s c}}
$$

where $e$ is the absolute value of the electronic charge. By solving Eq. (39) in the steady-state approximation for getting out the distribution of holes in the field free region, and by substituting Eq. (38) for $g(x)$ in the integral of the drift term, we get finally the Gärtner equation for a $\mathrm{n}$-type semiconductor: ${ }^{115}$

$$
I_{p h}=e \Phi_{0}\left[1-\frac{\exp \left(-\alpha X_{S C}\right)}{1+\alpha L_{p}}\right]+e p_{0} \frac{D_{p}}{L_{p}}
$$

where $L_{\mathrm{p}}$ is the hole diffusion length. The same equation holds for p-type SCs, with $D_{\mathrm{n}}$ and $L_{\mathrm{n}}$ (electron diffusion coefficient and diffusion length, respectively) instead of $D_{\mathrm{p}}$ and $L_{\mathrm{p}}$ and $n_{0}$ (electrons equilibrium concentration) instead of $p_{0}$.

\section{PCS Location of $U_{\mathrm{fb}}$ and $\boldsymbol{E}_{\mathrm{g}}$ Determination in c-Semiconductor/Electrolyte Junction}

For wide band gap SCs, where the concentration of minority carriers into the bulk is very small, Eq. (41) can be further simplified by neglecting the last term. In this case, by using also Eq. (5) for $X_{S C}$, Butler derived the following expression for the photocurrent at a crystalline $\mathrm{SC} / \mathrm{El}$ junction: ${ }^{107}$

$$
I_{p h}=e \Phi_{0}\left[1-\frac{\exp \left(-\alpha X_{S C}^{0} \sqrt{\left.\Delta \Phi_{S C}-\frac{k T}{e}\right)}\right.}{1+\alpha L_{p}}\right]
$$

In this equation, $X_{S C}{ }^{0}$ represents the space-charge width into the SC electrode at $1 \mathrm{~V}$ of band bending, and $\Delta \Phi_{\mathrm{SC}}=\left(U_{e}-U_{f b}\right)$. It is easy to show ${ }^{107}$ that if $\alpha X_{S C}<<1$ (slightly absorbed light) and $\alpha L_{p}<<1$ (small diffusion length for minority carriers), the photocurrent crossing the n-type $\mathrm{SC} / \mathrm{El}$ interface can be rewritten as: 


$$
I_{p h}=e \Phi_{0} \alpha X_{S C}^{0}\left(U_{e}-U_{f b}-\frac{k T}{e}\right)^{1 / 2}
$$

Equation (43) foresees a quadratic dependence of the photocurrent on the electrode potential, which can be used for getting out the flat band potential of the junction. In fact, by neglecting the term $k T / e$, a plot of $\left(I_{p h}\right)^{2}$ vs. $U_{E}$ should intercepts the voltage axis at the flat band potential, $U_{f b}$, regardless the employed $\lambda$ as long as $\alpha X_{S C}<<1$ condition is obeyed. Apart from the initial assumption of Gärtner of an ideal Schottky barrier, we have to mention that several hypotheses underlie to the use of Eqs. (41)-(43) for interpreting photoelectrochemical data. Other authors have introduced the possible existence of a kinetic control at the SC surface or within the space charge region ${ }^{111-114,116-117}$ showing that:

(a) in presence of strong surface recombination effects the onset photocurrent may occur at much higher band bending than that foreseen by the Gärtner-Butler equation; ${ }^{111}$

(b) a square root dependence of the photocurrent on the electrode potential is still compatible with the existence of some mechanism of recombination (first order kinetics) within the space charge region, ${ }^{113}$ so that the determination of the flat band potential from the square of the photocurrent vs. electrode potential plot must be taken with some caution.

According to this it is possible to write a generalized GartnerButler equation for the photocurrent at illuminated crystalline $\mathrm{SC} / \mathrm{El}$ junction as :

$$
I_{p h}=\frac{S_{t}}{S_{t}+S_{r}} e \Phi_{0} \alpha X_{s c}^{0}\left(U_{E}-U_{f b}-\frac{k T}{e}\right)^{1 / 2}
$$

In the previous equation the $S_{t} /\left(S_{t}+S_{r}\right)$ term accounts for the ratio between the minority carriers transfer reaction rate, $S_{t}$, and total recombination rate $S_{r}$. At high band bending such a ratio goes to 1 so that in presence of low recombination rate the onset photocurrent potential, $U^{*}$, could provide a close estimate of the flat band potential ${ }^{118}$. This last value is expected to be coincident or more anodic (cathodic) than $U_{f b}$ of n-type (p-type) SC/El junction. 
These aspects must be carefully considered when the photocurrent vs. potential curves are used for deriving the flat band potential of $\mathrm{SC} / \mathrm{El}$ junctions, especially in the case of corrosion layers which are far from the ideal behavior of crystalline semiconductors previously assumed. Finally we have to remark that all previous equations pertain to the steady-state values of the $d c$ photocurrent. The equations derived for steady-state remain valid also for chopped conditions provided that the lock-in measured signal remains proportional to the steady-state chopped value. ${ }^{39}$

A second important aspect embodied in Eq. (44) is the direct proportionality between the measured photocurrent and the light absorption coefficient. By considering that in the vicinity of the optical absorption threshold of the SC, ${ }^{21}$ the relationship between the absorption coefficient and optical band gap of material, $E_{g}$ can be written as:

$$
\alpha=A \frac{\left(h v-E_{g}^{o p t}\right)^{n / 2}}{h v}
$$

it is possible to derive the following expression:

$$
Q h v=\left(h v-E_{g}^{o p t}\right)^{n / 2}\left(X_{s c}^{0} \sqrt{\left|U_{e}-U_{f b}\right|}\right)
$$

$Q=\left(I_{p h}\right) / e \Phi_{0}$ represents the photocurrent collection efficiency and $E_{g}^{o p t}$ the optical threshold for the onset of photocurrent at the illuminated electrode. Eq. (46) shows that, at constant electrode potential, it is possible to get the optical band gap of the material from the dependence of the photocurrent on the wavelength (shortly referred as the photocurrent spectrum of the junction) of incident light at constant photon flux. In fact by plotting $(Q h v)^{2 / n}$ vs. hv at constant electrode potential $\left(U_{E}-U_{f b}=\right.$ const.) we get a characteristic photon energy $h v_{0}=E_{g}^{\text {opt }}$. For an ideal SC/El junction $E_{g}^{\text {opt }}$ coincides with the minimum distance in energy between the filled states of VB and empty states of CB (band gap, $E_{g}$ ) and $\mathrm{n}$ can assume different values depending on the nature of the optical transitions between states of the VB and states of the CB. 
Optical transitions at energies near the band gap of a crystalline material may be direct or indirect. In the first case no intervention of other particles is required, apart the incident photon and the electron of the VB; in the second case the optical transition is assisted by the intervention of lattice vibrations. By assuming a parabolic electronic density of states distribution, DOS, $(N(E) \propto$ $\left.E^{1 / 2}\right)$ near the band edges, in the case of direct transitions $n$ assumes values equal to 1 or 3 , depending on whether the optical transitions are allowed or forbidden in the quantum mechanical sense. ${ }^{21}$ In the case of indirect optical transitions the value of $n$ in Eq. (45) is equal to 4 . It will be shown in the case of amorphous materials that the measured optical band gap does not necessarily coincides with the band gap of crystalline material but it can be still related to the material composition and morphology.

\section{Passive Film/Electrolyte Interface under Illumination}

On going from crystalline thick SC electrodes to very thin insulating or semiconducting corrosion films on metals, new experimental features are observed which require the extension of previous interpretative models and the introduction of new theoretical concepts in order to account for novel results not observed for crystalline bulk materials. In the following we discuss the main differences in the optical properties of amorphous and crystalline materials $^{79-82}$ and how they can affect the photoelectrochemical behavior and band gap values of passive films.

\section{(i) Electronic Properties of Disordered Passive Films}

The electronic properties of disordered passive films described in previous Sections affect the generation and transport process of photocarriers and then the photoelectrochemical answer of the a$\mathrm{SC} / \mathrm{El}$ junction. The main differences in the photocurrent response of disordered thin films with respect to the case of bulk crystalline semiconductors arise from the following facts:

(a) the optical band gap of an amorphous material may coincide or not with that of the crystalline counterpart, depending on the presence of different types of defects which can modify the DOS distribution; 
(b) at variance with crystalline materials, the generation process of free carriers by the absorption of photons having energy equal or higher than the optical band gap of the film may depend on the electric field, owing to the presence of initial (geminate) recombination effects;

(c) the small thickness of passive film makes possible the optical excitation at the inner metal/film interface. This allows injecting photocarriers from the underlying metal into the VB or CB of thick film (internal photoemission), or directly into the electrolyte (external photoemission) in the case of very thin films (1-2 nm thick);

(d) the presence of reflecting metal/film and film/electrolyte interfaces makes possible the onset of multiple reflections, even for photons having energy higher than the optical absorption threshold. This fact originates interference effects in the photocurrent vs. film thickness curves. This last aspect will be not treated here for brevity; but interested readers can make use of previous published works. ${ }^{39-40}$ In the following we derive an equation for the photocurrent in amorphous $\mathrm{SC}$ and insulators by taking into account the influence of the amorphous nature on the electronic properties of materials.

\section{(ii) Amorphous Film/Electrolyte Junction under Illumination}

Due to the low mobility of carriers in amorphous materials it is reasonable to assume that a negligible contribution to the measured photocurrent arises from the field free region of the semiconductor. In this case it is quite easy to derive an expression for the migration term in the space-charge region of the a-SC, in a quite similar way to that followed by Gärtner but introducing also a recombination probability for the photocarriers generated in the space charge region of the a-SC.

Like in the Butler model, we will assume the absence of kinetic control in the solution and a negligible recombination rate at surface of the semiconductor. The limits of validity of such assumption have been discussed previously for the case of crystalline $\mathrm{SC} / \mathrm{El}$ junctions ${ }^{112}$ and they will not be repeated here. We will assume also an efficiency of free carrier generation, $\eta_{g}$, position 
independent under illumination with light having energy higher than the SC mobility gap.

Under steady-state conditions the recombination of photogenerated carriers in the space-charge region can be taken into account by assuming that the probability of any carrier photogenerated at a position $\mathrm{x}$ to leave the space-charge region is given by: ${ }^{119}$

$$
P(x, \bar{F})=\exp \left(-\frac{x}{L_{d}}\right)
$$

where $\bar{F}$ is the mean electric field in the space-charge region of the a-SC and $\mathrm{L}_{\mathrm{d}}$ is the drift length of the photocarriers ensemble in the average field approximation, given by:

$$
L_{d}=\mu \tau \bar{F}
$$

$\mu$ and $\tau$ being the drift mobility and the lifetime of the photocarriers, respectively. According to these equations and to the assumptions made, we can write:

$$
I_{p h}=I_{d r i f t}=\eta_{g} e \Phi_{0} \int_{0}^{X_{s c}} \alpha \exp (-\alpha x) P(x, \bar{F}) d x
$$

where $\Phi_{0}$ is the photon flux corrected for the reflection at the electrolyte/film interface, having assumed negligible reflections at the film/metal interface, and $\eta_{g}$, the efficiency of free carriers generation in presence of geminate recombination effects, is a function of the thermalization distance, $r_{0}$, and of $\bar{F}$. By integration of Eq. (49) we get: ${ }^{19}$

$$
I_{p h}=\eta_{g} e \Phi_{0} \frac{\alpha L_{d}}{1+\alpha L_{d}}\left[1-\exp \left(-X_{s c} \frac{1+\alpha L_{d}}{L_{d}}\right)\right]
$$




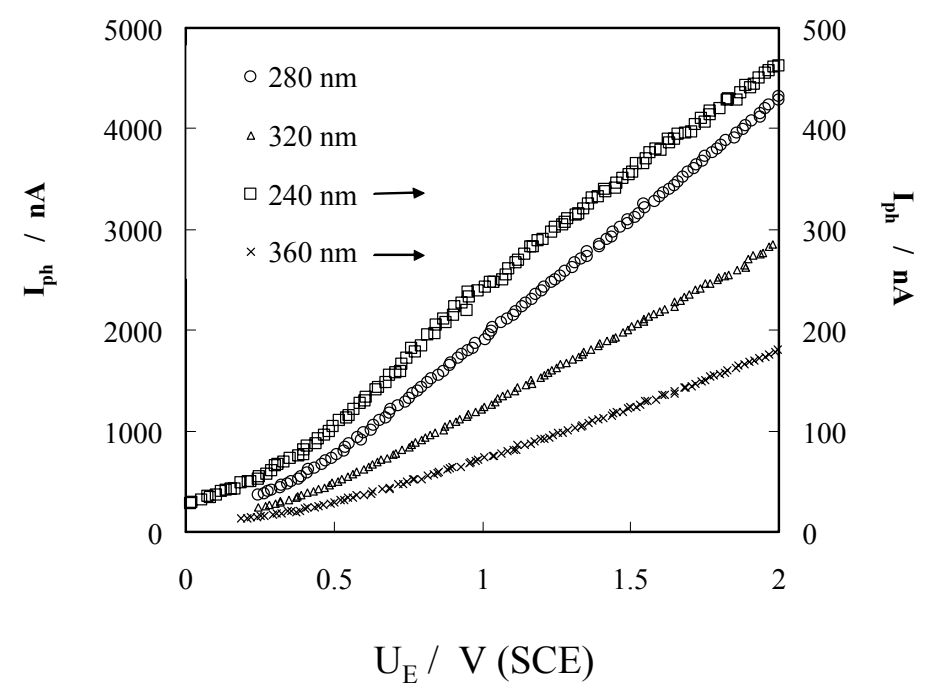

Figure 12. Photocurrent vs. potential curves at different $\lambda$ relating to an anodic films grown to $4 \mathrm{~V}(\mathrm{SCE})$ on sputter-deposited Ti-10at.\% $\mathrm{Zr}$ in $0.5 \mathrm{M} \mathrm{H}_{2} \mathrm{SO}_{4}$. $v_{\text {scan }}$ $=10 \mathrm{mV} \mathrm{s}^{-1}$ and solution: $0.5 \mathrm{M} \mathrm{H}_{2} \mathrm{SO}_{4}$.

An expression for the efficiency of free carriers generation, $\eta_{g}\left(r_{0}, \bar{F}\right)$, for amorphous materials was given by Pai and Enck. ${ }^{120}$ This last expression shows that very low efficiency of free carriers generation are expected at low electrical fields and thermalization lengths $r_{0}$ i.e., at photon energy near the mobility gap. ${ }^{121}$ This finding could affect in some extent the measured optical band gap value of amorphous anodic films.

According to Eq. (50) any dependence of $I_{\mathrm{ph}}$ vs. $U_{E}$ curves (photocharacteristics) from the energy of incident photons must be traced out to the dependence on energy and electric fields, and it has been frequently observed in amorphous SC (refs), as shown in Fig. 12 where we report the experimental photocurrent vs. potential curves at different $\lambda$ relating to an anodic films grown to 4 $\mathrm{V}(\mathrm{SCE})$ on sputter-deposited 90Ti-10Zr. ${ }^{122}$

We like to stress two aspects in the expression of the photocurrent in a-SC/El junction. The first one is that from Eq. (50) it follows a direct proportionality between the photocurrent and the 


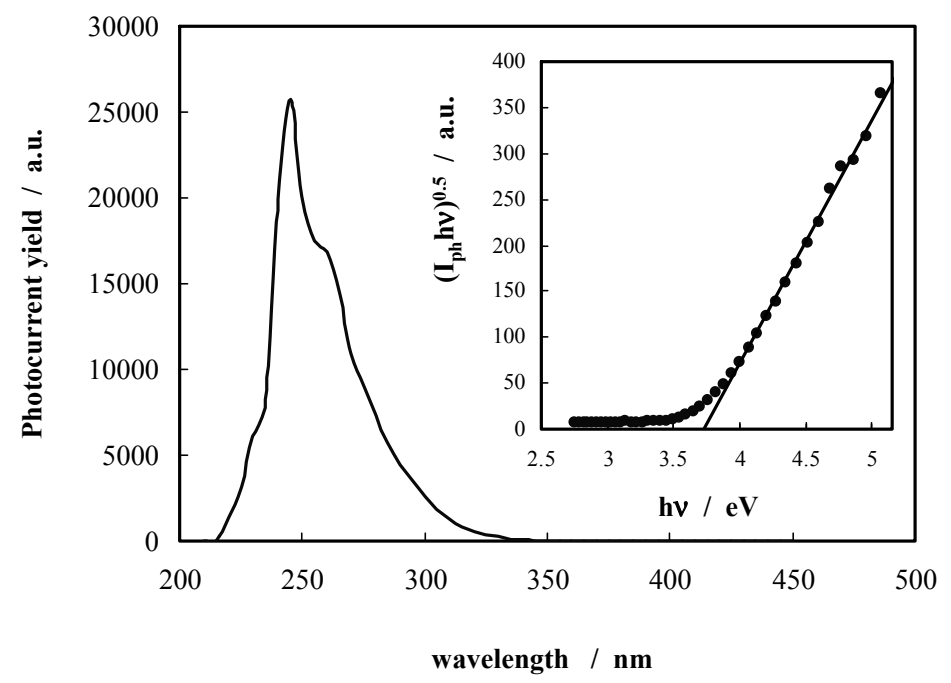

Figure 13. Photocurrent action spectrum recorded at $+2 \mathrm{~V}(\mathrm{SCE})$ for a film grown up to $4 \mathrm{~V}$ (SCE) on sputter-deposited Ti-42at.\% $\mathrm{Zr}$ in $0.1 \mathrm{M}$ ammonium pentaborate electrolyte. Inset: determination of the optical band gap by assuming indirect transitions. Reprinted from M. Santamaria, F. Di Quarto, and H. Habazaki, "Photocurrent spectroscopy applied to the characterization of passive films on sputter-deposited Ti-Zr alloys." Corr. Sci. 50 (2008) 2012, Copyright (2008) with permission from Elsevier.

absorption light coefficient for $\alpha L_{d}>>1$ (no recombination) and $\alpha X_{S C}<<1$ (slightly absorbed light), as previously derived for crystalline materials (see Eq. 43). On the other hand for $\alpha L_{d}<<1$ still a direct proportionality between $I_{p h}$ and $\alpha$ is assured by the fractional term $\alpha L_{d} /\left(1+\alpha L_{d}\right)$. According to these considerations, we can still assume for amorphous SCs a direct proportionality between the photocurrent yield, $Q=I_{p h} / e \Phi_{0}$, and the light absorption coefficient, $\alpha$, in the vicinity of the absorption edge under constant electrode potential. Like for crystalline materials, this allows to replace $\alpha$ with the photocurrent yield in deriving the optical band gap of amorphous semiconducting films from the photocurrent spectra (see Fig. 13).

A second aspect we like to stress is that Eq. (50) contains as a particular case the expression of the photocurrent for an amor- 


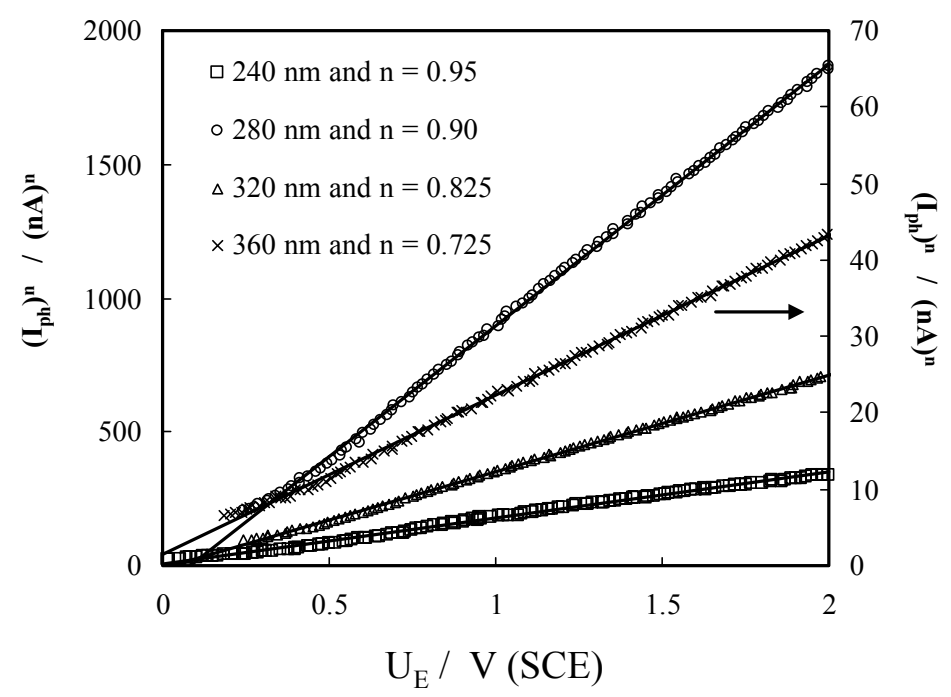

Figure 14. Fitting of the photocurrent vs. potential curves of Figure 12.

phous insulator/electrolyte junction after substitution of the film length, $d_{f}$, to the space charge region $X_{S C}$. According to this we can write for insulating film/electrolyte junction the relationship:

$$
I_{p h}=e \Phi_{0} \eta_{g} \frac{\alpha L_{d}}{1+\alpha L_{d}}\left[1-\exp \left(-d_{f} \frac{1+\alpha L_{d}}{L_{d}}\right)\right]
$$

The usual expression for the mean electric field in insulator $F_{a v}=$ $\left(U_{E}-U_{f b}\right) / d_{f}$ holds in absence of trapping phenomena. ${ }^{39,123}$ In both cases the variation of the efficiency of generation with the electric field and photons energy, through $r_{0}$, can account for changes in $E_{g}^{o p t}$ values measured at different electrode potentials as well as for the dependence from the incident photon energy of the photocurrent vs. potential curves. This last aspect has been deeply discussed both for semiconducting $\left(\mathrm{a}-\mathrm{WO}_{3}\right.$ and $\left.\mathrm{a}-\mathrm{TiO}_{2}\right)$ and insulating $\left(\mathrm{a}-\mathrm{Ta}_{2} \mathrm{O}_{5}\right)$ anodic films in previous works. ${ }^{39,121,124}$ In Fig. 14 we report the fitting curves of experimental data for films grown to 4 $\mathrm{V}(\mathrm{SCE})$ on $90 \mathrm{Ti}-10 \mathrm{Zr}$. The details of fitting procedure can be 
found in Ref. [122]; here we have to mention that the flat band potential can now be derived as a fitting parameter of the photo characteristics.

\section{(iii) Optical Gap in Amorphous Materials}

It was previously mentioned that optical transitions at energies near the band gap of a crystalline material may be direct (allowed or forbidden) or indirect. In the case of amorphous materials, owing to the relaxation of the k-conservation selection rule:

"no intervention of phonons is invoked to conserve momentum and all energy required is provided by the incident photons." 81

By assuming again a parabolic DOS distribution in the vicinity of the mobility edges of both the conduction and valence band (above $E_{C}$ and below $E_{V}$, with reference to Fig. 3) it has been shown $^{82}$ that for amorphous materials the following relationship holds:

$$
\alpha h v=\text { const }\left(h v-E_{g}^{m}\right)^{2}
$$

where $E_{g}^{m}=E_{C}-E_{V}$ is now the mobility gap of the a-SC (see Fig.

$3)$. The exponent 2 is reminiscent of the indirect optical transitions in crystalline material but now photons interact with the solid as a whole: this type of transition in amorphous materials is termed non-direct. Because some tailing of states is theoretically foreseen for a-SC by any proposed model of DOS, $E_{g}^{m}$ represents an extrapolated rather then a real zero in the density of states. On the other hand in presence of a DOS distribution varying linearly with energy in the ranges $E_{C}-E_{A}$ and $E_{B}-\boldsymbol{E}_{\boldsymbol{V}}$ of Fig. $3 \mathrm{~b}$, it is possible to get for the absorption coefficient ${ }^{81}$ of amorphous material the following relationship:

$$
\alpha h v=\operatorname{const}\left(h v-E_{g}^{o p t}\right)^{2}
$$


where $E_{g}^{\text {opt }}$ now represents the difference of energy $\left(E_{A}-E_{V}\right)$ or $\left(E_{C}-E_{B}\right)$ in Fig. 3b, whichever is smaller, whilst the constant assumes values close to $10^{5} \mathrm{eV}^{-1} \mathrm{~cm}^{-1}$. The range of energy in which Eq. (53) should be valid is in the order of $0.4 \mathrm{eV}$ or less. ${ }^{81}$ In order to distinguish between these two different models of optical transitions, both giving a similar dependence of absorption coefficient on the photon energy, we will refer to the first one as the Tauc's approximation and to the second one as the Mott-Davis approximation. From the first one we derive an estimation of the mobility gap and from the second one the optical gap of amorphous materials. If $(\alpha h v)^{0.5}$ vs. hv plots display a linear region larger than 0.4 $\mathrm{eV}$ it seems more correct to interpret the data on the basis of the Tauc's model of optical transitions. The coexistence of both types of transitions has been reported for thin anodic films grown on niobium, ${ }^{125}$ with the presence of a mobility gap in the order of 3.5 $\mathrm{eV}$ in the high photon energy range extending around $1 \mathrm{eV}$ and an optical gap (in the Mott's sense) of about $3.05 \mathrm{eV}$.

In the case of anodic films on valve-metals, an exponential decrease in the photocurrent yield (Urbach tail) as a function of photon energy is frequently observed at photon energies lower than the mobility gap. A possible origin of such dependence can be attributed to a variation of the light absorption coefficient according to the following law:

$$
\alpha=\alpha_{0} \exp \left(-\gamma \frac{E_{0}-h v}{k t}\right)
$$

with $\gamma$ and $\alpha_{0}$ constant. This relationship, which has been found to hold also for crystalline materials, has been rationalized in the case of a-SCs by assuming an exponential distribution of localized states in the band edge tails. ${ }^{126}$ In this case $E_{0}$ marks the energy where $\ln \alpha v s$ hv (Urbach plot) ceases to be linear. This value frequently coincides with the mobility gap value determined according to Eq. (52).

A typical example is reported in Fig. 15 for the anodic film of Fig. 13: the value of $E_{g}^{m}$, equal to about $3.75 \mathrm{eV}$, is in good agreement with the value of $E_{0}(\cong 3.85 \mathrm{eV})$ derived form the Ur- 


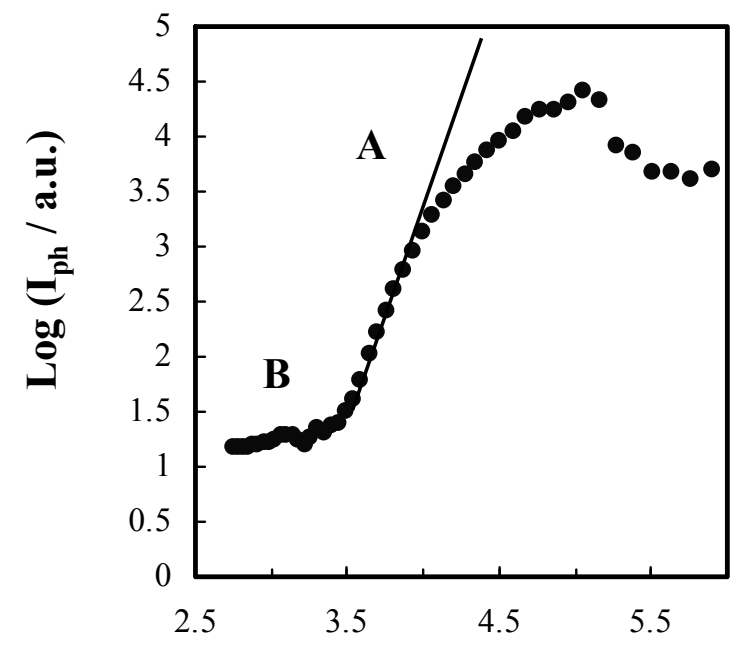

hv / eV

Figure 15. Urbach plot relating to the anodic film of Figure 13. Reprinted from M. Santamaria, F. Di Quarto, and H. Habazaki, "Photocurrent spectroscopy applied to the characterization of passive films on sputter-deposited Ti-Zr alloys." Corr. Sci. 50 (2008) 2012, Copyright (2008) with permission from Elsevier.

bach plot. Other explanations have been suggested for this behavior in the case of crystalline materials. ${ }^{81}$ At very low absorption levels, a second exponential part in the Urbach plot (weak absorption tail, B in Fig. 15c) can be frequently observed. Such behavior has been interpreted by assuming a dependence of the light absorption coefficient on energy according to the following relationship:

$$
\alpha \propto \exp \left(\frac{h v}{E_{t}}\right)
$$

where the energy parameter $E_{t}$ is always larger than $E_{0}{ }^{127}$ This part of the $\log \alpha$ vs. $h v$ plot is not as well reproducible as that at higher 
energy due the high structure sensitivity of the light absorption coefficient in this energy range. ${ }^{127}$

In agreement with a general statement reported in Ref. [81], it has been suggested ${ }^{39-40}$ that, in absence of appreciable differences in short range order of amorphous and crystalline counterparts, the mobility gap of amorphous anodic films should be equal or larger than the band gap of the crystalline counterpart. Such a difference in optical band gap value can be assumed as a measure of the influence of lattice disorder on optical gap of the films.

In Table 1 we report the mobility gap and band gap values of some passive films grown on valve metals. The difference $\Delta E_{a m}=\left(E_{g}^{m}-E_{g}^{c r y s t}\right)$ in the range of $0.1-0.35 \mathrm{eV}$, is in agreement with the expected extension of the localized states regions near the band edges due to the lattice disorder. ${ }^{81}$ Values of $E_{0}$ (see

Eq. 54) nearly coincident with the mobility gap, $E_{g}^{m}$, have been

Table 1

Measured Optical Gap, $E_{g}^{m}$ for Passive Films on Pure Metals Compared with the Band Gap of the Crystalline Counterpart, $E_{g}^{c r y s t} \cdot \Delta E_{a m}$ is the Difference Between $E_{g}^{m}$ and $E_{g}^{c r y s t}$.

\begin{tabular}{|c|c|c|c|}
\hline Phase & $\begin{array}{c}E_{g}^{m} \\
(\mathrm{eV})\end{array}$ & $\begin{array}{c}E_{g}^{c r y s t} \\
(\mathrm{eV})\end{array}$ & $\begin{array}{l}\Delta E_{a m} \\
(\mathrm{eV})\end{array}$ \\
\hline $\begin{array}{l}\mathrm{ZrO}_{2} \\
\mathrm{Ta}_{2} \mathrm{O}_{5} \\
\mathrm{Nb}_{2} \mathrm{O}_{5}\end{array}$ & $\begin{array}{l}4.70 \sim 4.80^{137} \\
3.95 \sim 4.05^{137} \\
3.30 \sim 3.40^{137}\end{array}$ & $\begin{array}{l}4.50^{\mathrm{a}} \\
3.85^{\mathrm{a}} \\
3.15^{\mathrm{a}}\end{array}$ & $\begin{array}{l}0.20 \sim 0.30 \\
0.10 \sim 0.20 \\
0.15 \sim 0.25\end{array}$ \\
\hline $\mathrm{TiO}_{2}$ & $3.20 \sim 3.35^{\mathrm{a}}$ & $\begin{array}{l}3.05 \text { (rutile) }^{\mathrm{a}} \\
3.20 \text { (anatase) }^{\mathrm{a}}\end{array}$ & $0.15 \sim 0.20$ \\
\hline $\begin{array}{l}\mathrm{WO}_{3} \\
\mathrm{MoO}_{3}\end{array}$ & $\begin{array}{l}2.95 \sim 3.15^{137} \\
2.95 \sim 3.10^{137}\end{array}$ & $\begin{array}{l}2.75^{137} \\
2.90^{144}\end{array}$ & $\begin{array}{l}0.20 \sim 0.40 \\
0.05 \sim 0.20\end{array}$ \\
\hline $\begin{array}{l}\mathrm{Cr}_{2} \mathrm{O}_{3} \\
\mathrm{NiO} \\
\mathrm{Cu}_{2} \mathrm{O}\end{array}$ & $\begin{array}{l}3.30 \sim 3.55^{137} \\
3.43^{137} \\
1.86^{137}\end{array}$ & $\begin{array}{l}3.30^{137} \\
3.45 \sim 3.55^{137} \\
1.86^{137}\end{array}$ & $\begin{array}{l}0.0 \sim 0.25 \\
0 \\
0\end{array}$ \\
\hline $\begin{array}{l}\mathrm{Fe}_{2} \mathrm{O}_{3} \\
\mathrm{Fe}_{0.25} \mathrm{Ti}_{0.75} \mathrm{O}_{1.875} \\
\mathrm{Fe}_{0.1} \mathrm{Ti}_{0.9} \mathrm{O}_{1.95}\end{array}$ & $\begin{array}{l}1.90 \sim 1.95^{137} \\
2.95^{137} \\
3.15^{145}\end{array}$ & $\begin{array}{l}1.90^{137} \\
2.80^{a} \\
3.00^{a}\end{array}$ & $\begin{array}{l}0 \sim 0.05 \\
0.15 \\
0.15\end{array}$ \\
\hline
\end{tabular}


frequently derived for passive films on valve metals. It seems quite reasonable to suggest, for such a class of amorphous materials, a band model similar to that shown in Fig. $3 b$ with an exponentially decreasing DOS in the mobility gap of the films at energies lying below $E_{C}$ and/or above $E_{V}$. A mobility gap of passive film lower than the band gap of the crystalline counterpart must be interpreted as an indication that differences are present in the short-range order of the two phases. A different short-range order can imply the formation of a defective structure, with a high density of localized states within the mobility gap as well as changes in the density of the passive film, which is known to affect also the value of the optical gap in amorphous materials. ${ }^{79-81}$ The experimental findings on passive films and corrosion layers suggest that large differences in optical gap values, between amorphous and crystalline counterparts, should be traced out to a different chemical environment around the metallic cation or to the presence of large amount of defects within the passive films, originating electronic states within the mobility gap. A remarkable case is reported in Ref. [128], where the incorporation of organic species into anodic films, grown on electropolished Al samples in tartrate containing solution, originated a DOS distribution within the band gap of a$\mathrm{Al}_{2} \mathrm{O}_{3}$ so allowing the onset of anodic photocurrent at photon energies $(h v \cong 3.0 \mathrm{eV})$ well below the band gap of $\mathrm{Al}_{2} \mathrm{O}_{3}\left(E_{g} \geq 6.30\right.$ $\mathrm{eV})$.

\section{(iv) Photoemission Phenomena at the Metal/Passive Film Interface}

In this Section we discuss the role of the inner metal/film interface in the generation processes of photocarriers for thin and thick passive films. In presence of thin passive films it is possible that under illumination a large fraction of photons impinging the film/solution interface arrive at the metal/film interface, by exciting metal electrons to higher energy levels and leaving vacant states below the Fermi level of the metal. The fate of the excited states into the metal depends on the occurrence of different physical deactivation processes at this interface. Apart the thermal deactivation by scattering of excited electrons with the lattice vibrations, photoemission phenomena of excited photocarriers can be observed. In the case of very thin passive films $\left(d_{o x} \leq 2 \mathrm{~nm}\right)$ exter- 
nal (into the electrolytic solution) photoemission processes become possible by tunnelling of excited electrons or holes at the metal surface throughout the film. A hole photoemission process has been suggested in the case of a gold electrode covered with a very thin oxide. ${ }^{129}$ The photoemission of electrons directly from the metal to the ground state of liquid water has been observed more frequently through very thin oxide films covering metals. ${ }^{130-132}$ When such an external photoemission process occurs, in absence of diffuse double layer effects or large adsorbed molecules, it is possible to write for the emission photocurrent the so-called five half (5/2) power law, which gives the dependence of photocurrent from photon energy and electrode potential as: ${ }^{39,40,133,134}$

$$
I_{p h}=\text { const. }\left(h v-h v_{0}-|e| U_{E}\right)^{5 / 2}
$$

where $U_{E}$ is the electrode potential measured with respect to a reference electrode, $h v_{0}$ is the photoelectric threshold at zero electrode potential (changing with reference electrode) and hv is the photon energy in eV. At constant potential the photocurrent yield can be expressed as:

$$
Q^{0.4}=\text { const. }\left(h v-E_{t h}\right)
$$

where $E_{t h}=h v_{0}+|e| U_{E}$ is the measured photoemission threshold, dependent on the imposed electrode potential. It follows that a change in the photoemission threshold vs. potential of $1 \mathrm{eV} / \mathrm{V}$ is expected. By assuming that the electron photoemission process occurs from the metal Fermi level to the bottom of the conduction band ${ }^{134}$ of solvent, $E_{\text {solvent }}^{C}$, this last level can be estimated (with respect to the vacuum) according to the equation:

$$
E_{\text {solvent }}^{C}=E_{\text {th }}-|e| U_{E}(\text { ref })-|e| U_{E, \text { ref }}(\text { vac })
$$

where $E_{t h}$ is the photocurrent emission threshold calculated according to Eq. (56b), $U_{E}$ is the electrode potential with respect to a reference electrode, and $U_{E, \text { ref }}(\mathrm{vac})$ is the reference electrode potential with respect to the vacuum. A value of $4.60 \mathrm{~V}$ has been assumed on the vacuum scale for the NHE according to different authors. ${ }^{23}$ 


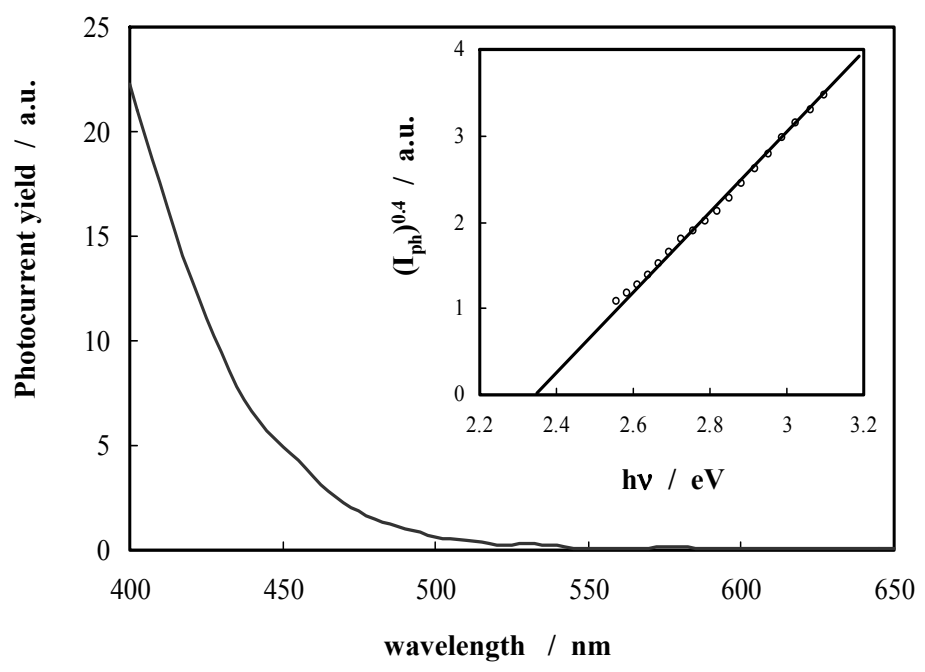

Figure 16. Photocurrent spectrum relating to $\mathrm{Mg}$ after mechanical treatment recorded by polarizing the electrode at $-1.94 \mathrm{~V}$ (MSE) in $0.1 \mathrm{M} \mathrm{Na}_{2} \mathrm{SO}_{4}(\mathrm{pH}=$ 6.15). Inset: estimate of the external photoemission threshold. Reprinted from M. Santamaria, F. Di Quarto, S. Zanna, and P. Marcus, "Initial surface film on magnesium metal: A characterization by X-ray photoelectron spectroscopy (XPS) and photocurrent spectroscopy (PCS)." Electrochim. Acta 53 (2008) 1314, Copyright (2007) with permission from Elsevier.

Figure 16 shows the photocurrent spectrum, in the long wavelengths region, recorded at constant potential $U_{E}=-1.94 \mathrm{~V}$ (MSE) (more cathodic than the respective $U_{O C}$ potential), for a freshly prepared mechanically cleaned $\mathrm{Mg}$ electrode (MTE) at $\mathrm{pH}=6.15$, from which an external photoemission threshold of $2.35 \mathrm{eV}$ was estimated (see inset) ${ }^{135}$. At very close electrode potential $(-1.97 \leq$ $\left.U_{E} \leq-1.84 \mathrm{~V}(\mathrm{MSE})\right)$ an almost identical photoemission threshold was measured for MTE Mg electrodes in a wide range of solution $\mathrm{pH}(6<\mathrm{pH}<14)$, allowing to estimate an almost constant $E_{H 2 O}^{C} \cong$ $-1 \mathrm{eV}$ (see Fig. 17). This value agrees quite well with previously reported value $(-1 \pm 0.1 \mathrm{eV}) .^{39,40,133,136}$

The same photoemission processes can operate in organic solvent, as shown in Fig. 18, where we report the cathodic photocurrent spectra of MTE Mg electrode recorded at - 2.4 V(SCE) in 0.1 


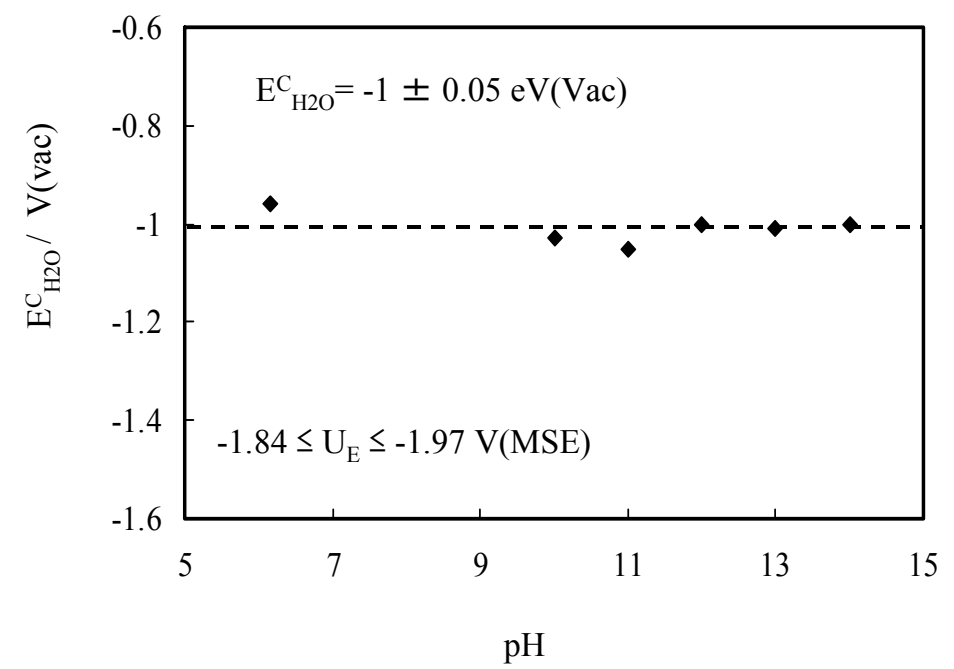

Figure 17. Estimated energy levels of water conduction band from external photoemission data relating to $\mathrm{Mg}$ after mechanical treatment as a function of solution pH. Reprinted from M. Santamaria, F. Di Quarto, S. Zanna, and P. Marcus, "Initial surface film on magnesium metal: A characterization by X-ray photoelectron spectroscopy (XPS) and photocurrent spectroscopy (PCS)." Electrochim. Acta 53 (2008) 1314, Copyright (2007) with permission from Elsevier.

$\mathrm{M} \mathrm{LiClO}_{4}$ in propylene carbonate electrolyte. As evidenced in the figures (see inset) the photocurrent yield plotted according to the $5 / 2$ law (see Eq. 56) gives an onset photocurrent threshold value of $\sim 1.8 \mathrm{eV}$. The threshold values are reported as a function of $U_{E}$ in Fig. 19. A slope of $\sim 1 \mathrm{eV} / \mathrm{V}$ was derived, in agreement with the theoretical expectation. ${ }^{134}$ It is interesting to mention that the $\left(I_{p h}\right)^{0.4}$ vs. $U_{\mathrm{E}}$ plots at different wavelengths $(\lambda=450,400 \mathrm{~nm})$ (see Fig. 20) showed a zero photocurrent potential value which shifted with photon energy (about $1 \mathrm{~V} / \mathrm{eV}$ ) as foreseen from the theory (see Eq. 56). ${ }^{134}$

The data of Fig. 19 and 20 allow to locate the bottom of the conduction band of the organic solvent (or the energy level of photoemitted electron in propylene carbonate) by subtracting to the photoemission threshold $(4.15 \mathrm{eV})$, at zero electrode potential, the Fermi energy level of SCE measured with respect to the vacuum $(-4.84 \mathrm{eV})$. It came out that the bottom of the conduction band of 


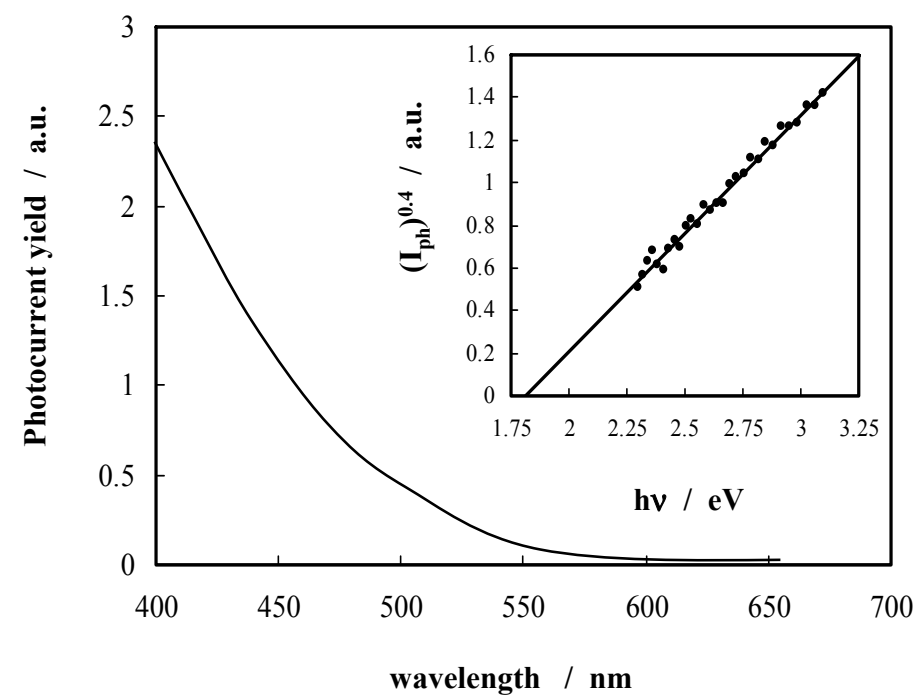

Figure 18. Photocurrent spectrum relating to $\mathrm{Mg}$ after mechanical treatment recorded by polarizing the electrode at $-2.40 \mathrm{~V}(\mathrm{SCE})$ in $0.1 \mathrm{M} \mathrm{LiClO}_{4}$ propylene carbonate electrolyte. Inset: estimate of the external photoemission threshold. Reprinted from M. Santamaria, F. Di Quarto, S. Zanna, and P. Marcus, "Initial surface film on magnesium metal: A characterization by X-ray photoelectron spectroscopy (XPS) and photocurrent spectroscopy (PCS)." Electrochim. Acta 53 (2008) 1314, Copyright (2007) with permission from Elsevier.

propylene carbonate is located at about $-0.7 \mathrm{eV}$ with respect to the vacuum. Such a value incorporates an uncertainty on the potential drop at the organic solvent/SCE junction. However the value derived for the conduction band bottom edge of propylene carbonate compares quite well with corresponding values reported in Ref. [134] for other organic solvents and it allows to get an estimate for the Volta potential difference $(\approx-0.3 \mathrm{~V})$ between $\mathrm{Mg}$ and propylene carbonate at zero charge.

In the case of thicker films $\left(d_{O x} \geq 5 \mathrm{~nm}\right)$, where the external photoemission processes are forbidden, the possibility of an internal photoemission process due to the injection of photoexcited electrons (or holes) from the metal into the $\mathrm{CB}$ (or VB) of the passive film must be considered. In such a case the internal photocur- 


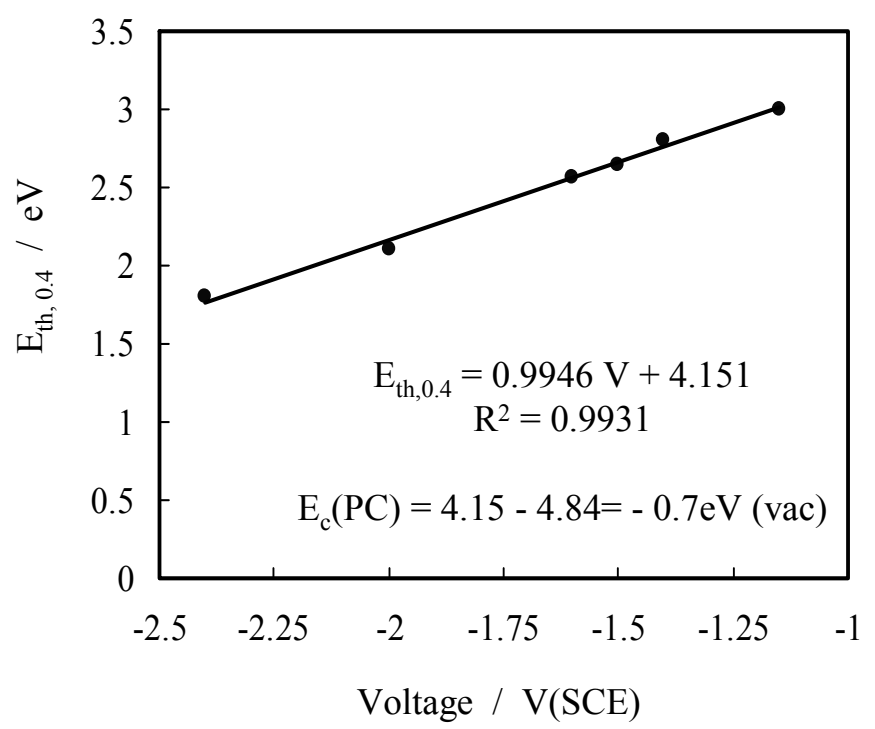

Figure 19. External photoemission threshold as a function of electrode potential relating to $\mathrm{Mg}$ after mechanical treatment. Sol: $0.1 \mathrm{M} \mathrm{LiClO}_{4}$ in propylene carbonate. Reprinted from M. Santamaria, F. Di Quarto, S. Zanna, and P. Marcus, "Initial surface film on magnesium metal: A characterization by X-ray photoelectron spectroscopy (XPS) and photocurrent spectroscopy (PCS)." Electrochim. Acta 53 (2008) 1314, Copyright (2007) with permission from Elsevier.

rent emission varies with the photon energy according to the socalled Fowler photoemission law: ${ }^{134}$

$$
Q=\text { const }\left(h v-E_{t h}\right)^{2}
$$

where $E_{t h}$ is the internal photoemission threshold energy, which can be obtained from a plot of $Q Q^{0.5}$ vs. the photon energy, hv, at constant photon flux. This threshold is a measure of the distance in energy between the Fermi level of the metal and the CB (electron photoemission) or VB (hole photoemission) edge of the film. The 


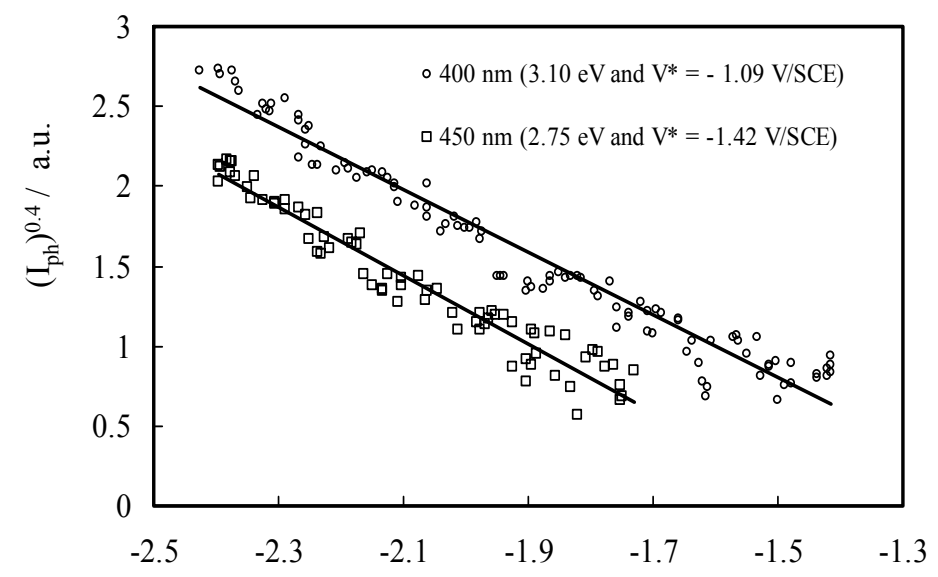

Voltage / V(SCE)

Figure 20. $\left(I_{p h}\right)^{0.4}$ vs. $U_{E}$ plots for mechanical treated $\mathrm{Mg}$, recorded by scanning the electrode potential at $10 \mathrm{mV} \mathrm{s}^{-1}$. Sol: $0.1 \mathrm{M} \mathrm{LiClO}_{4}$ in propylene carbonate. Reprinted from M. Santamaria, F. Di Quarto, S. Zanna, and P. Marcus, "Initial surface film on magnesium metal: A characterization by X-ray photoelectron spectroscopy (XPS) and photocurrent spectroscopy (PCS)." Electrochim. Acta 53 (2008) 1314, Copyright (2007) with permission from Elsevier.

occurrence of electron or hole internal photoemission in the case of insulating films is established by the direction of the electric field, and in turn by the electrode potential value with respect to the photocurrent sign inversion potential. In absence of trapping effects, the inversion photocurrent potential can be used to determine the flat band potential of insulating passive films. In the case of insulating anodic films on valve-metals internal electron photoemission processes are usually observed under cathodic polarization and under illumination with photons having energy lower than the optical band gap of the film. ${ }^{130,132}$ In Fig. 21 we report the determination of the internal photoemission threshold for a passive film grown on $\mathrm{Mg}$ metal after mechanical treatment and immersion in $0.1 \mathrm{M} \mathrm{NaOH}$ at $80^{\circ} \mathrm{C}$ for $1 \mathrm{~h}$, recorded by polarizing the electrode at $-1.53 \mathrm{~V}(\mathrm{MSE})$ in $0.1 \mathrm{M} \mathrm{NaOH}$ at room temperature. 


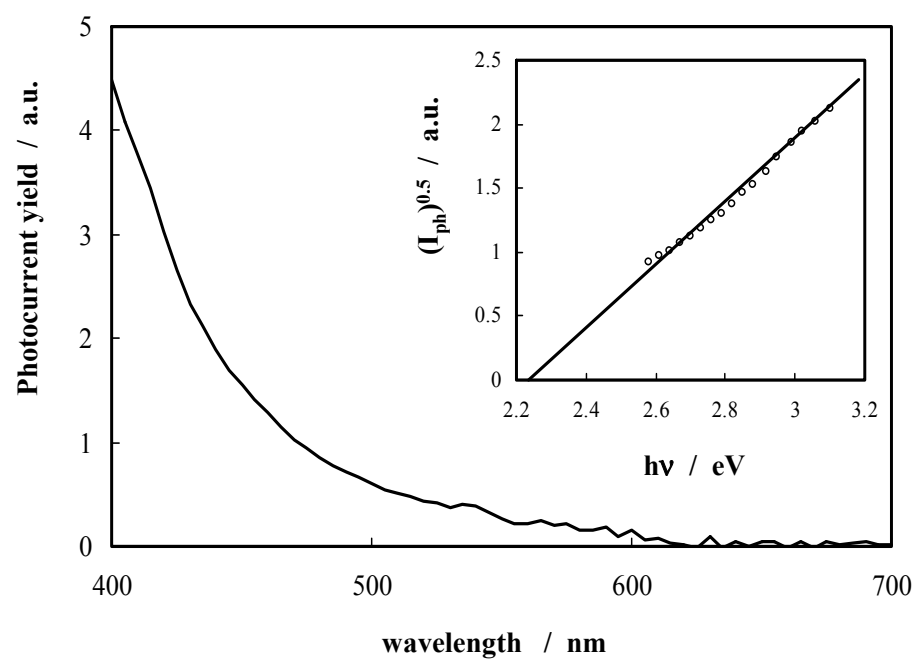

Figure 21. Photocurrent spectrum relating to $\mathrm{Mg}$ after mechanical treatment and immersion in $0.1 \mathrm{M} \mathrm{NaOH}$ at $80^{\circ} \mathrm{C}$ for $1 \mathrm{~h}$, recorded by polarizing the electrode at $-1.93 \mathrm{~V}(\mathrm{MSE})$ in $0.1 \mathrm{M} \mathrm{NaOH}$ at room temperature. Inset: estimate of the Fowler photoemission threshold. Reprinted from M. Santamaria, F. Di Quarto, S. Zanna, and P. Marcus, "Initial surface film on magnesium metal: A characterization by X-ray photoelectron spectroscopy (XPS) and photocurrent spectroscopy (PCS)." Electrochim. Acta 53 (2008) 1314, Copyright (2007) with permission from Elsevier.

It is interesting to stress that the thickening of the film grown in alkaline solution at high temperature hinders the direct photoemission process of electrons into the electrolyte observed for the same metal after scraping. In the case of semiconducting films no evidence of internal photoemission process is expected, owing to the absence of any electric field at the metal/film interface, as long as the space charge region of the SC is less than the film thickness. The knowledge of the internal photoemission thresholds allow to locate the energy level of the conduction band of the oxide films with respect to the Fermi level of the underlying metal, once the work function of the metal is known. In Table 2 the internal thresholds for cathodic photoemission of a series of insulating oxide films grown on different valve-metals have been reported. 
Table 2

Threshold Energy Values Derived from the Fowler Plots for the Internal Photoemission Process at the Metal/Passive Film Interface. The Uncertainty is of $\pm 0.1 \mathrm{eV}$.

\begin{tabular}{lc}
\hline Interface & $E_{t h}^{F}$ \\
& $(\mathrm{eV})$ \\
\hline $\mathrm{Y} / \mathrm{Y}_{2} \mathrm{O}_{3}$ & $2.10^{39}$ \\
$\mathrm{Bi} / \mathrm{Bi}_{2} \mathrm{O}_{3}$ & $1.40^{39}$ \\
$\mathrm{~Pb} / \mathrm{PbO}$ & $1.00^{39}$ \\
$\mathrm{Zr} / \mathrm{ZrO}_{2}$ & $1.80^{39,146}$ \\
$\mathrm{Hf} / \mathrm{HfO}_{2}$ & $2.15^{39,147}$ \\
$\mathrm{Al} / \mathrm{Al}_{2} \mathrm{O}_{3}$ & $2.00^{39}$ \\
$\mathrm{Ta} / \mathrm{Ta} \mathrm{O}_{5}$ & $1.50^{39}$ \\
$\mathrm{Mg} / \mathrm{Mg}(\mathrm{OH})_{2}$ & $2.15^{135}$ \\
anodic oxide on Mo-79at.\%Ta & $1.75^{39,144}$ \\
anodic oxide on Al-34at.\%Ta & $1.53^{39,143}$ \\
anodic oxide on Al- $25 \mathrm{at} \% \mathrm{Nb}$ & $2.25^{a}$ \\
anodic oxide on Al- 44at.\%Hf $\% \mathrm{Hf}$ & $2.40^{a}$ \\
anodic oxide on Al- 34at.\%Hf & $2.48^{a}$ \\
\hline${ }^{a}$ Unpublished data. &
\end{tabular}

${ }^{a}$ Unpublished data.

\section{Band Gap and Oxide Film Composition}

\section{(i) Binary Crystalline Oxides}

In spite of the relevant information on the structure of the passive film/electrolyte junctions obtainable by PCS a more widespread use of this technique in corrosion studies has been hampered by the lack of a viable theory which relates the measured $E_{g}$ values to the passive film composition. In general terms such a task is a very challenge one, also for advanced theoretical methods based on quantum mechanical calculations. It is within the aims of this chapter to show that it is possible to relate the band gap values of numerous oxides to their composition by means of a semiempirical approach. In a previous work ${ }^{137}$ it has been shown that such a task could be accomplished by proposing the following general correlation between the band gap of crystalline oxides and the difference of electronegativity of their constituents:

$$
E_{g}=2\left[E_{I}\left(\chi_{M}-\chi_{O}\right)^{2}+\Xi\right]
$$


where according to Phillips ${ }^{138} E_{I}$ is the extra-ionic energy unit orbitally dependent, assumed "to vary with hybridisation configuration, i.e., with different atomic co-ordination in different crystal structures." $\chi_{M}$ and $\chi_{O}$ are the electronegativities of metal and oxygen respectively. By plotting the band gap values of numerous oxides as a function of $\left(\chi_{M}-\chi_{O}\right)^{2}$ it comes out that the proposed correlation was able to provide:

(a) apart few exceptions (see below), two clearly separated interpolating lines were found for sp-metal and d-metal oxides, according to the following equations:

$$
\begin{array}{ll}
\text { d-metal oxide: } & E_{g}=1.35\left(\chi_{M}-\chi_{O}\right)^{2}-1.49 \text { in eV }(60 \mathrm{a}) \\
\text { sp-metal oxide: } & E_{g}=2.17\left(\chi_{M}-\chi_{O}\right)^{2}-2.71 \text { in eV }(60 \mathrm{~b})
\end{array}
$$

(b) a better fitting of the experimental data with respect to previous proposed correlation as evidenced by the higher correlation coefficient values.

From the d-metal correlation it follows that metallic oxides $\left(E_{g} \cong 0\right)$ are expected to form on metals having Pauling's electronegativity value around 2.45 in agreement with the common experience that noble metal oxides at higher oxidation states $\left(\mathrm{RuO}_{2}\right.$, $\mathrm{IrO}_{2}$ notably) usually display metallic conductivity. From a practical point of view two more aspects are interesting for corrosion studies:

(a) in this correlation $\mathrm{NiO}$ stays neatly on the sp-metal oxides interpolating line, whilst $\mathrm{Cr}_{2} \mathrm{O}_{3}, \mathrm{FeO}, \mathrm{Fe}_{2} \mathrm{O}_{3}, \mathrm{Cu}_{2} \mathrm{O}$ and $\mathrm{CuO}$ are well interpolated as d-metal oxides;

(b) three non transitional-metal oxides $\left(\mathrm{PbO}, \mathrm{In}_{2} \mathrm{O}_{3}, \mathrm{Tl}_{2} \mathrm{O}_{3}\right)$ are better interpolated like d-metal oxides. According to this an intriguing d-/sp-metal oxides dividing line along the diagonal $\mathrm{Zn}, \mathrm{In}, \mathrm{Pb} / \mathrm{Ga}, \mathrm{Sn}, \mathrm{Bi}$ appears, with some of sp-metals (In, $\mathrm{Tl}, \mathrm{Pb}$ ) of higher atomic number showing a d-like behavior in terms of optical band gap vs. $\left(\chi_{M}-\chi_{O}\right)^{2}$ correlation. Like all semiempirical approaches the correlation cannot account for such a different behavior, moreover a further complication comes out from the fact that in some ma- 
terials the nature of optical transition (direct or indirect) can affect sensibly the measured band gap value.

As for the electronegativity values in all calculations the Pauling's scale of electronegativity, ${ }^{139}$ integrated with the GordyThomas values ${ }^{140}$ has been used with the exception of Tl(III) for which the value given by Allred ${ }^{141}$ has been preferred. The electronegativity value of different elements, calculated by using the experimental band gap values of the corresponding oxides and according to the best fitting straight lines, differ from those reported in Refs. [139-141] by a quantity of about 0.05 , which is more or less the uncertainty given by the authors. According to this procedure in Table 3 the estimated electronegativity for a group of lanthanides metals, obtained by using the d-metal correlation and the band gap values reported in the same table, is reported. The electronegativity values for such a group of metals stay within the limits 1.1-1.3 usually reported for f-block elements and in fair agreement with those reported by Allred ${ }^{141}$ for the same elements at different oxidation states. Although the proposed correlation seems to work nicely also for f-block elements, some uncertainty still remains as for the parameters of the sp-metal oxides correlation owing to the limited numbers of oxides band gap values used to derive it as well as to the difficulty to get reliable optical band gap values for sp-metals having very low electronegativity parameters.

Table 3

Experimental Band Gap Values and Electronegativity Parameter Estimated by Using the d-Metal Correlation for a Group of Lanthanides Metals. ${ }^{40}$

\begin{tabular}{lcr}
\hline Phase & $\mathrm{E}_{\mathrm{g}} / \mathrm{eV}$ & $\chi_{\mathrm{M}}$ \\
\hline $\mathrm{Sm}_{2} \mathrm{O}_{3}$ & 5.0 & 1.31 \\
$\mathrm{Dy}_{2} \mathrm{O}_{3}$ & 5.0 & 1.31 \\
$\mathrm{Yb}_{2} \mathrm{O}_{3}$ & 5.2 & 1.27 \\
$\mathrm{Gd}_{2} \mathrm{O}_{3}$ & 5.3 & 1.26 \\
$\mathrm{La}_{2} \mathrm{O}_{3}$ & 5.4 & 1.24 \\
$\mathrm{CeO}_{2}$ & 5.5 & 1.22 \\
\hline
\end{tabular}




\section{(ii) Ternary Crystalline Oxides}

The most interesting aspect embodied in the proposed correlation is the possibility to use such relationships for predicting the band gap of mixed oxides so opening a new route to the quantitative characterization of corrosion layers on metallic alloys. It was suggested that Eq. (60) could be extended to ternary oxides, $\mathrm{A}_{\mathrm{a}} \mathrm{B}_{\mathrm{b}} \mathrm{O}_{\mathrm{o}}$, ${ }^{137}$ containing only $\mathrm{d}$,d-metal or sp,sp-metal oxides, by substituting to the metal electronegativity, $\chi_{M}$, the arithmetic mean for the cationic group, $\chi_{c}$, defined as:

$$
\chi_{\mathrm{c}}=\frac{a \chi_{\mathrm{A}}+b \chi_{\mathrm{B}}}{a+b}
$$

where $a$ and $b$ are the stoichiometric coefficients of the cations in the ternary oxide, and $\chi_{\mathrm{A}}$ and $\chi_{\mathrm{B}}$ their electronegativities.

In Table 4 the experimental band gap values for a number of binary and ternary d,d-metal oxides covering a quite large range of

Table 4

Experimental Optical Band Gaps Values for d-Metal and d-dMetals Alloys Oxides and Comparison Between Metal Electronegativity Estimated According to Eqs. (60a) and (61), $\chi_{\text {exp, }}$ and Pauling Electronegativity. ${ }^{40}$

\begin{tabular}{llll}
\hline Phase & $E_{g}^{\text {exp }}$ & $\chi_{\text {exp }}$ & $\chi_{\text {Pauling }}$ \\
& $(\mathrm{eV})$ & & \\
\hline $\mathrm{Y}_{2} \mathrm{O}_{3}$ & 5.50 & 1.22 & 1.20 \\
$\mathrm{Sc}_{2} \mathrm{O}_{3}$ & 5.40 & 1.24 & 1.30 \\
$\mathrm{CuYO}_{2}$ & 3.50 & 1.55 & 1.55 \\
$\mathrm{MnTiO}_{3}$ & 3.10 & 1.65 & 1.60 \\
$\mathrm{La}_{2} \mathrm{Ti}_{2} \mathrm{O}_{7}$ & 4.00 & 1.48 & 1.445 \\
$\mathrm{FeTiO}_{3}$ & 2.85 & 1.71 & 1.725 \\
$\mathrm{Y}_{3} \mathrm{Fe}_{5} \mathrm{O}_{12}$ & 3.00 & 1.68 & 1.64 \\
$\mathrm{CuScO}_{2} \mathrm{ScO}_{18}$ & 1.62 & 1.60 \\
$\mathrm{Fe}_{18} \mathrm{Ti}_{2} \mathrm{O}_{31}$ & 3.30 & 1.87 & 1.88 \\
$\mathrm{Fe}_{8} \mathrm{Ti}_{2} \mathrm{O}_{16}$ & 2.09 & 1.85 & 1.85 \\
$\mathrm{Fe}_{6} \mathrm{Ti}_{4} \mathrm{O}_{17}$ & 2.17 & 1.81 & 1.80 \\
$\mathrm{Fe}_{10} \mathrm{Ti}_{10} \mathrm{O}_{35}$ & 2.35 & 1.78 & 1.775 \\
$\mathrm{Fe}_{18} \mathrm{Ti}_{22} \mathrm{O}_{71}$ & 2.50 & 1.76 & 1.76 \\
\hline
\end{tabular}




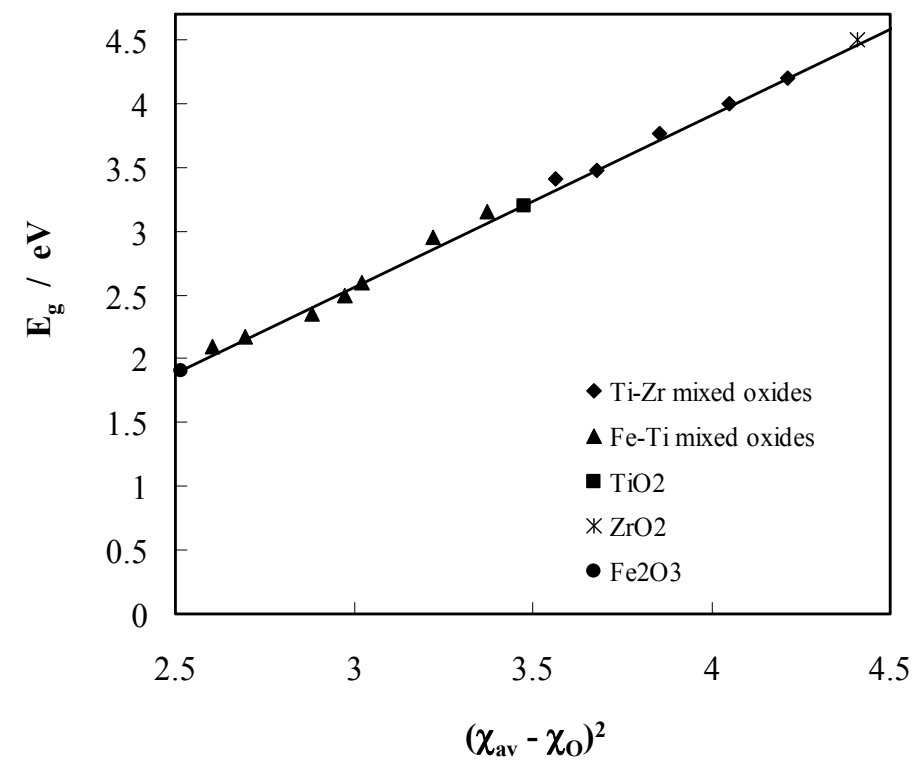

Figure 22. Experimental band gap values relative to Fe-Ti mixed oxides ${ }^{145}$ and Ti-Zr mixed oxides ${ }^{122}$ as a function of $\left(\chi_{\mathrm{av}}-\chi_{\mathrm{O}}\right)^{2}$. The continuous line represents the theoretical prediction according to Eq. (60)a.

band gap values $(1.90-5.50 \mathrm{eV})$ and d-metal electronegativity $(\Delta \chi$ $\cong 0.7)$ are reported. A very good agreement is observed between the $\chi$ experimental value obtained by PCS and the Pauling's ones. The difference between the two values stays always within the experimental uncertainty given in the Pauling's book. ${ }^{139}$ A further confirmation of the validity of the proposed correlation for d,dmetal ternary oxide comes from the data of Fig. 22 showing the fitting of the experimental $E_{g}$ values of ternary Ti-Fe and Ti-Zr mixed oxides as a function of different cationic ratio into the films. It is interesting to stress that for amorphous or disordered mixed oxides slightly larger $E_{g}$ values are experimentally observed (see also Section). By taking into consideration the results at our disposal for d-metal and d,d-metal mixed oxides we can reasonably state that Eq. (60a) together with Eq. (61) are able to predict the 
band gap of transition metal oxides within the experimental uncertainty.

Unfortunately we are not aware of a similar large range of band gap values for sp-metal mixed oxides. The few available data seem to confirm the validity of sp-metal correlation also for ternary oxides with differences in the experimental and calculated $E_{g}$ values in the order of $10 \%$.

As for ternary sp,d-metal oxides it was found that the d-metal correlation was able to fit quantitatively their band gap values provided that the difference in the electronegativity between the metallic cations is less than $0.5^{137}$ (see Table 5). Due to the limited number of systems investigated it remains unsolved the limits of applicability of such correlation to the sp,d-metal mixed oxides. Very recent results both for crystalline bulk $\mathrm{Mg}-\mathrm{Zn}^{142}$ oxides and amorphous anodic oxide on Al-W alloys ${ }^{143}$ alloys seem to suggest that when the atomic fraction of d-metal in the ternary oxides reaches values lower than $20 \%$ the experimental $E_{g}$ data are better interpolated by the sp-metal oxide correlation. These aspects need further investigations aimed to better define the limits of validity of the proposed semiempirical correlation.

Table 5

Experimental Optical Band Gaps Values for sp-d-Metal Alloys Oxides and Comparison Between Metal Electronegativity Estimated According to Eqs. (60) and (61), $\chi_{\text {exp }}$, and Pauling Electronegativity. ${ }^{40}$

\begin{tabular}{llll}
\hline Phase & $\begin{array}{c}E_{g}^{\text {exp }} \\
(\mathrm{eV})\end{array}$ & $\chi_{\text {exp }}$ & $\chi_{\text {Pauling }}$ \\
& 3.0 & & \\
\hline $\mathrm{Bi}_{0.7} \mathrm{Y}_{0.3} \mathrm{O}_{1.5}$ & 5.40 & 1.68 & 1.69 \\
$\mathrm{SrZrO}_{3}$ & 3.70 & 1.24 & 1.20 \\
$\mathrm{MgTiO}_{3}$ & 4.0 & 1.54 & 1.425 \\
$\mathrm{La}_{2} \mathrm{NiO}_{4}$ & 3.76 & 1.48 & 1.43 \\
$\mathrm{Mg}_{0.19} \mathrm{Zn}_{0.81} \mathrm{O}$ & 3.92 & 1.53 & 1.52 \\
$\mathrm{Mg}_{0.27} \mathrm{Zn}_{0.73} \mathrm{O}$ & 4.19 & 1.50 & 1.49 \\
$\mathrm{Mg}_{0.36} \mathrm{Zn}_{0.67} \mathrm{O}$ & & 1.45 & 1.46 \\
\hline
\end{tabular}




\section{(iii) Amorphous Oxide Films}

The previous correlation was extended to amorphous anodic films and corrosion layers by taking into account the influence of the amorphous nature on the optical band gap of passive films as previously discussed. Numerous investigations on anodic oxide films of valve metals have shown that amorphous oxides usually displayed optical band gap values larger than crystalline counterpart in absence of other specific defects. According to these results it has been proposed to take into account the influence of amorphous nature of passive films on their optical band gap values by modifying the correlation, used for crystalline oxides, as follows: ${ }^{144}$

$$
E_{g f}-\Delta E_{a m}=A\left(\chi_{c}-\chi_{O}\right)^{2}+B
$$

where $E_{g f}$ is the optical band gap of a passive film and $\Delta E_{a m}$ represents the difference between $E_{g f}$ and the optical band gap of the crystalline counterpart in $\mathrm{eV}$. The choice of $A, B$ and $\Delta E_{a m}$ depends on the nature (sp or d) of the metal cations as well as on their relative atomic fraction in the case of mixed oxides.

As for the influence of the lattice disorder on $E_{g}$ a value of $\Delta E_{a m} \leq 0.35 \sim 0.40 \mathrm{eV}$ seems able to account for the experimental data (see Table 1). $\Delta E_{a m}$ values in the order of $0.35 \sim 0.40 \mathrm{eV}$ are typical of truly amorphous oxides $\left(\mathrm{MoO}_{3}, \mathrm{WO}_{3}, \mathrm{Al}_{2} \mathrm{O}_{3}\right.$,), whilst lower values are expected for anodic film oxides having a tendency to grow in microcrystalline forms $\left(\mathrm{ZrO}_{2}, \mathrm{TiO}_{2}, \mathrm{Ta}_{2} \mathrm{O}_{5}\right)$. In Fig. 22 it is reported a fitting, according to Eq. (62), of the band gap values as a function of the compositional parameter $\chi_{\mathrm{c}}$ obtained according to Eq. (61) for two different ternary oxides systems containing only d-metal cations and forming both amorphous and crystalline phases as a function of cationic ratio into the film. The oxide films were obtained by anodizing metallic alloys (Ti- $\mathrm{Zr}$ magnetron sputtered alloys) or by metal-organic chemical vapour deposition. ${ }^{122,145}$ As previously mentioned in both cases a $\Delta \mathrm{E}_{\mathrm{am}}$ value near to zero, in agreement with the theoretical expectation, is derived for crystalline phases, whilst from the fitting of experimental data a $\Delta E_{a m}$ value different from zero is obtained for amorphous phases. 


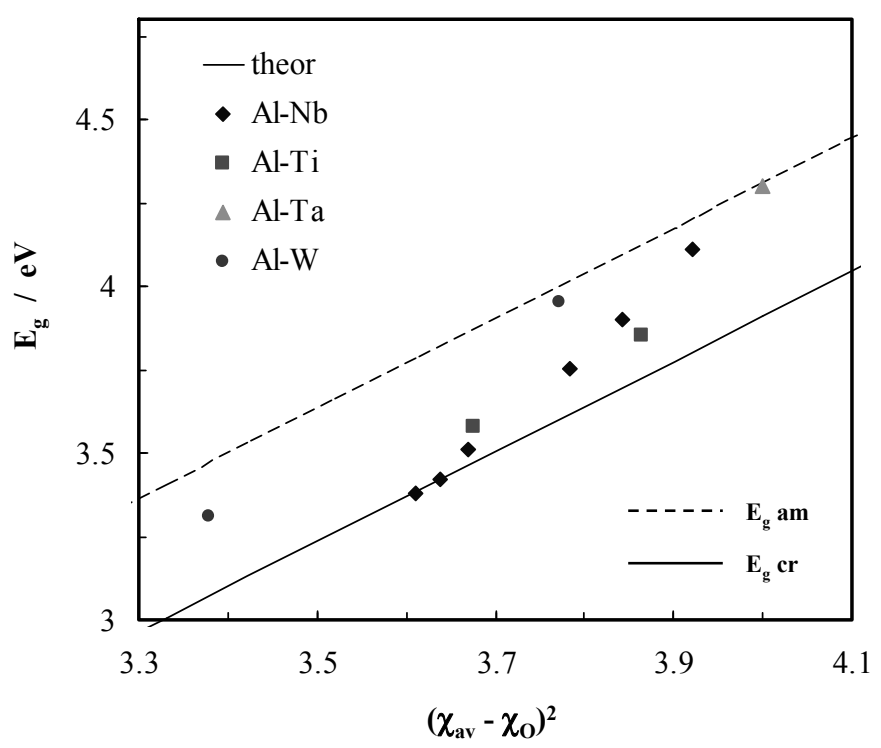

Figure 23. Experimental band gap values relative to Al-d metal mixed oxides $^{143}$ as a function of $\left(\chi_{\mathrm{av}}-\chi_{\mathrm{O}}\right)^{2}$. The continuous line represents the theoretical prediction according to Eq. (60)a. The dotted line represents the theoretical prediction for amorphous oxides with $\Delta E_{a m}=0.4 \mathrm{eV}$ to Eq. (62).

The value of $\Delta E_{a m}$, as previously mentioned, seems to be a measure of the degree of long range disorder provided that the oxide stoichiometry remains almost the same in the two phases. According to this systems showing a tendency to form microcrystalline phase display band gap values very near to the crystalline counterpart. This is evidenced in Fig. 23 where it is reported the fitting of the band gap values as a function of the compositional parameter $\chi_{c}$ obtained for different amorphous ternary oxides, containing both sp-metal (i. e. $\mathrm{Al}^{3+}$ ) and d-metal cations, grown by anodizing sputter-deposited or physical vapour deposited metal alloys. ${ }^{143}$ In both cases a $\Delta E_{a m}$ value in agreement with the theoretical expectation was derived from the fitting of experimental data and, significantly, larger $\Delta E_{a m}$ value were measured for anodic films made by oxides having a tendency to grow in truly amorphous state. Further evidence in favour of the proposed corre- 
lation for anodic films grown on Mo-Ta, Ti-Zr, Hf-W metallic alloys can be found in Refs. [144,146-148]. We have to mention that apart the nature of the oxide other experimental parameters can affect the lattice disorder degree of passive films and then the $\Delta E_{a m}$ value in anodic films. ${ }^{127,143,146}$

\section{(iv) Correlation for Hydroxides and Oxi-hydroxide Films}

For a further improvement toward the possibility to use PCS in a quantitative way for the investigation of corrosion layers grown in different conditions, we need to correlate the optical band gap of hydroxides and oxy-hydroxides to their composition. The experimental data collected on a number of systems suggest that hydroxides films display lower optical band gap values with respect to the corresponding anhydrous oxides. This finding can be rationalized on the basis of the correlation between the band gap of the films and the electronegativity of their constituents. In the case of hydroxide phases we can postulate that the band gap depends on the difference between the electronegativity of metal cation, $\chi_{\mathrm{M}}$, and hydroxyl group, $\chi_{\mathrm{OH}}$. The latter can be calculated as the arithmetic mean of those pertaining to oxygen (3.5) and hydrogen (2.2): accordingly a value of 2.85 is obtained.

In order to interpret some experimental data pertaining to passive layers on metals, for which the formation of hydroxides was inferred on the basis of PCS study and supported by thermodynamic considerations or confirmed by surface analytical investigations (XPS and XANES), we assumed ${ }^{137}$ that, in agreement with the findings on pure and mixed oxides, also for hydroxides the optical band gap value depends on the square of the difference between the electronegativity of the metallic cation and the average anionic electronegativity $\left(\chi_{\mathrm{M}}-\chi_{\mathrm{OH}}\right)$. In the electrochemical literature there were few, but experimentally well defined, investigated systems which seemed to support our assumption. Further studies, carried out in our laboratory on selected systems, have now provided a number of experimental data sufficient to derive numerical correlation regarding the optical band gap of hydroxide films on both sp- and d-metals. ${ }^{135,149,150}$ Figure 24 shows the photocurrent spectrum relating to an $\mathrm{Mg}(\mathrm{OH})_{2}$ film on mechanically polished magnesium formed by anodizing the metal in $0.1 \mathrm{M}$ 


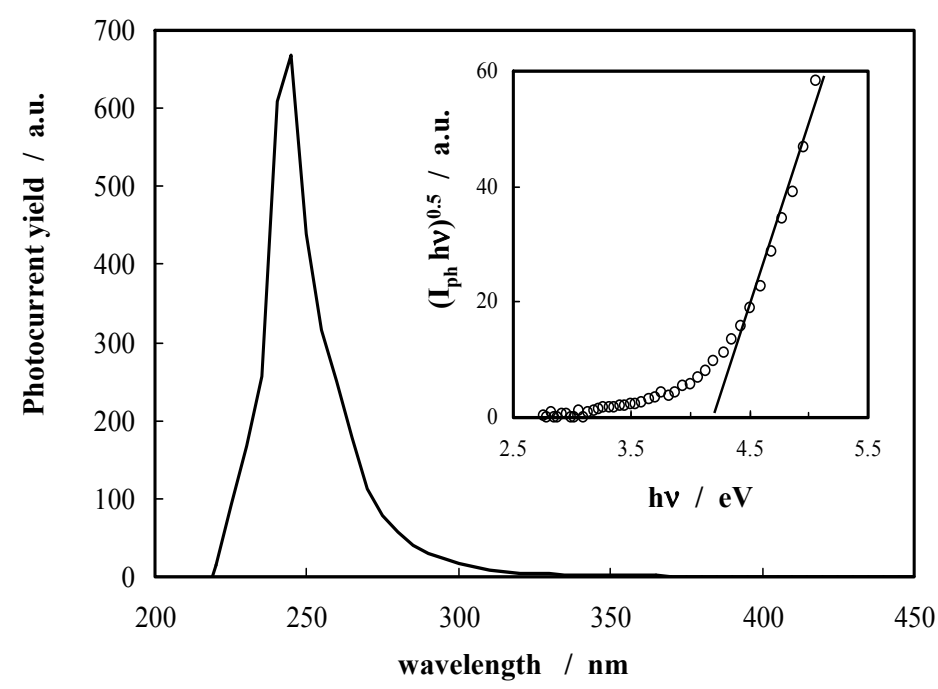

Figure 24. Photocurrent spectrum relating to $\mathrm{Mg}$ after mechanical treatment and anodizing in $0.1 \mathrm{M} \mathrm{NaOH}$ to $0.6 \mathrm{~V}(\mathrm{MSE})$, recorded by polarizing the electrode at the formation potential in the same electrolyte. Inset: $\left(I_{p h} h v\right)^{0.5}$ vs. $h v$ plot. Reprinted from M. Santamaria, F. Di Quarto, S. Zanna, and P. Marcus, "Initial surface film on magnesium metal: A characterization by X-ray photoelectron spectroscopy (XPS) and photocurrent spectroscopy (PCS)." Electrochim. Acta 53 (2008) 1314, Copyright (2007) with permission from Elsevier.

$\mathrm{NaOH}$ to $0.6 \mathrm{~V}(\mathrm{MSE})$ : an optical band gap of $4.25 \mathrm{eV}$ is determined, by assuming indirect optical transitions (see inset of the figure), a value well below the value of $7.8 \mathrm{eV}$, now widely accepted, for $\mathrm{MgO}$. On the basis these experimental data, ${ }^{149}$ the following correlation has been proposed:

$$
\mathrm{E}_{\mathrm{g}}=1.21\left(\chi_{\mathrm{M}-} \chi_{\mathrm{OH}}\right)^{2}+0.90
$$

for hydroxides grown on sp-metal, and:

$$
\mathrm{E}_{\mathrm{g}}=0.65\left(\chi_{\mathrm{M}-} \chi_{\mathrm{OH}}\right)^{2}+1.38
$$

for hydroxides grown on d-metals. It is noteworthy that, as previously reported for metal oxides, different relationships hold for spmetal and d-metal hydroxides. With respect to the analogous cor- 
relation obtained for sp-metal oxides (Eq. 60), the most relevant difference in Eq. (63a) comes out from the second term of the right side, suggesting that sp-metal hydroxides present always a finite optical band gap, in the order of $1.70 \mathrm{eV}$ for $\mathrm{Tl}$, the most electronegative sp-metals $(\chi=2.03)$. Although this last correlation suffers some limitations owing to the rather limited number of investigated systems it is able to rationalise some results reported in the literature. In fact from Eq. (63a) an optical band gap around 2.25 $\mathrm{eV}$ is derived for $\mathrm{Ni}(\mathrm{OH})_{2}$ by assuming $\chi_{\mathrm{Ni}}=1.80$ according to the Pauling scale and under the hypothesis that also for Ni-hydroxides holds the sp-metal correlation valid for $\mathrm{NiO}$. The optical gap estimated according to Eq. (63a) is in good agreement with the value estimated from photocurrent spectra reported in the literature ${ }^{151}$ for passive films on $\mathrm{Ni}\left(E_{g}=2.20 \mathrm{eV}\right)$ and for $\mathrm{Ni}(\mathrm{OH})_{2}$ electrochemically deposited and soaked in $\mathrm{KOH}$ solution. ${ }^{152}$ These findings confirm once more that in the proposed correlation $\mathrm{Ni}$ (II) oxide and hydroxide conforms to the sp-metal behavior.

Further support to the proposed hydroxides correlations comes from more recent data obtained from the PCS study of conversion coating grown on $\mathrm{Mg}$ metal in stannate bath, where the formation of a mixed $\mathrm{MgSn}(\mathrm{OH})_{6}$ layer has been reported, ${ }^{153}$ as well as from PCS characterisation of $\mathrm{Co}(\mathrm{OH})_{2}$ and $\mathrm{La}(\mathrm{OH})_{3}$ films grown in alkaline solution. ${ }^{150,154}$ Owing to the lack of information on the band gap values of a larger number of hydroxides, the correlation between the optical band gap and the difference of electronegativity of the hydroxides constituents are based on a restrict number of systems and, thus, it must be taken with some caution. Nevertheless, it is possible to make some general considerations on the behavior of some investigated systems lending a further support to their validity.

By comparing the correlation relative to transition metals, it comes out that larger band gap values are expected for the hydroxide phase than for the corresponding anhydrous oxide, when the cation electronegativity is higher than 1.95 . Such a finding can help to rationalize the experimental results reported for gold electrode as well as for other noble metals. In the case of gold an optical band gap of about $1.50 \mathrm{eV}$ is expected for the hydroxide phase in comparison with the near to zero band gap value foreseen for the anhydrous oxide. Analogously, for platinum metal $\left(\chi_{M}=2.1\right)$, an increase of the optical gap is calculated from our correlations on 
going from the oxide $\left(E_{g}=1.16 \mathrm{eV}\right)$ to the hydroxide phase $(1.75$ $\mathrm{eV})$. Although the nature and the photoelectrochemical behavior of the oxide films grown on these noble metals are still under debate ${ }^{39}$ this finding could help to explain the complex behavior observed experimentally.

The last point we like to discuss is the possibility to relate the band gap values of passive films to their hydroxylation degree. At this aim it has been suggested ${ }^{137}$ on a purely heuristic basis, a connection formula between the band gap of oxides and the band gap of the corresponding oxi-hydroxide, $\mathrm{MO}_{(\mathrm{y}-\mathrm{m})} \mathrm{OH}_{(2 \mathrm{~m})}$. According to this suggestion it has been proposed that the band gap of anhydrous and hydroxilated oxide phase can be related trough the relationship:

$$
E_{g}^{h y d}=\frac{E_{g}^{a n h}}{1+k_{m} x_{\mathrm{OH}}}
$$

where $k_{m}$ is a constant that can be calculated for each system once the band gap values of the anhydrous oxide and hydroxide are known. $x_{\mathrm{OH}}$ is the fraction of hydroxyl group into the oxihydroxide phase defined as:

$$
x_{\mathrm{OH}}=\frac{2 m}{y+m}
$$

It is interesting to note that Eqs. (64) and (65) have been successfully used to get the composition of Chromate Conversion Coating (CCC) formed on aluminium from their band gap values as a function of the hydration degree and as a function of the $\mathrm{Cr}(\mathrm{III}) / \mathrm{Cr}(\mathrm{VI})$ ratio. $^{155}$ The photoelectrochemical characterization of CCC grown for different conversion times and in different electrochemical baths data showed that a quite reproducible optical band gap value, around $2.55 \mathrm{eV}$, is derived for freshly prepared CCCs. Such a value is different from that measured for passive chromium $^{155}$ and assigned to $\mathrm{Cr}(\mathrm{OH})_{3}$ anodic film $(2.40 \mathrm{eV})$, and has been attributed to the formation of an amorphous $\mathrm{Cr}(\mathrm{III})$ $\mathrm{Cr}(\mathrm{VI})$ mixed oxy-hydroxide of formula $\mathrm{Cr}_{0.667} \mathrm{Cr}_{0.333} \mathrm{O}_{1.2}(\mathrm{OH})_{1.6}$, 
having a stoichiometric ratio $\mathrm{O} / \mathrm{Cr}$ equal to 2.80 , in fair agreement with data obtained by XANES and Auger spectroscopy. ${ }^{155}$

\section{(v) PCS Analysis of Passive Films and Corrosion Layers on Base Metals and Alloys}

One of the most challenging task for any experimental analytical technique is to be able to provide useful information on the electronic properties and chemical composition of passive films on base metals (like: $\mathrm{Cu}, \mathrm{Fe}, \mathrm{Cr}, \mathrm{Ni}$ ) and their alloys (stainless steels (SS), Fe-Cr alloys, etc.). The previous correlation has been used to relate quantitatively PCS data to passive films composition formed on some of these metals. ${ }^{131,137,156-159}$ In the last years an increasing use of such correlation has been registered in PCS studies aimed to characterize passive film on base metals and alloys (Fe-Cr, SS). ${ }^{160-}$ ${ }^{166}$ In these complex systems further information on the oxidation state of each metallic cation and their atomic fraction can be necessary in order to use quantitatively the previous correlation. Moreover in some cases the expected optical band gap values of oxides and hydroxides of the same metal, at different oxidation states, are very close or almost coincident so that it becomes difficult to get quantitative information from PCS measurements alone.

In order to highlight this point, in Table $6 \mathrm{a}$ we report the band gap values of crystalline oxides and hydroxides of a group of base metals $(\mathrm{Cu}, \mathrm{Cr}, \mathrm{Fe}, \mathrm{Ni})$ experimentally measured by PCS technique or derived from the literature. In the same table we report the Pauling electronegativity parameter for the different oxidation states. Apart from the electronegativity value of $\mathrm{Cr}(\mathrm{VI})$ for which we are not aware of other reliable values in literature, we like to stress that the electronegativity data obtained by Eq. (60a) are in very good agreement with those reported in literature ${ }^{139-141}$ so that we suggest to use these last values as preferred PCS electronegativity parameters for characterisation of passive films on pure base metals and on their alloys. According to this we reported in Table $6 \mathrm{~b}$ the estimated band gap values of the corresponding hydroxides as derived from the proposed correlation and by using the PCS preferred $\chi_{\mathrm{M}}$ values.

It comes out from data of Table 6 that oxides and hydroxides of d-metal having electronegativity around 1.90 display very close band gap values, so making very difficult to distinguish by PCS 
Table 6

(a)Band Gap Values of Crystalline Oxides and Hydroxides of a Group of Base Metals. Comparison Between Pauling Electronegativity and PSC Estimated Electronegativity. ${ }^{40}$

\begin{tabular}{llcc}
\hline Phase & $\begin{array}{c}E_{g} \\
(\mathrm{eV})\end{array}$ & $\chi_{\text {Pauling }}$ & $\chi_{\text {PCS }}$ \\
\hline $\mathrm{Cr}_{2} \mathrm{O}_{3}$ & 3.30 & 1.60 & 1.62 \\
$\mathrm{Cr}(\mathrm{OH})_{3}$ & 2.43 & 1.60 & 1.62 \\
$\mathrm{CrO}_{3}$ & 2.0 & - & 1.89 \\
$\mathrm{ZnO}$ & 3.34 & 1.60 & 1.60 \\
$\mathrm{Cu}$ & 1.86 & 1.90 & 1.90 \\
$\mathrm{CuO}$ & 1.40 & 2.00 & 2.04 \\
$\mathrm{FeO}$ & 2.40 & 1.80 & 1.80 \\
$\mathrm{Fe} \mathrm{O}_{3}$ & 1.90 & 1.90 & 1.91 \\
$\mathrm{NiO}$ & 3.80 (direct) & 1.80 & 1.80 \\
& 3.58 (indirect) & & 1.77 \\
$\mathrm{Ni}(\mathrm{OH})_{2}$ & 2.31 & 1.80 & 1.77 \\
\hline
\end{tabular}

(b) Band gap values of some base metal hydroxides estimated according to Eq. (63)b.

\begin{tabular}{lcc}
\hline Phase & $\chi_{\text {PCS }}$ & $\begin{array}{r}E_{g} \\
(\mathrm{eV})\end{array}$ \\
\hline $\mathrm{Zn}(\mathrm{OH})_{2}$ & 1.60 & 2.39 \\
$\mathrm{CuOH}$ & 1.90 & 1.97 \\
$\mathrm{Cu}(\mathrm{OH})_{2}$ & 2.04 & 1.80 \\
$\mathrm{Fe}(\mathrm{OH})_{2}$ & 1.80 & 2.10 \\
$\mathrm{Fe}(\mathrm{OH})_{3}$ & 1.91 & 1.95 \\
\hline
\end{tabular}

alone which type of passive films is formed. This is the case of copper and iron passive films for which at least three different phases at different hydroxylation degree exist with very close optical band gap values. In such a case further information gathered by other (possibly in situ) analytical techniques are necessary to rationalise PCS data of passive films.

Other authors ${ }^{167-169}$ have proposed a different route for the identification of passive film. According to these authors a comparison of PCS data (as well as of the general semiconducting behavior) between passive films anodically grown and sputtered oxides could help to identify the nature of passive films. This approach may be useful provided that any difference in the defect 
structure and hydroxylation degree for oxide phases grown in such different ways is negligible.

The data of Table 6 and the previous considerations could help to rationalise some of the experimental findings on PCS analysis concerning passive films on iron, for which band gap values ranging between $1.90 \mathrm{eV}$ and $2.10 \mathrm{eV}$ are reported in the literature. ${ }^{169} \mathrm{~A}$ band gap value of $1.90 \mathrm{eV}$ has been estimated for passive films formed on iron in borate buffer solution $(\mathrm{pH}=8.4)$ at high potential $\left(U_{E}=0.6 \sim 0.8 \mathrm{~V}(\mathrm{SCE})\right) .{ }^{169}$ This value matches well the one reported in the same work for evaporated $\mathrm{Fe}_{2} \mathrm{O}_{3}$ and the one reported in Table 6a for crystalline $\mathrm{Fe}_{2} \mathrm{O}_{3}$, thus allowing identifying the passive film composition as $\mathrm{Fe}(\mathrm{III})$ oxide. This conclusion is in agreement with recent XANES data suggesting the formation of micro-crystalline iron oxide structure (LAMM phase) ${ }^{170}$ on passive iron anodized under similar conditions. The higher $E_{g}$ measured at lower polarizing voltage $\left(U_{E}<0.4 \mathrm{~V}(\mathrm{SCE})\right)$ have been be ascribed to the presence of crystallographic disorder, as in amorphous passive films, or to the partial reduction of $\mathrm{Fe}$ (III) to $\mathrm{Fe}(\mathrm{II})$ with a subsequent variation of film composition. ${ }^{171}$ According to Eqs. (60a) and (61) a band gap of $2.0 \mathrm{eV}$ is expected for a $10 \% \mathrm{Fe}$ (II) containing oxide in agreement with the value reported in Ref. [169] for oxidised magnetite, while $E_{g}=2.1 \mathrm{eV}$ can be estimated for $\mathrm{Fe}_{3} \mathrm{O}_{4}$ in contrast with the value reported in the same work. The $E_{g}$ variation with $U_{E}$, reported in Ref. [169], is predicted by the above mentioned equations as a function of the Fe(II) content into the film, and it should agree with the description of passive film on iron, described as a $\mathrm{Fe}(\mathrm{II})$ deficient magnetite of formula $\mathrm{Fe}(\mathrm{II})_{1-\mathrm{x}} \mathrm{Fe}(\mathrm{III})_{2} \mathrm{O}_{4-\mathrm{x}}$, with $\mathrm{Fe}(\mathrm{II})$ content in the range of 10$25 \%$ depending on the passivation potential. ${ }^{169}$

By considering that a band gap value around of $1.90 \pm 0,05$ $\mathrm{eV}$ has been estimated for $\mathrm{Fe}(\mathrm{III})$ oxide and hydroxide, almost coincident with that of $\mathrm{Fe}_{2} \mathrm{O}_{3}$ and $\mathrm{Fe}(\mathrm{OH})_{3}$ is estimated in Table 6, we can conclude that $E_{g}=1.85 \sim 1.95 \mathrm{eV}$ is expected for iron passive films having composition equal to $\mathrm{FeOOH}$, and thus PCS is not sensitive to variation of the hydration degree of $\mathrm{Fe}$ passive films due to the small dependence of the band gap values on the $\mathrm{OH}$ content. A detailed discussion of the influence of anodizing solution, spanning a large range of $\mathrm{pH}(1 \leq \mathrm{pH} \leq 15)$, and electrode potential values on the measured optical band gap of passive film on metallic iron can be found in Ref. [171]. 
The use of PCS in the identification of passive films on ironchromium-nickel alloys and stainless steel appears even more difficult, despite the appreciable differences in the band gap values of the corresponding pure crystalline oxides. The possible formation of a passive film with an unknown hydration degree and with different metals at different oxidation state makes the PCS analysis very complex. By the way, different photoelectrochemical data on such systems are reported in the literature and the interested reader can access to the published works. ${ }^{104,160-166,172-183}$

Owing to space limitations we will discuss shortly and qualitatively some selected PCS data pertaining to such systems, which can be compared in some extent to more recent quantitative analysis based on in situ and ex situ techniques (XANES, XPS, EQCM). ${ }^{184-187}$

The formation of $\mathrm{Cr}(\mathrm{OH})_{3}$ on passive $\mathrm{Cr}$ at low potentials $\left(U_{E}\right.$ $\leq-0,6 \mathrm{~V}(\mathrm{MSE}))$ and in a wide range of $\mathrm{pH}(0<\mathrm{pH}<9)$ has been suggested on the basis of the measured optical band gap value $\left(E_{g}\right.$ $=2.45 \pm 0.1 \mathrm{eV}$, see Refs. [159.160-162,188]). Such a hypothesis is in agreement with XPS and XANES data reported in Refs. [184-187] for films grown on $\mathrm{Cr}$ and on $\mathrm{Fe}-\mathrm{Cr}$ alloys in acidic solutions as well as in slightly alkaline solutions. ${ }^{189}$

As for PCS data of passive films grown on $\mathrm{Fe}-\mathrm{Cr}$ alloys $(\mathrm{Cr}$ content $\leq 30$ at. $\%)$ in borax buffer solution $(8.4 \leq \mathrm{pH} \leq 9.2)$ band gap values ranging from $1.90 \mathrm{eV}$ to $2.20 \mathrm{eV}$ with increasing $\mathrm{Cr}$ content have been reported in Refs. [177,181]. In a more recent work, ${ }^{190}$ by using steady-state PCS data band gap around $3.0 \mathrm{eV}$ have been reported for passive film on $\mathrm{Fe}-\mathrm{Cr}$ alloys $(\mathrm{Cr}$ content $\leq$ 20at.\%) at variance with the previous ones. Such difference in $E_{g}$ values could be attributable, in principle, to a loss of sensitivity in the last method, which could miss to detect the lower photocurrent values usually measured for photon energies near the band gap of passive films. This last hypothesis could also explain higher $E_{g}$ values measured, for passive films grown on experimental ferritic $\mathrm{SS}^{174}$ in chloride containing solution, at low anodic potentials $\left(E_{g}\right.$ $=2.80 \mathrm{eV})$. On the other hand $E_{g}$ values around $2.60 \mathrm{eV}$ were measured, at higher electrode potentials $\left(U_{E}=0.6 \mathrm{~V}(\mathrm{SCE})\right)$, for passive films formed on analogous ferritic SS containing small amount $(<4 \%$ in weight $)$ of Mo and $\mathrm{Ni}^{172}$

Due to the large range of $E_{g}$ values collected in the literature for passive films on Fe-Cr alloys, the loss of sensitivity of PCS for 
photon energies near the band gap of passive films does not seems a satisfactory explanation. The data on passive films composition obtained by in situ and ex situ analytical techniques reported in Refs. [184-187] for Fe-Cr passive films grown at $\mathrm{pH}$ values $\leq 4.5$ can be useful to justify these results.

XANES data on various $\mathrm{Fe}-\mathrm{Cr}$ alloys ${ }^{184}$ strongly suggest that at $\mathrm{pH}=4.5$ (acetate buffer solution) films formed at low potentials $\left(U_{e}=-0.3 \mathrm{~V}(\mathrm{MSE})\right)$ are enriched, with respect to the base alloy, of $\mathrm{Cr}$ (III) usually present in the films as hydrated species, whilst at higher potentials $\left(U_{E}=0.2 \mathrm{~V}(\mathrm{MSE})\right)$ the passive films display both a minor $\mathrm{Cr}$ enrichment as well as a decrease in hydroxides content. These findings suggest that passive films on $\mathrm{Fe}-\mathrm{Cr}$ alloys are oxides containing both the metals with a $\mathrm{Fe} / \mathrm{Cr}$ ratio and a hydration degree dependent on experimental conditions. In a more recent paper ${ }^{187}$ the formation of $\mathrm{Cr}(\mathrm{OH})_{3}$ in acidic solution of $0.5 \mathrm{M}$ $\mathrm{H}_{2} \mathrm{SO}_{4}$ has been reported on the basis of a XPS analysis of passive film on $\mathrm{Fe} 20 \mathrm{Cr}$ grown at various potentials. More interestingly in the same paper the formation of a chromite $\left(\mathrm{Fe}(\mathrm{II}) \mathrm{Cr}_{2} \mathrm{O}_{4}\right)$ compound at anodic potential near to $+0.4 \mathrm{~V}(\mathrm{SHE})$ was suggested. At still higher potential $\mathrm{U}_{\mathrm{E}}>0.8 \mathrm{~V}(\mathrm{SHE})$ the co-presence of $\mathrm{Fe}(\mathrm{III})$ and $\mathrm{Cr}(\mathrm{III})$ oxide species was suggested in presence of a decreasing amount of $\mathrm{Fe}(\mathrm{II})$ and $\mathrm{Cr}(\mathrm{OH})_{3}$ species. A band gap value near to $2.5 \mathrm{eV}$, very near to that estimated for $\mathrm{Cr}(\mathrm{OH})_{3}$, has been reported in Ref. [160] for a film grown in acidic solution on similar alloy, whilst both the presence of an optical band gap near to 2.5 $\mathrm{eV}$ as well as a second optical band gap around $3.0 \mathrm{eV}$ have been reported in Ref. [161] for Fe-18Cr passive film formed in borate buffer solution. We like to mention that as for chromite $\left(\mathrm{Fe}(\mathrm{II}) \mathrm{Cr}_{2} \mathrm{O}_{4}\right)$ compound an optical band gap around $3.0 \mathrm{eV}$ is foreseen according to the correlation previously reported, whilst for mixed $\mathrm{Fe}(\mathrm{III})-\mathrm{Cr}(\mathrm{III})$ oxides at the different composition near those reported in Ref. [187] band gap values ranging between 2.5 $\mathrm{eV}$ and $3.0 \mathrm{eV}$ can be estimated on the basis of the correlation for mixed d-metal oxides. It is also interesting that lower $E_{g}$ values pertain to the passive film grown on $\mathrm{Fe}-\mathrm{Cr}$ alloys at highest electrode potential. By extending the previous considerations to passive films on ferritic SS, the decrease of their optical band gap with increasing electrode potential can be analogously explained by an increase of the iron content in the passive film. 
According to Eqs. (60), (61) and (64), band gap around $2.1 \pm$ $0.1 \mathrm{eV}$ strongly point toward the presence on the surface of $\mathrm{Cr}-\mathrm{Fe}$ alloys of passive films constituted of mixed iron-chromium hydroxides richer in iron, with larger $E_{g}$ values attributable to passive film at higher $\mathrm{Cr}$ content.

As for passive films on austenitic SS $(\mathrm{Fe}-18 \mathrm{Cr}-8 \mathrm{Ni})$ and $\mathrm{Ni}-\mathrm{Cr}$ alloys (alloy 600 series), formed at high anodic potential $\left(U_{E}=0.8\right.$ $\mathrm{V}(\mathrm{SCE})$ ) in borate buffer solution at $\mathrm{pH}=9.2$, a rather constant optical band gap $\left(E_{g}=2.35 \pm 0.1 \mathrm{eV}\right)$ has been reported in Refs. [181-183], with the highest values for passive films on SS at higher Ni content or on Ni-Cr alloys. The $E_{g}$ value suggests that also in this case a chromium hydroxide rich phase is the main component of the passive films. At lower potentials $\left(U_{E}<0.1\right.$ $\mathrm{V}(\mathrm{SCE}))$ higher band gap values $\left(E_{g}=2.75 \pm 0.1 \mathrm{eV}\right)$ have been found. ${ }^{178,180}$ However, passive films on austenitic SS with higher molybdenum content ( $>4 \%$ in weight) usually displayed a more limited variation in the $E_{g}$ values as a function of electrode potentials. ${ }^{174,178}$ This finding was particularly evident in the case of the superaustenitic commercial alloy 254-SMO (20Cr-18Ni-6Mo), which displayed an almost constant $E_{g}$ value $(2.45 \pm 0.1 \mathrm{eV})$ in a rather large range of electrode potential $\left(-0.1 \leq U_{E} \leq 0.9 \mathrm{~V}(\mathrm{SCE})\right)$ at $\mathrm{pH}=6.5$ and in presence of chloride. ${ }^{174}$ The beneficial action of Mo in improving the pitting resistance of SS is well known ${ }^{191}$ and, according to PCS data, it could be ascribed to its ability to keep on the surface of such an alloy a passive film having an almost constant composition (very close to $\mathrm{Cr}(\mathrm{OH})_{3}$, according to the $E_{g}$ values) at different potentials. This interpretative hypothesis is in qualitative agreement with the results of STM and XPS analysis reported in Ref. [185] showing that the passive film grown on Fe$18 \mathrm{Cr}-13 \mathrm{Ni}$ in $0.5 \mathrm{M} \mathrm{H}_{2} \mathrm{SO}_{4}$ at $0.5 \mathrm{~V}$ (SHE) keeps a stable thicker outer layer containing almost chromium hydroxide also at longer polarization times. It seems reasonable to conclude that in the case of 254-SMO alloy the external layer of the passive film formed at $\mathrm{pH}=6.5$ and in presence of chloride ions in solution has a composition close to $\mathrm{Cr}(\mathrm{OH})_{3}$, as reported for the outer layer of the FeCr-Ni alloy discussed in Ref. [185].

Higher $E_{g}$ values (>2.40 eV) are expected for passive films on austenitic SS at higher potentials in presence of a dehydration process and/or for the formation of an anhydrous $\mathrm{Cr}(\mathrm{III})$ richer mixed oxides. ${ }^{185,192}$ However, very recently, in a series of papers 
dealing with the PCS characterization of passive film grown in borate solution on different SS (254 SMO, AISI 304L and AISI 316L) Blackwood and coworkers have suggested the possible presence of a surface layer of $\mathrm{Fe}_{2} \mathrm{O}_{3}$ at higher anodic potential and a duplex structure with a chromite-like inner phase underlying the external $\mathrm{Fe}_{2} \mathrm{O}_{3}$ layer at intermediate electrode potentials. At still more cathodic potential the possible formation of a p-type $\mathrm{FeO}$ oxide on the surface of the alloy 254SMO austenitic SS was suggested. These results indicate that the nature of passive films could be strongly dependent also from the nature of ionic species present in solution apart from the solution $\mathrm{pH}$. Further studies are necessary for reaching final conclusions on particular systems.

However, all the above mentioned results highlight both the ability of PCS technique to scrutinize very complex systems as well as the possibility to extract semi-quantitative information on the surface layer composition not conflicting with experimental data obtained by other very sophisticated techniques. We are confident that future PCS investigations on carefully chosen systems could provide further evidence in favour of a widespread use of such a technique in passivity and corrosion studies.

\section{CONCLUSIONS}

A critical analysis of the experimental results on the electronic properties of passive film, obtained by using a traditional approach based on the Mott-Schottky theory, has shown some aspects seriously conflicting with the theoretical hypothesis underlying the validity of the M-S analysis. In this review it has been shown that a better unifying framework for getting out information on electronic properties of passive films is provided by the theory of a-SC Schottky barrier. In the frame of this theory the differential admittance behavior of passive film/electrolyte junction can be qualitatively and quantitatively analyzed providing important information useful to locate the energy levels of the passive film/electrolyte junction as well as new insight on the DOS distribution inside the film.

As for the location of energy levels of the junction, in the case of amorphous or strongly disordered material, they can be seriously in error if the analysis of measured capacitance is carried out by 
means of traditional M-S approach. Moreover such an approach is not able to explain, neither qualitatively, the changes in the capacitance behavior observed at different $a c$ frequencies of electrical signal.

It has been suggested that in presence of very thin passive film, like those reported for base metal and alloys, a different approach, as that suggested by Gerischer for crystalline graphite electrodes, could offer a coherent approach to the study of the highly doped crystalline passive film formed on these material. It appears however rather surprising that, in the case of well documented systems having strongly disordered or amorphous nature, many researchers still employ the classical M-S analysis in spite of the fact that a well developed theory of amorphous semiconductor Schottky barrier has been developed more than 25 years ago, at least, for bulk amorphous semiconductors. Some further refinements to this last theory appear necessary for a quantitative analysis of the electronic properties of amorphous thin passive film in order to account for the limited thickness of the passive film and for the possible metal capacitance contribution to the measured capacitance of the film/electrolyte junction. Once such refinements will be carried out, it is our opinion that we could become able to get more insight also on the influence of anodizing process and post-anodizing treatment on the electronic properties of passive film grown on base-metal and valve-metal too. This last class of materials, which are of large interest in the electronic industry owing to their high $\mathrm{k}$ values, and specially anodic niobia and tantala deserve more investigation owing to the fact that they display some attractive features making them ideal candidates for testing further improvements in the theory of a-SC Schottky barriers.

In this review we presented also a relatively simple approach to the fundamentals of PCS technique currently used for characterizing passive films and corrosion layers on metal and alloys. Some of the interpretative models suggested in the past by the present authors to rationalize the experimental PCS data, gathered from many investigated systems, have been validate by different authors but some of them are still under scrutiny and they should be used with some caution. This is particularly true for the proposed correlation between optical band gap of hydroxides and difference of electronegativity of the constituents owing to the still limited, although increasing, number of investigated systems. 
Nevertheless, it is in our opinion that, PCS technique remains one of the most versatile techniques at our disposal, for getting out information on the solid-state properties of passive layers in corrosion studies.

The combined use of DA and PCS techniques in the characterization of thin and thick passive films on metal and alloys it has, already, provided important information, on electronic and solid state properties of films, which cannot be accessible by other techniques. If we consider that both techniques are able to test in situ real corroding systems at different time scales and electrode potential window, without any special procedure, it appears reasonable to predict a still wider use of both techniques in corrosion and electrochemical material science.

We are aware that, in many cases, other in situ and ex situ scanning probes having very high spatial resolution or vacuum techniques providing more precise information on the morphology or chemical composition of thin passive layers will be mandatory, but we have no doubts that DA and PCS techniques, together with other electrochemical techniques, will play an unchallenged role for a complete physico-chemical characterization of passive films and corrosion layers.

\section{REFERENCES}

${ }^{1}$ D. Landolt, Corrosion and Surface Chemistry of Metals, EPFL Press, Lausanne, CH, 2007.

${ }^{2}$ H.-H. Streblow, in Advances in Electrochemical and Engineering Science, R.C. Alkire, D.M. Kolb, Editors, Vol. 8, Wiley-VCH, Weinheim, FRG, 2003, p. 271

${ }^{3}$ Passivity of Metals, Proceedings of the $4^{\text {th }}$ International Symposium on Passivity, R.P. Frankental, J. Kruger, Editors, The Electrochemical Society Inc., Pennington, NJ, 1978.

${ }^{4}$ Passivity of Metals and Semiconductors, Proceedings of the $5^{\text {th }}$ International Symposium on Passivity, M. Froment, Editor, Elsevier, Oxford, 1983.

${ }^{5}$ Passivity of Metals and Semiconductors, Proceedings of the $6^{\text {th }}$ International Symposium on Passivity, N. Sato, K. Hashimoto, Editors, Pergamon Press, Oxford, 1990.

${ }^{6}$ Oxide Films on Metals and Alloys, B.R. MacDougall, R.S. Alwitt, T.A. Ramanarayanan, Editors, PV 92-22, The Electrochemical Society Inc., Pennington, NJ, 1992; Oxide Films on Metals and Alloys, K. Hebert, G.E. Thompson, Editors, PV 94-25, The Electrochem Society Inc., Pennington, NJ, 1994. 
${ }^{7}$ Passivity of Metals and Semiconductors, Proceedings of the $7^{\text {th }}$ International Symposium on Passivity, K.E. Heusler, Editor, Trans Tech. Publications Ltd., Zurich, 1995.

${ }^{8}$ H.H. Uhlig Memorial Symposium, F. Mansfeld, A. Asphahani, H. Böhni, R. Latanision, Editors, PV 94-26, The Electrochem Society Inc., Pennington, NJ, 1995.

${ }^{9}$ Passivity and its Breakdown, P. Natishan, H. S. Isaacs, M. Janik-Czackor, V. A. Macagno, P. Marcus, M. Seo, Editors, PV 97-26, The Electrochemical Society Inc., Pennington, NJ, 1998.

${ }^{10}$ Critical Factors in Localized Corrosion III, R. G. Kelly, G. S. Frankel, P. M. Natishan, R.C. Newman, Editors, PV 98-17, The Electrochemical Society Inc., Pennington, NJ, 1999.

${ }^{11}$ Passivity and Localized Corrosion, M. Seo, B. MacDougall, H. Takahashi, R.G. Kelly, Editors, PV-99-27, The Electrochemical Society Inc., Pennington, NJ, 1999.

${ }^{12}$ Passivity of Metals and Semiconductors, Proceedings of the $8^{\text {th }}$ International Symposium on Passivity, M. B. Ives, J.L. Luo, J.R. Rodda, Editors, PV-99-42, The Electrochemical Society Inc., Pennington, NJ, 2001.

${ }^{13}$ Surface Oxide Films, V. Birss, L.D. Burke, A.R. Hillmann, R.S. Lillard, Editors, PV-2003-25, The Electrochemical Society Inc., Pennington, NJ, 2004.

${ }^{14}$ Passivity of Metals and Semiconductors, and properties of Thin Oxide Layers, Proceedings of the $9^{\text {th }}$ International Symposium on Passivity, P. Marcus, V. Maurice, Editors, Elsevier, Amsterdam, 2006.

${ }^{15}$ W. H. Brattain, C.G.B. Garrett, Bell System Tech. J., 34 (1955) 129; Phys. Rev., 99 (1955) 177.

${ }^{16}$ M. Green, in Modern Aspects of Electrochemistry, Ed. by B.E. Conway, J.O'M. Bockris, Butterworths, London, 1959, vol. 2, p. 343.

${ }^{17} \mathrm{H}$. Gerischer, in Advances in Electrochemistry and Electrochemical Engineering, Ed. by P. Delahay, Interscience Publishers, New York, 1961, Vol.1, p. 139.

${ }^{18}$ V. A. Myamlin, Yu. V. Pleskov, Electrochemistry of Semiconductors, Plenum Press, New York, 1967.

${ }^{19}$ H. Gerischer, in Physical Chemistry. An Advanced Treatise, Ed. by H. Eyring, D. Henderson, W. Jost, Academic Press, New York, 1970, vol. IXA, p. 463.

${ }^{20}$ S.R. Morrison, Electrochemistry at Semiconductor and Oxidized Metal Electrodes, Plenum Press, New York, 1980.

${ }^{21}$ Yu. V. Pleskov, Yu. Ya. Gurevich, Semiconductor Photoelectrochemistry, Consultants Bureau, New York, 1986.

${ }^{22}$ A. Hamnett, in Comprehensive Chemical Kinetics, Ed. by R.G. Compton, Elsevier Science, Oxford, 1987, vol. 27, p. 61.

${ }^{23}$ J.O'M. Bockris, S.U.M. Khan, Surface Electrochemistry, Plenum Press, New York, 1993.

${ }^{24}$ N. Sato, Electrochemistry at Metals and Semiconductor Electrodes, Elsevier Science B.V., Amsterdam, 1999.

${ }^{25}$ R. Memming, Semiconductor Electrochemistry, Wiley-VCH, Weinheim, 2001.

${ }^{26}$ L. Young, Anodic Oxide Films, Academic Press, London, 1961.

${ }^{27}$ D.A. Vermilyea, in Advances in Electrochemistry and Electrochemical Engineering, Ed. by P. Delahay, Interscience Publishers, New York, 1963, Vol. 3, p. 211.

${ }^{28}$ K.E. Heusler, K.S. Yun, Electrochim. Acta, 22 (1977) 977.

${ }^{29}$ H.J. Engell, Electrochim. Acta, 22 (1977) 987.

${ }^{30}$ H. Gerischer, Corrosion Sci., 29 (1989) 257; Corrosion Sci., 31 (1990) 81.

${ }^{31}$ H. Gerischer, Electrochim. Acta, 35 (1990) 1677. 
${ }^{32}$ J.W. Schultze, M.M. Lohrengel, Electrochim. Acta, 45 (2000) 2499.

${ }^{33}$ F. Di Quarto, M. Santamaria, Corrosion Eng. Sci. Tech., 39 (2004) 71.

${ }^{34}$ F. Mott: Proc. Roy. Soc.(A), 171 (1939) 27.

${ }^{35}$ W. Shottky: Zeits. Physik, 113 (1939) 367.

${ }^{36}$ J. F. Dewald, Bell Syst. Tech. J., 39 (1960) 615; J. Phys. Chem. Solids, 14 (1960) 155.

${ }^{37}$ U. Stimming, Electrochim. Acta, 31 (1986) 415.

${ }^{38}$ L. Peter, in Comprehensive Chemical Kinetics, Ed. by R.G. Compton, Elsevier Science, Oxford, 1989, vol. 29, p. 382.

${ }^{39}$ F. Di Quarto, C. Sunseri, S. Piazza, M. Santamaria, in Handbook of Thin Films, Ed. by H.S. Nalwa, Academic Press, San Diego, CA, 2002, vol.2, p. 373.

${ }^{40}$ F. Di Quarto, M. Santamaria, C. Sunseri, in Analytical Methods in Corrosion Science and Technology, Ed. by P. Marcus, F. Mansfeld, Taylor, Francis, Boca Raton, 2005, p. 697.

${ }^{41}$ M. Pourbaix, Atlas of Electrochemical Equilibria in Aqueous Solutions, Pergamon Press, Oxford, 1966.

${ }^{42}$ K. Uosaki, H. Kita, J. Electrochem. Soc., 130 (1983) 895.

${ }^{43}$ W.H. Laflère, R.L. Van Meirhaeghe, F. Cardon, W.P. Gomes, Surface Sci., 59 (1976) 401;ibidem, 74, (1978) 125.

${ }^{44}$ W.H. Laflère, R.L. Van Meirhaeghe, F. Cardon, W.P. Gomes, J. Appl. Phys.D, 13 (1980) 2135.

${ }^{45}$ M. Tomkiewicz, J. Electrochem. Soc., 126 (1979) 1505

${ }^{46}$ G. Cooper, J. A. Turner, A.J. Nozik, J. Electrochem. Soc., 129 (1982) 1973.

${ }^{47}$ H.O. Finklea, J. Electrochem. Soc., 129 (1982) 2003.

${ }^{48}$ W.P. Gomes, F. Cardon, in Progress in Surface Science, Pergamon Press, Oxford, 1982, vol. 12, p. 155.

${ }^{49}$ H. Gerischer, R. McIntyre, J. Chem. Phys., 83 (1985) 1363.

${ }^{50}$ D.S. Ginley, M.A. Butler, in Semiconductor Electrodes, Ed. by H.O. Finklea, Elsevier Science, Oxford, 1988, p. 335.

${ }^{51}$ N. Sato, K. Kudo, T. Noda, Z. Physik. Chem. N.F., 98 (1975) 271.

${ }^{52}$ P. Marcus, V. Maurice, in Interfacial Electrochemistry, Ed. by A. Wieckowski, M. Dekker, New York, 1999, p. 541.

${ }^{53}$ U. Konig, J. W. Shultze, in Interfacial Electrochemistry, Ed. A. Wieckowski, M. Dekker, New York, 1999, p. 649.

${ }^{54}$ H. Streblow, P. Marcus in Analytical Methods in Corrosion Science and Technology, Ed. by P. Marcus, F. Mansfeld, Taylor and Francis, Boca Raton, 2005, p. 1.

${ }^{55}$ V. Maurice, P. Marcus, in Analytical Methods in Corrosion Science and Technology, Ed. by P. Marcus, F. Mansfeld, Taylor and Francis, Boca Raton, 2005, p. 133.

${ }^{56}$ C.-O.A. Olsson, D. Landolt, in Analytical Methods in Corrosion Science and Technology, Ed. by P. Marcus, F. Mansfeld, Taylor and Francis, Boca Raton, 2005, p. 733.

${ }^{57}$ S.U.M. Khan, W. Shmickler, J. Electroanal. Chem., 108 (1980) 329.

${ }^{58}$ P. Schmuki, H. Böhni, Electrochim. Acta, 40 (1995) 775.

${ }^{59}$ M. Büchler, P. Schmuki, H. Böhni, T. Stenberg, T. Mäntylä: J. Electrochem. Soc., 145 (1998) 378.

${ }^{60}$ E. Sikora, D. D. MacDonald, J. Electrochem. Soc., 147 (2000), 4087.

${ }^{61}$ V.A. Alves, C.M.A. Brett, Electrochim. Acta, 47 (2002) 2081.

${ }^{62}$ Y.M. Zeng, J. L. Luo, Electrochim. Acta, 48 (2003) 35.

${ }^{63}$ I. Diez-Perez, P. Gorostiz, F. Sanz: J. Electrochem. Soc., 150 (2003) B348.

${ }^{64}$ F. Gaben, B. Vullemin, R. Oltra, J. Electrochem. Soc., 151 (2004) B595. 
${ }^{65}$ D.G. Li, Y.R. Feng, Z.Q. Bai, J.W. Zhu, M.S. Zheng, Electrochim. Acta, 52 (2007) 7877.

${ }^{66}$ S. Fujimoto, H. Tsuchiya, Corr. Sci., 49 (2007) 195.

${ }^{67}$ J. Amri, T. Souier, B. Maliki, B. Baroux, ibidem, 50 (2008) 431.

${ }^{68}$ J.S.Blakemore, Semiconductor Statistics, Pergamon Press, Oxford (1962).

${ }^{69}$ H. Gerischer, J. Phys. Chem., 89 (1985) 4249.

${ }^{70}$ H. Gerischer, R. McIntyre, D. Scherson, W. Stork, J. Phys. Chem. Phys., 91 (1987) 1930.

${ }^{71}$ D.L. Losee, J. Appl. Phys., 46 (1975) 2204.

${ }^{72}$ G. Nogami, J. Electrochem. Soc., 129 (1982) 2219.

${ }^{73}$ E.C. Dutoit, R.L. Van Meirhage, F. Cardon, W. P. Gomes, Ber. Bunsenges. Phys. Chem., 79 (1975) 1206.

${ }^{74}$ V. Macagno, J.W. Schultze, J. Electroanal. Chem., 180 (1984) 157.

${ }^{75}$ F. Di Quarto, A. Di Paola, C. Sunseri, Electrochim. Acta, 26, (1981) 1177.

${ }^{76}$ F. Di Quarto, C. Sunseri, S. Piazza, Ber. Bunsenges. Phys. Chem., 90 (1986) 549.

${ }^{77}$ F. Di Quarto, S. Piazza, C. Sunseri, Electrochim. Acta, 35 (1990) 99.

${ }^{78}$ F. Di Quarto, V.O. Aimiuwu, S. Piazza, C. Sunseri, Electrochim. Acta, 36 (1991) 1817.

${ }^{79}$ M.H. Cohen, H. Fritzsche, S.R. Ovishinsky, Phys. Rev. Lett., 22 (1969) 1065.

${ }^{80}$ D.Adler, Amorphous Semiconductors, CRS Press, Cleveland, 1971.

${ }^{81}$ N.F. Mott, E.A. Davis, Electronic Processes in Non-crystalline Materials, $2^{\text {nd }}$ Ed., Clarendon Press, Oxford, 1979.

${ }^{82}$ J. Tauc, Amorphous and Liquid Semiconductors, Plenum Press, London, 1974.

${ }^{83}$ Amorphous Semiconductors, edited by M.H. Brodsky, Springer Verlag, Berlin, 1979.

${ }^{84}$ Physical Properties of Amorphous Materials, edited by D. Adler, B.B. Schwartz, M.C. Steele, Plenum Press, New York, 1985.

${ }^{85}$ W.E. Spear, P.G. Le Comber, A.J. Snell, Philos. Mag. B, 38 (1978) 303.

${ }^{86}$ R.A. Abram, P.J. Doherty, Philos. Mag. B, 45 (1982) 167.

${ }^{87}$ J.D. Cohen, D.V. Lang, Phys. Rev. B, 25, (1982) 5321; D.V. Lang, J.D. Cohen, J.P. Harbison, Phys. Rev. B, 25, (1982) 5285.

${ }^{88}$ I. W. Archibald, R. A. Abram, Philos. Mag. B, 48, (1983) 111.

${ }^{89}$ I. W. Archibald, R. A. Abram, Philos. Mag. B, 54, (1986) 421.

${ }^{90}$ W. E. Spear, S. H. Baker, Electrochim. Acta, 34, (1989) 1691.

${ }^{91}$ M. H. Dean, U. Stimming, J. Electroanal. Chem., 228, (1987) 135.

${ }^{92}$ M. H. Dean, U. Stimming, J. Phys. Chem., 93, (1989) 8053.

${ }^{93}$ S. Piazza, C. Sunseri, F. Di Quarto, A.I.Ch.E. Journal, 38 (1992) 219.

${ }^{94}$ F. Di Quarto, M. Santamaria, in Surface Oxide Films, V. Birss, L. Burke, A. R. Hillman, R. S. Lillard, Editors, PV 2003-25, The Electrochemical Society Proceeding Series, Pennington, NJ, 2004, p. 86.

${ }^{95}$ F. Di Quarto, F. La Mantia, M. Santamaria, Electrochim. Acta, 50, (2005) 5090.

${ }^{96}$ F. Di Quarto, F. La Mantia, M. Santamaria, Corr. Sci., 49, (2007) 186.

${ }^{97}$ F. La Mantia, M. Santamaria, F. Di Quarto, in Passivation of Metals and Semiconductors, and properties of Thin Oxide Layers, Ed. by P. Marcus, V. Maurice, Elsevier, Amsterdam, 2006, p. 343.

${ }^{98}$ C. da Fonseca, M. G. Ferreira, M. da Cunha Belo, Electrochim. Acta, 39, (1994) 2197.

${ }^{99}$ E. Becquerel, C. R. Hebd. Séan. Acad. Sci., 9 (1839) 561.

${ }^{100}$ A.W. Copeland, O.D. Black, A.B. Garrett, Chem. Rev., 31 (1942) 177. 
${ }^{101}$ E. K. Oshe, I. L. Rozenfel'd, Elektrokhimiya, 4 (1968) 1200.

${ }^{102}$ R. Williams: J. Chem. Phys., 32 (1960) 1505.

${ }^{103}$ W. Paatsch: J. Phys., 38 (1977) C5-151

${ }^{104}$ E. Angelini, M. Maja, P. Spinelli: J. Phys., 38 (1977) C5-261.

${ }^{105}$ S. M. Wilhelm, Y. Anizawa, Chaung-Yi Liu, N. Hackermann, Corros. Sci., 22 (1982) 791.

${ }^{106}$ A. Fujishima, K. Honda, Nature, 238 (1972) 37.

${ }^{107}$ M. A. Butler, J. Appl. Phys., 48 (1977) 1914.

${ }^{108}$ Faraday Discussion No.70, The Royal Society of Chemistry, London, 1980.

${ }^{109}$ Photoeffects at Semiconductor-Electrolyte Interfaces, ACS Symposium Series 146 Ed. A. J. Nozik, American Chemical Society, Washington D.C., 1981.

${ }^{110}$ F. Di Quarto, G. Russo, C. Sunseri, A. Di Paola: J. Chem. Soc. Faraday Trans.1, 78 (1982) 3433

${ }_{111}^{11}$ R.H. Wilson. J. Appl. Phys., 48 (1977) 4292.

${ }^{112}$ H. Reiss. J. Electrochem. Soc., 125 (1978) 937.

${ }^{113}$ W.J. Albery, P.N. Bartlett, A. Hamnett, M.P. Dare-Edwards. J. Electrochem. Soc., 128 (1981) 1492

${ }^{114}$ F. El Guibaly, K. Colbow. J. Appl. Phys., 53 (1982) 1737.

${ }^{115}$ W.W. Gärtner. Phys. Rev., 116 (1959) 84.

${ }^{116}$ J. Reichman, Appl. Phys. Lett., 36 (1980) 574.

${ }^{117}$ R.U. E’t Lam, D.R. Franceschetti, Mater. Res. Bull., 17 (1982) 1081.

${ }^{118}$ F. Di Quarto, A. Di Paola, S. Piazza, C. Sunseri, Solar Energy Mater., 11 (1985) 419.

${ }^{119}$ S.U.M. Khan, J.O'M. Bockris J. Appl. Phys., 52 (1981) 7270.

${ }^{120}$ D.M. Pai, R.C. Enck. Phys. Rev. B, 11 (1975) 5163.

${ }^{121}$ F. Di Quarto, S. Piazza, C. Sunseri. Electrochim. Acta, 38 (1993) 29.

${ }^{122}$ M. Santamaria, F. Di Quarto, H. Habazaki, Corr. Sci., 50 (2008) 2012.

${ }^{123}$ F. Di Quarto, S. Piazza, R. D'Agostino, C. Sunseri. J. Electroanal. Chem., 228 (1987) 119.

${ }^{124}$ F. Di Quarto, S. Piazza, C. Sunseri. J. Chem. Soc., Faraday Trans. 1, 85 (1989) 3309.

${ }^{125}$ S. Piazza, C. Sunseri, F. Di Quarto. J. Electroanal. Chem., 293 (1990) 69.

${ }^{126}$ N.F. Mott, E.A. Davis, Electronic Processes in Non-crystalline Materials, $2^{\text {nd }}$ Ed., Clarendon Press, Oxford, 1979, p.275.

${ }^{127}$ D.L. Wood, J. Tauc, Phys. Review B, 5 (1972) 3144.

${ }^{128}$ Di Quarto, S. Piazza, A. Splendore, C. Sunseri. "Oxide Films on Metals and Alloys", p. 311, B.R. McDougall, R.S. Alwitt, T.A. Ramanarayanan, Editors, PV 92-22, The Electrochemical Society Proceeding Series, Pennington, NJ, 1992

${ }^{129}$ T. Watanabe, H. Gerischer. J. Electroanal. Chem., 122 (1981) 73.

${ }^{130}$ Di Quarto, G. Tuccio, A. Di Paola, S. Piazza, C. Sunseri, "Oxide Films on Metals and Alloys" p. 25, K. Hebert, G.E. Thompson, Editors, PV 94-25, The Electrochemical Society Proceeding Series, Pennington, NJ, 1994.

${ }^{131}$ C. Sunseri, S. Piazza, F. Di Quarto, Mater. Sci. Forum, 185-188 (1995) 435.

${ }^{132}$ F. Di Quarto, S. Piazza, C. Sunseri, M. Yang, S.M. Cai, Electrochim. Acta, 41 (1996) 2511.

${ }^{133}$ S. Piazza, A. Splendore, A. Di Paola, C. Sunseri, F. Di Quarto, J. Electrochem. Soc., 140 (1993) 3146

${ }^{134}$ Yu.Ya. Gurevich, Yu. V. Pleskov, Z.A. Rotenberg, Photoelectrochemistry, Plenum Press, New York, 1980. 
${ }^{135}$ M. Santamaria, F. Di Quarto, S. Zanna, P. Marcus, Electrochim. Acta, 53 (2007) 1314.

${ }^{136}$ P. Han, D.M. Bartels, J. Phys. Chem., 94 (1990) 5824.

${ }^{137}$ F. Di Quarto, C. Sunseri, S. Piazza, M.C. Romano. J. Phys. Chem. B, 101 (1997) 2519.

${ }^{138}$ J.C. Phillips, Bonds and Bands in Semiconductors, Academic Press, New York, 1973.

${ }^{139}$ L. Pauling, The Nature of Chemical Bond, chapter 3, Cornell University Press, Ithaca, NY, 1960.

${ }^{140}$ W. Gordy, W.J.O. Thomas., J. Phys. Chem., 24 (1956) 439.

${ }^{141}$ L. Allred, J. Inorg. Nucl. Chem., 17 (1961) 215.

${ }^{142}$ W. Teng, J. F. Muth, U. Ozgur, M. J. Bergman, H. O. Everitt, A. K. Sharma, C. Jin, J. Narayan. Appl. Phys. Letters, 76 (2000) 979.

${ }^{143}$ M. Santamaria, F. Di Quarto, P. Skeldon, G.E. Thompson, J. Electrochem. Soc., 153 (2006) B518.

${ }^{144}$ M. Santamaria, D. Huerta, S. Piazza, C. Sunseri, F. Di Quarto. J. Electrochem. Soc., 147 (2000) 1366

${ }^{145}$ H. Kim, N. Hara, K. Sugimoto, . J. Electrochem. Soc., 146 (1999) 955.

${ }^{146}$ M. Santamaria, F. Di Quarto, H. Habazaki, Electrochim. Acta, 53 (2008) 2272.

${ }^{147}$ F. Di Quarto, M. Santamaria, P. Skeldon, G.E. Thompson, Electrochim. Acta, 48 (2003) 1143

${ }^{148}$ A. R. Newmark, U. Stimming, Langmuir, 3 (1987) 905.

${ }^{149}$ F. Di Quarto, M.C. Romano, M. Santamaria, S. Piazza, C. Sunseri, Russian Journal of Electrochemistry, 36 (2000) 1203.

${ }^{150}$ M. Santamaria, E. Adragna, F. Di Quarto, Electrochem. and Solid State Letters, 8 (2005) B12.

${ }^{151}$ U. Stimming, Electrochim. Acta, 3 (1986) 415.

${ }^{152}$ M. K. Carpenter, D.A. Corrigan, J. Electrochem. Soc., 136 (1989)1022.

${ }^{153}$ L. Anicai, R. Masi, M. Santamaria, F. Di Quarto, Corros. Sci, 47 (2005) 2883.

${ }^{154}$ F. Criminisi. Chem. Eng. Degree Thesis, Università di Palermo, April, 2002

${ }^{155}$ Di Quarto, M. Santamaria, N. Mallandrino, V. Laget, R. Buchheit, K. Shimizu, J. Electrochem. Soc., 150 (1989) B462.

${ }^{156}$ F. Di Quarto, S. Piazza, C. Sunseri, Current Topics in Electrochemistry, Vol. 3, p. 357, edited by J.C. Alexander, Council of Scientific Research Integration, Trivarandum, 1994

${ }^{157}$ F. Di Quarto, S. Piazza, C. Sunseri, Mater. Sci. Forum, 192-194 (1995) 633.

${ }^{158}$ L. Anicai, S. Piazza, M. Santamaria, C. Sunseri, F. Di Quarto, ATB Metallurgie, (2000) 175.

${ }^{159}$ F. Di Quarto, S. Piazza, C. Sunseri, Corros. Sci., 31 (1990) 721.

${ }^{160}$ H.Tsuchiya, S. Fujimoto, O. Chihara, T. Shibata, Electrochim. Acta, 47 (2002) 4357.

${ }^{161}$ H.Tsuchiya, S. Fujimoto,T. Shibata, J. Electrochem. Soc., 151 (2004) B39.

${ }^{162}$ S. Fujimoto, H.Tsuchiya, Corr. Science, 49 (2007) 195.

${ }^{163}$ T.L. Sudesh, L. Wijesinghe, D. J. Blackwood, J. of Physics: Conference Series, 28 (2006) 74.

${ }^{164}$ T.L. Sudesh, L. Wijesinghe, D. J. Blackwood, Appl. Surf. Sci., 253 (2006) 1006.

${ }^{165}$ T.L. Sudesh, S.L. Wijesinghe, D. J. Blackwood, J. Electrochem. Soc., 154 (2007) C16.

${ }^{166}$ T.L. Sudesh, L. Wijesinghe, D. J. Blackwood, Corros. Sci., 50 (2008) 23. 
${ }^{167}$ S Virtanen, P Schmuki, H. Bohni, P. Vuoristo, T. Mantyla, J. Electrochem. Soc., 142 (1995) 3067.

${ }^{168}$ P. Schmuki, M. Buchler, S Virtanen, H Bohni, R. Muller, L.J. Gauchler, J. Electrochem. Soc., 142 (1995) 3336.

${ }^{169}$ M. Buchler, P. Schmuki, H Bohni, T. Stenberg, T. Mantyla, J. Electrochem. Soc., 145 (1998) 378.

${ }^{170}$ A.J. Davenport, L.J. Oblonsky, M.P. Ryan, M.F. Toney, J.Electrochem. Soc., 147 (2000) 2162.

${ }^{171}$ S. Piazza, M. Sperandeo, C. Sunseri, F. Di Quarto, Corros. Sci., 46 (2004) 831.

${ }^{172}$ C. Sunseri, S. Piazza, A. Di Paola, F. Di Quarto, J. Electrochem. Soc., 134 (1987) 2410.

${ }^{173}$ G. Dagan, W-M. Shen, M. Tomkiewicz, J. Electrochem. Soc., 139 (1992) 1855.

${ }_{174}$ A. Di Paola, F. Di Quarto, C. Sunseri, Corros. Sci., 26 (1986) 935.

${ }^{175}$ T. D. Burleigh, R.M. Latanision. J. Electrochem. Soc., 134 (1987) 137.

${ }^{176}$ A. Di Paola, D. Shukla, U. Stimming, Electrochim. Acta, 36 (1991) 345.

${ }^{177}$ M.J. Kloppers, F. Bellucci, R.M. Latanision, Corrosion, 48 (1992) 229.

${ }^{178}$ P. Schmuki, H. Bohni, J. Electrochem. Soc., 139 (1992) 1908.

${ }^{179}$ P. Schmuki, H. Bohni, F. Mansfeld. J. Electrochem. Soc., 140 (1993) L119.

${ }^{180}$ A.M.P. Simoes, M.G.S. Ferreira, B. Rondot, M. Da Cunha Belo, J. Electrochem. Soc., 137 (1990) 82.

${ }^{181}$ N.E. Hakiki, M. Da Cunha Belo, A.M. P. Simoes, M.G.S. Ferreira, J. Electrochem. Soc., 145 (1998) 3821.

${ }^{182}$ N.E. Hakiki, M. Da Cunha Belo, M.G.S. Ferreira, Electrochim. Acta, 44 (1999) 2473.

${ }^{183}$ M.F. Montemor, M.G.S. Ferreira, N.E. Hakiki, M. Da Cunha Belo, Corros. Sci., 42 (2000) 1635

${ }^{184}$ L.J. Oblonsky, M.P. Ryan, H.S. Isaacs, J. Electrochem. Soc., 145 (1998) 1922.

${ }^{185}$ P. Marcus, V. Maurice, Interfacial Electrochemistry, Ed. A. Wieckowski, M. Dekker Inc., NY, 1999, p. 541.

${ }^{186}$ D. Hamm, C-O. A. Olsson, D. Landolt, Corros. Sci., 44 (2002) 1009.

${ }^{187}$ Keller, H-H. Streblow, Corros. Sci., 46 (2004) 1939. 